LBL -31124

DE92 014506

\title{
Low-Frequency Nuclear Quadrupole Resonance with a dc SQUID
}

\author{
Jih-Wen Chang \\ Department of Chemistry \\ University of California \\ and \\ Materials Sciences Division \\ Lawrence Berkeley Laboratory \\ University of California \\ Berkeley, California 94720
}

July 1991

This work was supported by the Director, Office of Energy Research, Office of Basic Energy Sciences, Materials Sciences Division, of the U.S. Department of Energy under Contract No. DE-AC03-76SF00098. 


\title{
Low-Frequency Nuclear Quadrupole Resonance \\ with a dc SQUID
}

by

\author{
Jih-Wen Chang
}

\begin{abstract}
Conventional pure nuclear quadrupole resonance (NQR) is a technique well suited for the study of very large quadrupolar interactions. Numerous nuclear magnetic resonance (NMR) techniques have been developed for the study of smaller quadrupolar interactions. However, there are many nuclei which have quadrupolar interactions of intermediate strength. Quadrupolar interactions in this region have traditionally been difficult or unfeasible to detect. This work describes the development and application of a SQUID NQR technique which is capable of measuring intermediate strength quadrupolar interactions, in the range of a few hundred kilohertz to several megahertz. In this technique, a dc SQUID (Superconducting QUantum Interference Device) is used to monitor the longitudinal sample magnetization, as opposed to the transverse magnetization, as a rf field is swept in frequency. This allows the detection of low-frequency nuclear quadrupole resonances over a very wide frequency range with high sensitivity. The theory of this NQR technique is discussed and a description of the dc SQUID system is given. In the following
\end{abstract}


chapters, the spectrometer is discussed along with its application to the study of samples containing half-odd-integer spin quadrupolar nuclei, in particular boron-11 and aluminum-27. The feasiblity of applying this NQR technique in the study of samples contaizing integer spin nuclei is discussed in the last chapter. 


\section{ACKNOWLEDGEMENTS}

I would like to thank Professor Alexander Pines for his continuous encouragement and support during my graduate studies. Chuck Connor has been a driving force in the development and success of this research project since the beginning, and it has been a pleasure working with him. The help of Dr. Hans Huber and Dr. Gerry Chingas, in the initial phases of this project, is gratefully acknowledged. The assistance of Bruce Black in some closing experiments was appreciated.

The educational discussions on zero-field NMR and NQR with Tony Bielecki, Ann Thayer, and Tom Jarvie were much appreciated, as were the helpful discussions with David Shykind, Malaine Trecoske, Karl Mueller, and Steve Rucker. I wish to thank Steve Rucker and Karl Mueller for their help in producing this $\operatorname{IAT}_{\mathrm{E}} \mathrm{X}$ document. Finally, special thanks to my family for all of their encouragement and support throughout my studies. 


\section{Contents}

1 Introduction 1

2 Theory of Z-axis CW NQR 5

2.1 Hamiltonians .................... 5

2.2 Theory of Z-axis NQR . . . . . . . . . . . . . 8

2.2.1 Single Crystal Sample . . . . . . . . . . . . . 8

2.2.2 Adaptations for Powder Sarraples . . . . . . . . . . 20

2.2.3 Miscellaneous Considerations ... . . . . . . . . . 26

2.3 NQR Resonance Frequencies . . . . . . . . . . . . . . . . . . . 29

3 General Principles of the de SQUID System 31

3.1 Introduction ..................... 31

3.2 Equations Describing the dc SQUID . . . . . . . . . . . 34

3.3 Coupling Magnetic Flux to the SQUID . . . . . . . . . . . 40

3.4 Output Voltage of the SQUID System . . . . . . . . . . . 45

4 SQUID NQR Spectrometer

4.1 Probe and Spectrometer Design Considerations . . . . . . . . 53

4.2 Overview of the Spectrometer ............... 60 
4.3 Low-Temperature Probe . . . . . . . . . . . . . . . 63

4.4 Cryostat and Room Temperature Shielding . . . . . . . . . . . 67

4.5 rf Sources and Data Acquisition . . . . . . . . . . . . . 69

4.6 Spectrometer Performance . . . . . . . . . . . . . . 72

$5 \quad$ I $=\frac{3}{2}$ NQR Results $\quad 79$

5.1 Introduction . . . . . . . . . . . . . 79

5.2 Boron Nitride, BN . . . . . . . . . . . . . . 81

5.3 Boron Hydrates, $\mathrm{B}_{2} \mathrm{O}_{3} \cdot \mathrm{xH}_{2} \mathrm{O}(\mathrm{x}=3,1$, and 0$) \ldots . \ldots 86$

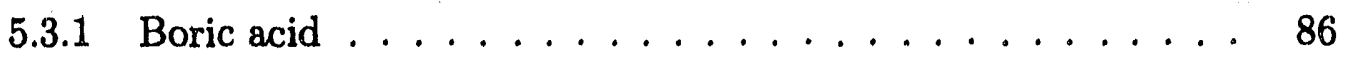

5.3 .2 Boron monohydrate . . . . . . . . . . . . 89

5.3.3 Boron oxide (crystalline) . . . . . . . . . . . . 89

5.3.4 Further boron hydrates . . . . . . . . . . . . 91

5.4 Boron Oxide Glass, $\mathrm{B}_{2} \mathrm{O}_{3} \ldots \ldots \ldots \ldots$

$6 \quad I=\frac{5}{2}$ NQR Results 101

6.1 Introduction . . . . . . . . . . . . . . . 101

6.2 Sapphire, $\alpha-\mathrm{Al}_{2} \mathrm{O}_{3} \ldots \ldots \ldots \ldots$. . . . . . . . . . . . 102

6.2.1 Single Crystal Sample . . . . . . . . . . . . 102

6.2 .2 Powder Sample ................... 116

6.3 Petalite, $\mathrm{LiAlSi}_{4} \mathrm{O}_{10} \ldots \ldots \ldots \ldots$

6.4 Further SQUID NQR Experiments Possible . . . . . . . . . . . 122

$7 \mathrm{I}=1$ NQR Theory and Results $\quad 124$

7.1 Introduction . . . . . . . . . . . . . . . . . 124

7.2 Theory for $I=1$ Nuclei . . . . . . . . . . . . . . 125 
7.3 Solid Nitrogen, $\alpha-\mathrm{N}_{2} \ldots \ldots \ldots$

A Analytical Solution to the Zero-Field Energy Levels of a Spin $I=\frac{5}{2}$

Nucleus

133 


\section{List of Figures}

2.1 Energy levels of a $\operatorname{spin} I=\frac{3}{2}$ nucleus $\ldots \ldots \ldots \ldots$

2.2 Energy levels of a $\operatorname{spin} I=\frac{5}{2}$ nucleus $\ldots \ldots \ldots \ldots \ldots$

2.3 Theoretical signal from a single crystal sample, ignoring relaxation . 13

2.4 Cross-relaxation processes between $+m$ and $-m$ manifolds $\ldots \ldots 16$

2.5 Simulated lineshapes of a single crystal sample . . . . . . . . 18

2.6 Simulated lineshapes of a powder sample $\ldots \ldots \ldots \ldots$

3.1 Schematic of a dc SQUID $\ldots \ldots \ldots \ldots \ldots \ldots \ldots$

3.2 Schematic of a Josephson junction . . . . . . . . . . . . . 36

3.3 Voltage versus flux curves of the de SQUID . . . . . . . . . . . 39

3.4 Bias current as a function of the voltage across the dc SQUID and magnetic flux through the SQUID . . . . . . . . . . . . 41

3.5 Transformer used to transfer flux from the sample to the SQUID . . 43

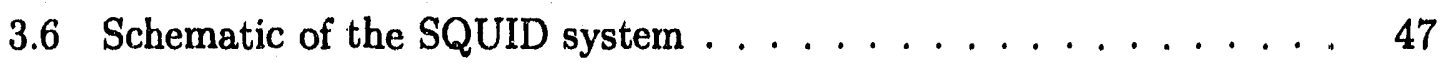

3.7 Lock-in detection scheme of the SQUID system $\ldots \ldots \ldots \ldots$

3.8 Final output voltage versus flux curve of the SQUID system $\ldots . .50$

4.1 Schematic of the low-temperature low-pass filter (shunt resistor ar-

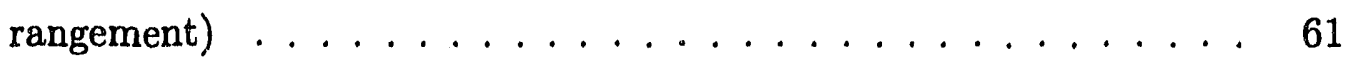


4.2 Schematic of the SQUID spectrometer . . . . . . . . . . 62

4.3 Cross-section of the low-temperature probe . . . . . . . . . . . 64

4.4 Schematic of the sample tube and sample stick . . . . . . . . . 68

4.5 Schematic of the room-temperature low-pass filter . . . . . . . . 71

4.6 Noise spectrum of the SQUID system . . . . . . . . . . 73

4.7 rf attenuation provided by the shunt resistance . . . . . . . . . . 74

4.8 Response of the SQUID after a strong magnetic field pulse . . . . . 78

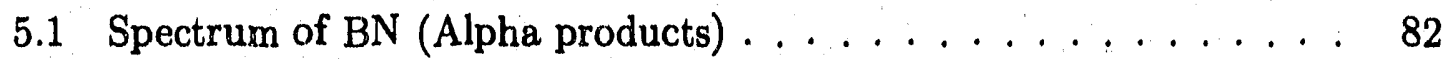

5.2 Spectrum of BN (ESPI powder) . . . . . . . . . . 84

5.3 High-resolution spectrum of BN (ESPI hot-pressed rod) . . . . . . . 85

5.4 Structure of boric acid $\left(\mathrm{B}_{2} \mathrm{O}_{3} \cdot 3 \mathrm{H}_{2} \mathrm{O}\right) \ldots \ldots \ldots$

5.5 Spectra of borates $\left(\mathrm{B}_{2} \mathrm{O}_{3} \cdot \mathrm{xH}_{2} \mathrm{O}\right.$ where $\mathrm{x}=3$, 1, and 0 ) (natural abundance in boron) ....................... 88

5.6 Spectra of ${ }^{11}$ B-enriched borates ............... 92

5.7 Spectrum of $\mathrm{B}_{2} \mathrm{O}_{3}$ glass . . . . . . . . . . . . . . . 99

6.1 Structure of sapphire $\left(\alpha-\mathrm{Al}_{2} \mathrm{O}_{3}\right) \ldots \ldots 103$

6.2 Spectrum of $\alpha-\mathrm{Al}_{2} \mathrm{O}_{3}$ single crystal . . . . . . . . . . 105

6.3 Complete spectrum of $\alpha-\mathrm{Al}_{2} \mathrm{O}_{3}$ single crystal using a forward sweep 107

6.4 Forward sweeps of $\alpha-\mathrm{Al}_{2} \mathrm{O}_{3}$ single crystal using various sweep rates 108

6.5 Simulated and experimental sweeps of $\alpha-\mathrm{Al}_{2} \mathrm{O}_{3}$ single crystal . . . 109

6.6 Sweeps of $\alpha-\mathrm{Al}_{2} \mathrm{O}_{3}$ single crystal using circularly polarized rf . . . . 111

6.7 "Absorption" spectrum of $\pm \frac{3}{2} \leftrightarrow \pm \frac{5}{2}$ resonance of $\alpha-\mathrm{Al}_{2} \mathrm{O}_{3}$ single

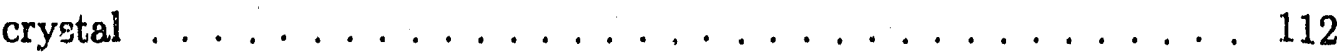

6.8 Signal height versus of strength for the $\alpha-\mathrm{Al}_{2} \mathrm{O}_{3}$ single crystal . . . . 114 
6.9 Signal height versus sweep rate for the $\alpha-\mathrm{Al}_{2} \mathrm{O}_{3}$ single crystal . . . . 115

6.10 Spectrum of $\alpha-\mathrm{Al}_{2} \mathrm{O}_{3}$ powder . . . . . . . . . . . 117

6.11 Forward sweeps of $\alpha-\mathrm{Al}_{2} \mathrm{O}_{3}$ powder using various sweep rates . . . 118

6.12 Simulated and experimental sweeps of $\alpha-\mathrm{Al}_{2} \mathrm{O}_{3}$ powder $\ldots \ldots$

6.13 Spectrum of petalite $\left(\mathrm{LiAlSi}_{4} \mathrm{O}_{10}\right) \ldots \ldots \ldots \ldots$

7.1 Energy levels of a spin $I=1$ nucleus $\ldots \ldots \ldots \ldots$

7.2 Calculated signal height versus $z$ factor $\left(z=\frac{\gamma B_{0}}{\left(\frac{e^{2} q Q}{d h}\right) \eta}\right)$ for spin $I=1$

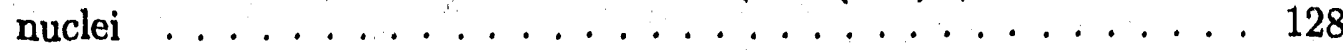

7:3 Calculated signal height versus $\eta$ for spin $I=1$ nuclei $\ldots \ldots \ldots$

7.4 Spectrum of $\alpha-\mathrm{N}_{2} \ldots \ldots \ldots \ldots \ldots \ldots$ 


\section{List of Tables}

2.1 Calculated z-magnetizations for samples with spin $I=\frac{3}{2}$ and $I=\frac{5}{2}$

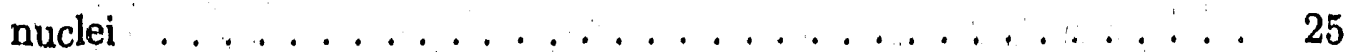

4.1 Calculated and measured sample magnetizations . . . . . . . 77

5.1 Physical data on ${ }^{10} \mathrm{~B},{ }^{11} \mathrm{~B}$, and ${ }^{27} \mathrm{Al}$ nuclei $\ldots \ldots . \ldots 80$

5.2 Measured characteristics of the ${ }^{11} \mathrm{~B}$ resonances in the three polycrystalline borates, $\mathrm{B}_{2} \mathrm{O}_{3} \cdot \mathrm{xH}_{2} \mathrm{O}(\mathrm{x}=3,1$, and 0$) \ldots \ldots 90$

5.3 Calculated quadrupolar parameters for the three borates, $\mathrm{B}_{2} \mathrm{O}_{3} \cdot \times \mathrm{H}_{2} \mathrm{O}$ $(x=3,1$, and 0$) \ldots \ldots \ldots \ldots$

5.4 Calculated quadrupolar parameters for $\mathrm{B}_{2} \mathrm{O}_{3} \cdot 3 \mathrm{H}_{2} \mathrm{O}$ from the work of Butler and Brown ................... 97 


\section{Chapter 1}

\section{Introduction}

Nuclear Quadrupole Resonance (NQR) involves the study of an electrostatic interaction between quadrupolar nuclei (spin $I>\frac{1}{2}$ ) and the electric field gradients surrounding it [1]. Since these electric field gradients are produced primarily by the electrons and other atoms immediately around the quadrupolar nuclei, NQR is a powerful tool in the structure determination of solids $[2,3,4]$. Pure NQR is traditionally carried out in zero magnetic field where the quadrupolar interaction is much stronger than other existing interactions such as dipolar interactiors. This allows for the easy and accurate determination of the resonance frequencies when they are large, i.e. larger than about $5 \mathrm{MHz}$. In a typical NQR experiment, the sample is irradiated with radiofrequency (rf) energy while it rests in a coil tuned to the resonance frequency $[5,6]$. When the $\mathrm{rf}$ is of the proper frequency and excites transitions between the spin levels, the magnetization of the sample oscillates at the resonance frequency. This oscillating transverse magnetization within the coil produces a potential across the coil, according to the Faraday effect. The voltage across the coil is then amplified and recorded. In a continuous wave (CW) exper- 
iment, the recorded signal is the spectrum. In the case of a pulsed experiment, the recorded signal must be Fourier transformed to yield the spectrum. For either technique. the sensitivity of the Faraday effect is proportional to the rate of change in magnetization $\left(V \propto \frac{d \Phi}{d t}\right)[7]$, meaning the potential across the coil decreases as the resonance frequency decreases. This imposes a lower limit on the resonance frequency which conventional NQR is capable of detecting in a reasonable amount of experimental time. Previously, this limit has been placed at several megahertz. Recent advances have made it possible to extend this limit down to several hundred kilohertz, however with long acquisition times [8].

Nuclear Magnetic Resonance (NMR) has also been used to study quadrupolar interactions $[9,10]$. Since NMR is usually conducted at very high magnetic fields, the quadrupolar interaction appears only as a perturbation on the Zeeman energy levels. Techniques similar to those used in pure NQR are used to observe the resonances whose frequency is determined mainly by the strength of the strong magnetic field. Under normal circumstances, one is able to observe the so-called central transition $\left(-\frac{1}{2} \leftrightarrow+\frac{1}{2}\right)$ only due to the anisotropy of space imposed by the strong magnetic field. Most solid samples are in a powdered form making the central transition broad and with distinctive features, called powder patterns $[11,12]$. The experimentalist is left with the task of extracting information about the quadrupolar interaction from the feacures of the powder pattern and simulating the spectra. The breadth of the powder pattern is proportional to the strength of the quadrupolar interaction, thereby limiting NMR to the study of quadrupolar interactions of relatively weak strength. The situation becomes even more complicated when more than one distinct site exists within the sample, resulting in overlapping powder 
patterns. However, recent advances in techniques have made the use of conventional $[13,14,15,16]$ and field-cycling $[17,18]$ NMR very attractive for the study of quadrupolar nuclei.

Many important nuclei, such as ${ }^{11} \mathrm{~B},{ }^{14} \mathrm{~N},{ }^{23} \mathrm{Na}$, and ${ }^{27} \mathrm{Al}$, are found in technologically important solids and participate in intermediate strength quadrupolar interaitions. The objective of this dissertation is to present a technique which can be used to study quadrupolar interactions of intermediate strength, where the pure NQR resonances appear in the region of several hundred kilohertz to several megahertz. It seems almost impossible to remove the broadening of the resonance imposed by the strong magnetic field in NMR, so one is left with the option of working in zero or very low magnetic fields. This neccesitates the use of an unconventional detector, called the SQUID (Superconducting QUantum Interference Device), to avoid the low-frequency limitations of the Faraday detector. The SQUID [19, 20, 21], unlike the Faraday detector which indirectly measures the oscillating magnetic flux as voltage, directly measures magnetic flux itself $(V \propto \Phi)$. Its operating principles are such that its sensitivity does not depend on how fast the magnetic flux is changing [22]. This allows one, with the use of a SQUID, to extend the previous frequency limitations of pure NQR even lower and investigate those quadrupolar interactions of intormediate strength. This is accomplished by sweeping an irradiating rf field, in frequency, while applying a small de magnetic field to the sample. When the rf is on resonance, it will induce a large change in the z-magnetization of the sample, magnetization along the same direction as the dc field. The z-magnetization of the sample is monitored by a dc SQUID as the rf sweeps in frequency, resulting in the spectrum. It is hoped that this SQUID NQR technique will find wide-spread 
application in the study of the structures of solids.

Chapter II presents the theory needed to understand the principles of z-axis NQR, a technique well suited for use with the SQUID. The theory for a single crystal sample and a powder sample is shown along with miscellaneous extensions and a discussion on NQR resonance frequencies for $\operatorname{spin} I=\frac{3}{2}$ and $I=\frac{5}{2}$ nuclei. The operating principles of the dc SQUID system and the techniques used to transfer magnetic flux from the sampla to the SQUID are explained in Chapter III. Chapter IV discusses design considerations, a description of the spectrometer into which the SQUID system was incorporated, and examines the performance of the spectrometer. The NQR results for some compounds containing spin $I=\frac{3}{2}\left({ }^{11} \mathrm{~B}\right)$ and $I=\frac{5}{2}$ $\left({ }^{27} \mathrm{Al}\right)$ nuclei, along with a discussion on the results, are shown in Chapters $\mathrm{V}$ and VI. The last chapter is used to show a result for ${ }^{14} \mathrm{~N}(I=1$ '. along with a discussion on the possibility of using this technique to observe quadrupole resonances from nuclei with integer spin, in particular spin $I=1$. Appendix A presents the derivation of the analytical solution to the energy levels of a spin $I=\frac{5}{2}$ nucleus in zero magnetic field. 


\section{Chapter 2}

\section{Theory of Z-axis CW NQR}

\subsection{Hamiltonians}

The main interaction that this thesis is concerned with is the quadrupolar interaction which describes the electrostatic interaction between the quadrupolar nucleus and the electric field gradients it feels [5]. Quadrupolar nuclei are non-spherically symmetric in shape and always have a spin $(I)$ greater than or equal to 1 . The electric field gradients felt by these nuclei are produced by the surrounding electrons and nuclei. Thus, studying this quadrupolar interaction is an excellent method to determine the local environment of the quadrupolar nucleus and the bonding in which it participates $[1,2,3]$. The quadrupolar Hamiltonian, in its principal axis system (PAS), may be written as

$$
H_{Q}=\frac{e^{2} q Q}{4 I(2 I-1)}\left[3 I_{z}^{2}-I^{2}+\eta\left(I_{x}^{2}-I_{y}^{2}\right)\right]
$$

where $e^{2} q Q$ is the quadrupole coupling constant (QCC) and $\eta$ is the asymmetry parameter. The quadrupole coupling constant is a product of two quantities, the magnitude of the electric field gradient along the z-axis of the principal axis system 
$(e q)$ and the electric quadrupole moment of the nucleus $(e Q)$, giving us a measure of the strength of the interaction. The principal axis system (PAS) of this interaction is defined such that the $\mathrm{z}$-axis is along the direction where the electric field gradient is largest in absolute magnitude and the $\mathrm{x}$-axis is along the direction where the magnitude electric field gradient is the smallest. In other words, $\left|V_{z z}\right| \geq\left|V_{y y}\right| \geq\left|V_{x x}\right|$ and all other components of the electric field gradient tensor are zero in the principal axis system. The asymmetry parameter $(\eta)$ is defined as

$$
\eta=\frac{V_{x x}-V_{y y}}{V_{z z}}
$$

and is a measure of the deviation from cylindrical symmetry of the interaction around the z-axis. The magnitude orderinm of the electric field gradients as given above, along with the restriction imposed by Laplace's equation $\left(V_{x x}+V_{y y}+V_{z z}=0\right.$, where $V_{i j}=0$ when $i \neq j$ ), restricts $\eta$ to the range between 0 and 1 . An asymmetry parameter of 0 indicates an electric field gradient which is axially symmetric about the $z$-axis while a value of 1 indicates a severe deviation from cylindrical symmetry. It is of importance to note that true cylindrical symmetry about the z-axis is not necessary to achieve an asymmetry parameter of 0 [9]. It has been shown that 3-fold symmetry about the z-axis is sufficient to obtain an asymmetry parameter of 0 .

The second interaction of concern is the Zeeman interaction which describes the magnetic interaction between the spin of the nucleus with the externally applied magnetic field. The Zeeman Hamiltonian is

$$
H_{M}=-\gamma \hbar I_{Z} B_{0}
$$

where $\gamma$ is the gyromagnetic ratio of the nucleus and $I_{Z}$ is the component of spin in 
the same direction as the applied magnetic field $B_{0}$. When the Zeeman interaction is much weaker than the quadrupolar interaction, as is true in this work, the Zeeman interaction must be rewritten in the axis system of the quadrupolar interaction as

$$
H_{M}=-\gamma \hbar B_{0}\left[I_{z} \cos \theta+I_{x} \sin \theta \cos \phi+I_{y} \sin \theta \sin \phi\right]
$$

The angle $\theta$ and $\phi$ are the polar and azimuthal angles, respectively, which relate the magnetic field axis to the principal axis system of the quadrupolar interaction.

The third interaction is between the spins and the radiofrequency (rf) waves used to excite transitions between the various spin levels. The Hamiltonian for linearly polarized rf along the $\mathrm{x}$-axis of the principal axis system is

$$
H_{1, l_{p}}=-\gamma \hbar B_{1} I_{x} \cos (\omega t)
$$

while if the rf is circularly polarized

$$
H_{1, c p}=-\gamma \hbar B_{1}\left[I_{x} \cos (\omega t) \pm I_{y} \sin (\omega t)\right]
$$

where the sign before the $I_{y}$ term determines the direction (left or right) of circular polarization.

The last interaction of concern is the dipolar interaction between two magnetic dipoles (spins) [10] and when expressed in the principal axis system of the dipolar interaction is

$$
H_{D}=\frac{\gamma_{1} \gamma_{2} \hbar^{2}}{r^{3}}(A+B+C+D+E+F)
$$

where

$$
A=\left(1-3 \cos ^{2} \theta\right) I_{1 z} I_{2 z},
$$




$$
\begin{aligned}
& B=-\frac{1}{4}\left(1-3 \cos ^{2} \theta\right)\left(I_{1}^{+} I_{2}^{-}+I_{1}^{-} I_{2}^{+}\right) \\
& C=-\frac{3}{2} \sin \theta \cos \theta e^{-i \phi}\left(I_{1}^{+} I_{2 z}+I_{1 z} I_{2}^{+}\right) \\
& D=-\frac{3}{2} \sin \theta \cos \theta e^{-i \phi}\left(I_{1}^{-} I_{2 z}+I_{1 z} I_{2}^{-}\right) \\
& E=-\frac{3}{4} \sin ^{2} \theta e^{-2 i \phi}\left(I_{1}^{+} I_{2}^{+}\right), \\
& F=-\frac{3}{4} \sin ^{2} \theta e^{2 i \phi}\left(I_{1}^{-} I_{2}^{-}\right) .
\end{aligned}
$$

The z-axis of the dipolar interaction is defined by the internuclear vector between the two spins and $r$ is the internuclear distance. The angles $\theta$ and $\phi$ relate the dipolar interaction axis system to the axis system of the applied magnetic field. If the nucleus of interest is dipolar coupled to more than one spin, then the overall dipolar interaction is modified to be the sum of the various individual dipolar interactions.

\subsection{Theory of Z-axis NQR}

\subsubsection{Single Crystal Sample}

In this section, we look at the example of a spin $I=\frac{3}{2}$ nucleus in a single crystal sample where the weak magnetic field is applied along the z-axis of an axially symmetric quadrupolar principal axis system $[5,23]$. The energy levels for such a system have been worked out and are given by

$$
\begin{aligned}
& E_{ \pm \frac{3}{2}}=\frac{e^{2} q Q}{4} \mp \frac{3}{2} \gamma \hbar B_{0} \\
& E_{ \pm \frac{1}{2}}=-\frac{e^{2} q Q}{4} \mp \frac{1}{2} \gamma \hbar B_{0},
\end{aligned}
$$

resulting in NQR transitions at frequencies of $\omega_{Q, \mp m}=\omega_{Q} \pm \omega_{0}$ where $\omega_{Q}=\frac{e^{2} q Q}{2 \hbar}$ and $\omega_{0}=\gamma B_{0}$. The energy levels are shown in Figure 2.1, and Figure 2.2 shows the energy levels for a spin $I=\frac{5}{2}$ nucleus. 


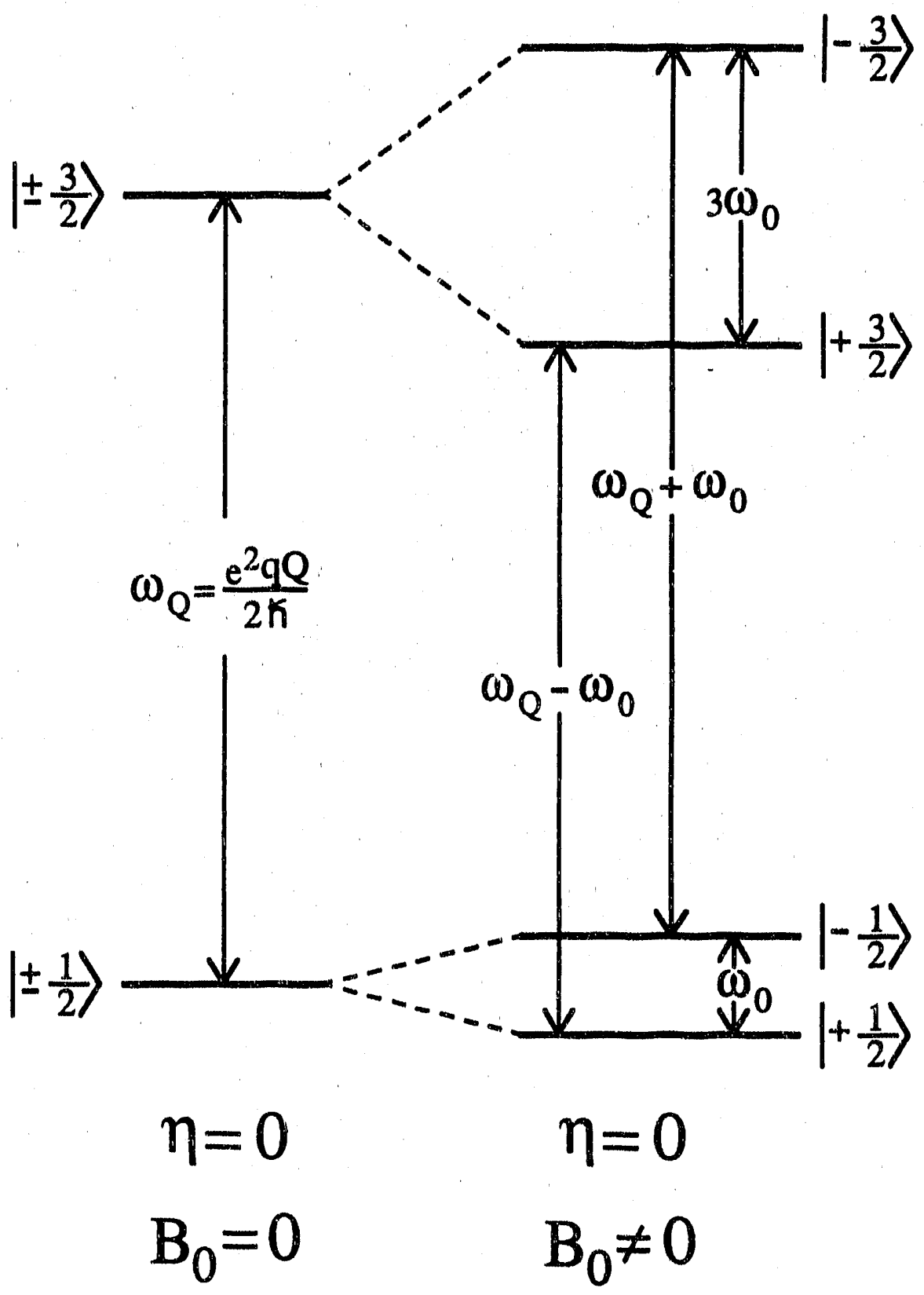

Figure 2.1: The energy levels of a spin $I=\frac{3}{2}$ nucleus in the absence of a magnetic field (left) and when the weak magnetic field is applied along the z-axis of the quadrupolar PAS (right). The asymmetry parameter $(\eta)$ is set to zero. Upon application of the magnetic field, the original pure NQR resonance at $\omega_{Q}$ is split symmetrically into two transitions at $\omega_{Q}-\omega_{0}$ and $\omega_{Q}+\omega_{0}$ where $\omega_{0}=\gamma B_{0}$. 


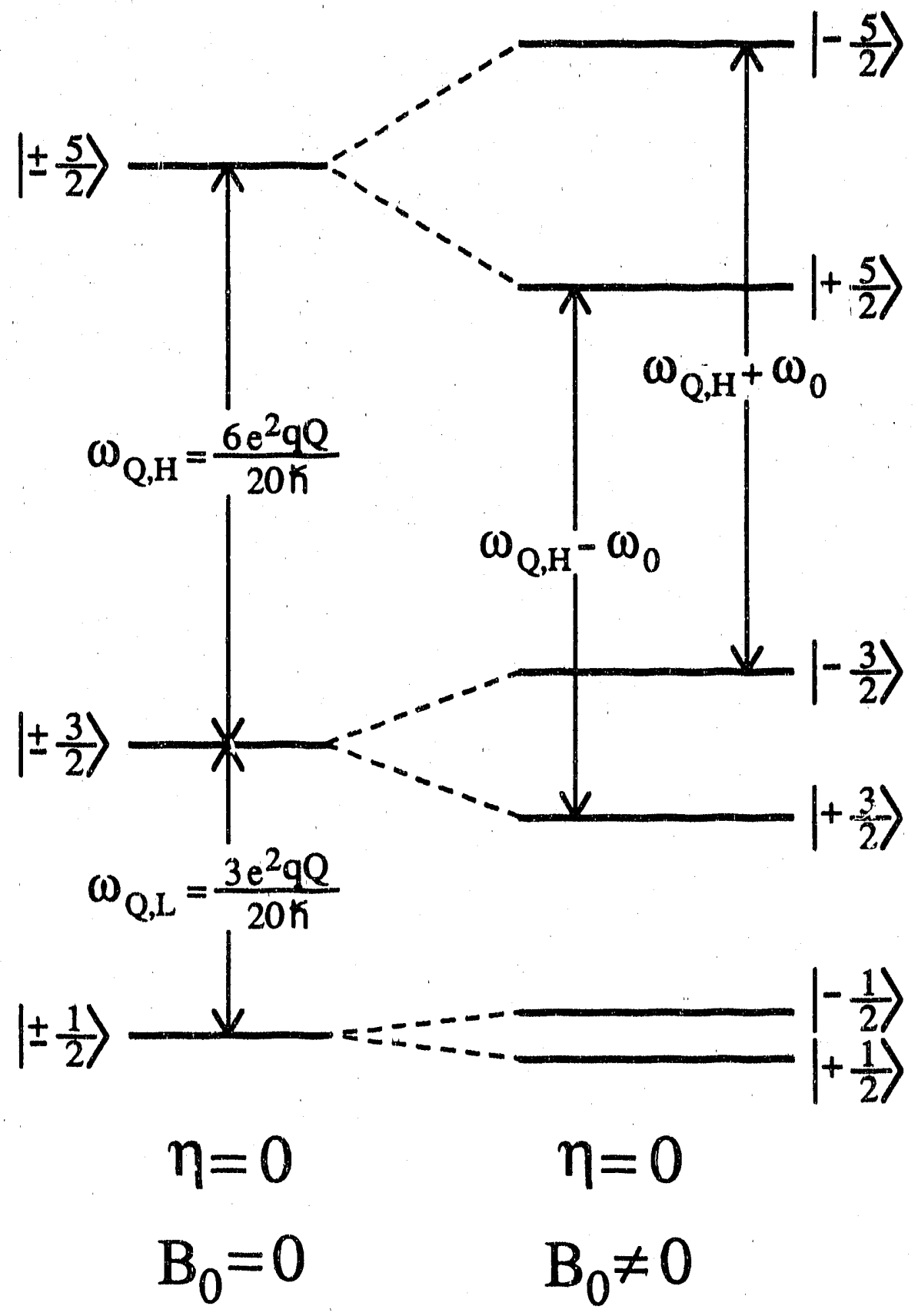

Figure 2.2: The energy levels of a spin $I=\frac{5}{2}$ nucleus in the absence of a magnetic field (left) and when the weak magnetic field is applied along the $z$-axis of the quadrupolar PAS (right). The asymmetry parameter $(\eta)$ is set to zero. The two original pure NQR resonances, $\omega_{Q, L}$ and $\omega_{Q, H}$, are each split into two transitions centered about the original resonances. 
The thermal equilibrium populations of the spin states, given by the hightemperature approximation [10], are

$$
\begin{aligned}
& P_{-\frac{3}{2}}=\frac{1}{4}\left(1-\frac{e^{2} q Q}{4 k T}-\frac{3 \gamma \hbar B_{0}}{2 k T}\right) \\
& P_{+\frac{3}{2}}=\frac{1}{4}\left(1-\frac{e^{2} q Q}{4 k T}+\frac{3 \gamma \hbar B_{0}}{2 k T}\right) \\
& P_{-\frac{1}{2}}=\frac{1}{4}\left(1+\frac{e^{2} q Q}{4 k T}-\frac{\gamma \hbar B_{0}}{2 k T}\right) \\
& P_{+\frac{1}{2}}=\frac{1}{4}\left(1+\frac{e^{2} q Q}{4 k T}+\frac{\gamma \hbar B_{0}}{2 k T}\right)
\end{aligned}
$$

The z-magnetization of the sample is calculated by summing the products of the populations of each level multiplied by its respective $I_{z}$ value,

$$
\begin{aligned}
M_{z} & =N \gamma \hbar \sum_{i}\left(P_{i} I_{z i}\right), \\
M_{z} & =N \gamma \hbar \sum_{i} P_{i} m_{i} .
\end{aligned}
$$

$N$ is the number density of spins, $m_{i}=\left\langle i\left|I_{z}\right| i\right\rangle$, and $i$ is the quantum number of the state. At thermal equilibrium, this gives a z-magnetization of

$$
M_{z}=\frac{N \gamma \hbar^{2}}{4 k T}\left(\frac{10}{2} \gamma B_{0}\right)
$$

Now we can look at the effects of an rf field as it is swept in frequency, from low to high frequency $[24,25]$. The $+\frac{1}{2} \leftrightarrow+\frac{3}{2}$ transition, at $\omega_{Q}-\omega_{0}$, occurs at a lower frequency than the $-\frac{1}{2} \leftrightarrow-\frac{3}{2}$ transition so is the first one to be irradiated. As the rf reaches the $+\frac{1}{2} \leftrightarrow+\frac{3}{2}$ transition it excites the spins and changes the populations of these two states. Assuming the rf field is strong enough to cause equalization of populations of the $+\frac{1}{2}$ and $+\frac{3}{2}$ states, we get the new populations

$$
P_{+\frac{3}{2}}^{\prime}=P_{+\frac{1}{2}}^{\prime}=\frac{1}{4}\left(1+\frac{\gamma \hbar B_{0}}{k T}\right)
$$


After saturating this transition $\left(+\frac{1}{2} \leftrightarrow+\frac{3}{2}\right)$, the induced z-magnetization of the sample is now

$$
M_{z}^{\prime}=\frac{N \gamma \hbar^{2}}{4 k T}\left(\frac{9}{2} \gamma B_{0}+\frac{1}{4} \frac{e^{2} q Q}{\hbar}\right) .
$$

Seeing that $\frac{e^{2} q Q}{\hbar}$ is always much larger than $\gamma B_{0}$, the sample magnetization changes dramatically when a NQR transition is excited.

As the sweep continues, the rf reaches the $-\frac{1}{2} \leftrightarrow-\frac{3}{2}$ transition and equalizes the populations of these two levels. Ignoring any decrease in sample magnetization from relaxation effects or other sources, this results in a final z-magnetization of

$$
M_{z}^{\prime \prime}=\frac{N \gamma \hbar^{2}}{4 k T}\left(\frac{8}{2} \gamma B_{0}\right)
$$

close to its original thermal equilibrium value. Thus, an idealized plot of the zmagnetization versus frequency of the rf yields a rectangular peak centered about $\omega_{Q}$, the pure NQR frequency, as shown in Figure 2.3.

Several factors, in practice, distort this rectangular peak. Firstly, the nuclei observed are usually dipolar coupled to neighboring spins and are usually subjected to some local variations in the electric field gradients. Relaxation effects, spin-spin and spin-lattice, also tend to alter the shape of the peak. Lastly, a major influence can be attributed to the use of powdered samples. This is discussed in a separate section.

Dipolar coupling of the nuclei of interest to other nuclei usually produces a manifold of energy levels about the original energy levels. Since the dipolar interaction has a $r^{-3}$ dependence, most of the energy levels remain fairly close to their original positions. This results in an approximately Gaussian absorption lineshape for each transition and smooths out the edges of the rectangular peak. The Gaussian absorption lineshapes have a characteristic width of approximately $\gamma B_{L}$, where $B_{L}$ is 


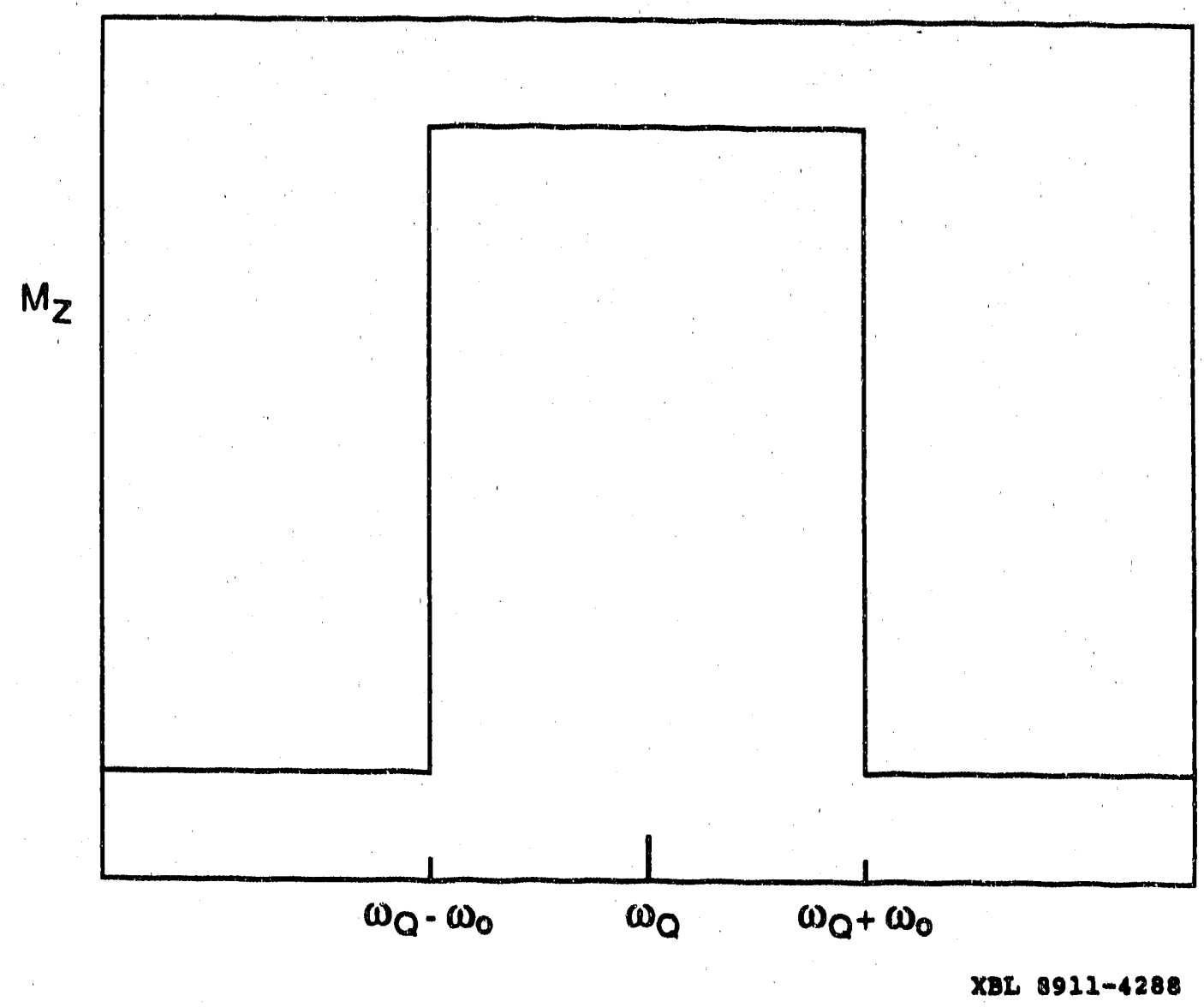

Figure 2.3: Theoretical z-magnetization signal, from a single crystal sample using z-axis cw NQR and ignoring all relaxation effects, expected from a rf frequency sweep through both transitions of a NQR resonance. The weak magnetic field is applied along the z-axis of the axially symmetric quadrupolar PAS. 
called the local field. Abragam and Kambe have calculated $B_{L}$ for a cubic lattice of $\operatorname{spin} I=\frac{3}{2}$ nuclei where the electric field gradient is along the cubic axis [26]. They found that

$$
B_{L} \doteq 60 \frac{\gamma^{2} \hbar^{2}}{d^{6}}
$$

where $d$ is the dimension of the unit cell. For most of the samples involved in this work, the local field is about a few gauss.

Small or large variations in the electric field gradients of the nuclear sites can alter the pure nuclear quadrupole resonance frequency. These electric field gradient variations usually arise from either site defects, small displacements, or noncrystalline structures such as glasses $[27,28]$. If the pure NQR frequency experiences a distribution of shifts, this will also broaden and smooth out the observed NQR resonance. Nuclei with large quadrupole moments $(e Q)$ experience a larger change in frequency than small quadrupole moment nuclei for the same amount of change in the electric field gradients, i.e. $\sigma\left(\nu_{Q}\right) \propto e Q$ and $\sigma\left(\nu_{Q}\right) \propto \sigma(e q)$.

Cross-relaxation between the $+m\left(+\frac{1}{2}\right.$ and $+\frac{3}{2}$ states $)$ and $-m\left(-\frac{1}{2}\right.$ and $-\frac{3}{2}$ states) manifolds, via spin-diffusion $[29,30]$, has the potential to drastically alter the shane and size of the signal; in the worst cases destroying the signal completely. After exciting the $+m$ manifold, and before reaching the $-m$ manifold, cross-relaxation and spin-lattice relaxation both tend to decrease the magnitude of the induced z-magnetization. This stems from the fact that cross-relaxation is relatively efficient when the two manifolds are close in energy. The cross-relaxation process makes it such that spins in the $+\frac{3}{2}$ state undergo stimulated emission to the $+\frac{1}{2}$ state while spins in the $-\frac{1}{2}$ go up to the $-\frac{3}{2}$ state. This process is a $\Delta m=-2$ transition for the spin system, which we will call a "flip-flip", and is mediated by 
the $F$ term of the dipolar Hamiltonian, Equation 2.13 [10]. Another process ("flopflop"), $\Delta m=+2$ transition, is also possible and this is mediated by the $E$ term of the dipolar Hamiltonian (Equation 2.12) to make a spin in the $-\frac{3}{2}$ state go to the $-\frac{1}{2}$ and a spin from the $+\frac{1}{2}$ state go to the $+\frac{3}{2}$ state. While each of these crossrelaxation processes, $\Delta m=+2$ or $\Delta m=-2$, has an equal absolute probability of occuring [31], the rates of these two processes are different in the middle of the sweep, Figure 2.4. This is because of the different populations of the states involved at that point. The rates of these processes can be written as

$$
\begin{aligned}
\frac{d M_{z}}{d t} & \propto-\left(+\frac{3}{2}\right) P_{+\frac{3}{2}}^{\prime} W+\left(+\frac{1}{2}\right) P_{+\frac{3}{2}}^{\prime} W-\left(-\frac{1}{2}\right) P_{-\frac{1}{2}} W+\left(-\frac{3}{2}\right) P_{-\frac{1}{2}} W \\
& \left.\propto-W\left(P_{+\frac{3}{2}}^{\prime}+P_{-\frac{1}{2}}\right), \text { (flip - flip process, decreases } M_{z}\right) \\
\frac{d M_{z}}{d t} & \propto+\left(+\frac{3}{2}\right) P_{+\frac{1}{2}}^{\prime} W-\left(+\frac{1}{2}\right) P_{+\frac{1}{2}}^{\prime} W+\left(-\frac{1}{2}\right) P_{-\frac{3}{2}} W-\left(-\frac{3}{2}\right) P_{-\frac{3}{2}} W \\
& \left.\propto+W\left(P_{+\frac{1}{2}}^{\prime}+P_{-\frac{3}{2}}\right), \text { (flop - flop process, increases } M_{z}\right)
\end{aligned}
$$

where $W$ is the absolute probability of a transition and $P_{i}$ is the population of the $i$ th state. Since, in between the two transitions, $P_{+\frac{3}{2}}^{\prime}=P_{+\frac{1}{2}}^{\prime}$ and $P_{-\frac{1}{2}}>P_{-\frac{3}{2}}$, the flip-flip term is larger and tends to decrease the induced overall magnetization. This just says that if there is a difference in the population differences between manifolds, cross-relaxation will equalize the population differences with some characteristic time $T_{C R}$. This cross-relaxation process is in many aspects similar to the techniques of double resonance $[18,32]$ and cross-polarization [33, 34].

The rate of the frequency sweep effectively determines the amount of time that cross-relaxation is allowed to occur. There is evidence in this work demonstrating that strong dipolar coupling tends to increase the cross-relaxation rate. It is possible to reduce the effects of cross-relaxation by either making the sweep rate faster or increasing the static magnetic field to the point where the cross-relaxation rate gets 


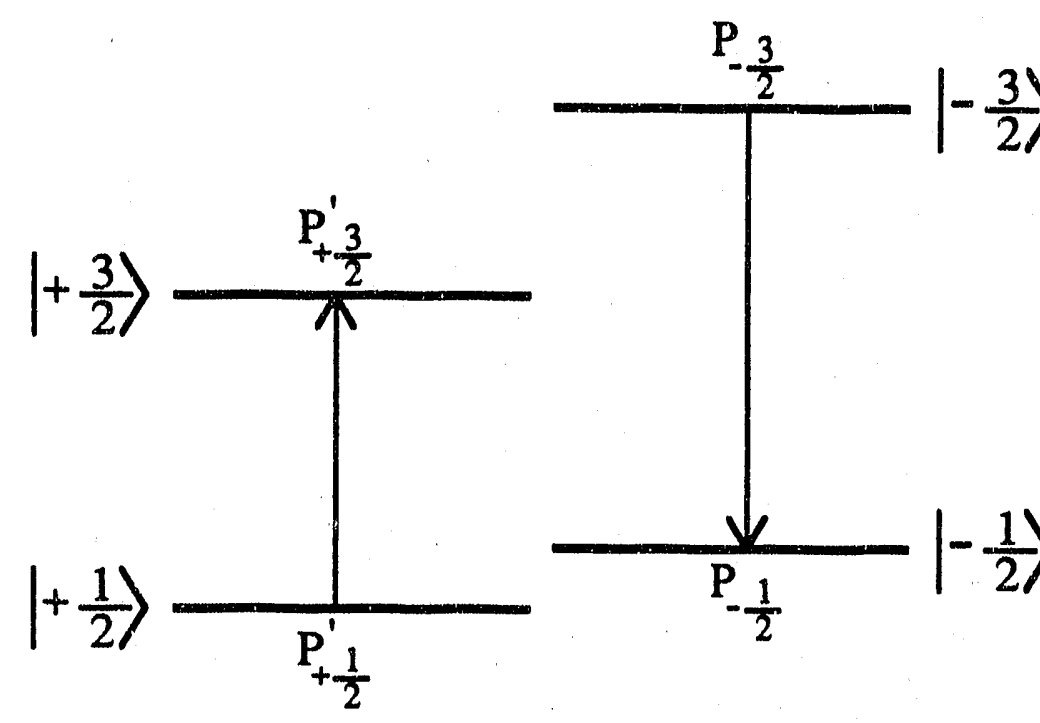

flop-flop, E term

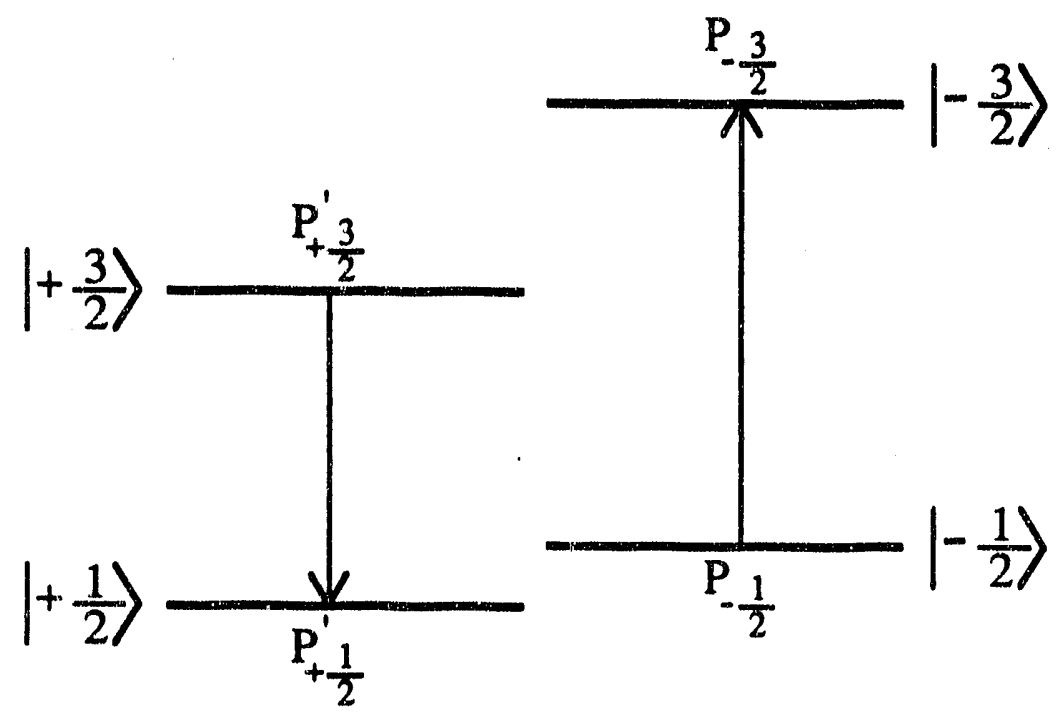

flip-flip, F term

Figure 2.4: A schematic illustration of the flop-flop and flip-flip processes, mediated by the $E$ and $F$ terms of the dipolar Hamiltonian, involved in the cross-relaxation of the $+m$ and $-m$ manifolds. The populations of the states are those after one transition $\left(+\frac{1}{2} \leftrightarrow+\frac{3}{2}\right)$ has been irradiated. These populations make the rate of the flip-flip process larger and thus tend to decrease the induced z-magnetization. 
small [25]. This point is usually where $B_{0} \approx 2 B_{L}$. However, one must take care because excessively large magnetic fields have their own disadvantages when applied to powder samples and one usually sets the magnetic field such that $B_{0} \approx B_{L}$.

Along with cross-relaxation effects, spin-lattice relaxation still exists and sometimes cannot be totally ignored. After passing through the low frequency transition and saturating it, spin-lattice relaxation tends to bring the populations of the $+\frac{3}{2}$ and $+\frac{1}{2}$ states back to their thermal equilibrium values. These relaxation processes cause the magnetization associated with these states to grow back in, possibly before the high frequency transition is reached. Some time before the rf saturates the high frequency transition, the sample magnetization could reach the initial baseline level. Then, as the rf saturates the high frequency transition, the z-magnetization would extend below the baseline before decaying back to its initial baseline value. The simulated lineshapes, of a single crystal sample, for various sweep rates are shown in Figure 2.5. The lineshapes were calulated by numerically approximating the integral

$$
S(\omega)=\int_{0}^{\omega}\left[g_{+m}\left(\omega^{\prime}\right)+g_{-m}\left(\omega^{\prime}\right)\right] e^{-\frac{\omega-\omega^{\prime}}{\kappa T^{\prime}}} d \omega^{\prime}
$$

$g_{ \pm m}\left(\omega^{\prime}\right)$ represent the Gaussian-like absorption lineshapes of each manifold, centered at $\omega_{Q} \pm \omega_{0}$, and have opposite signs corresponding to the different sign of $m$ in the two manifolds. Assuming the sample to be purely crystalline, the linewidth of the absorption lineshapes is determined by the strength of the dipolar coupling. The time constant $T^{\prime}$ accounts for both cross-relaxation and spin-laitice relaxation effects during the sweep, and $\kappa$ is the sweep rate. The simulated lineshapes are of the $\pm \frac{3}{2} \leftrightarrow \pm \frac{5}{2}$ transitions of ${ }^{27} \mathrm{Al}$ in $\alpha-\mathrm{Al}_{2} \mathrm{O}_{3}$. The experimen'al lineshapes are presented in Chapter 6. 


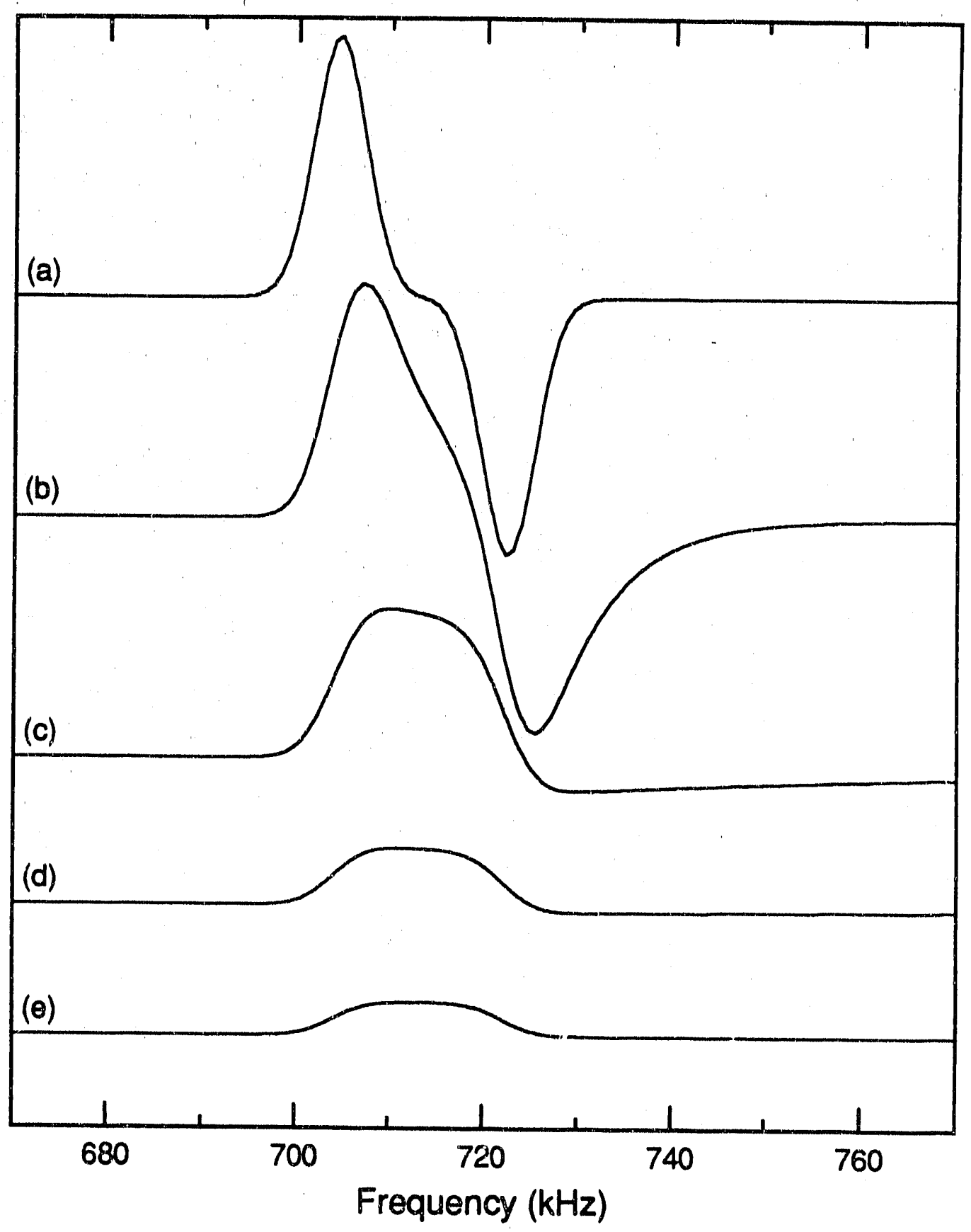

Figure 2.5: Simulated lineshapes of the $\pm \frac{3}{2} \leftrightarrow \pm \frac{5}{2}$ resonance of ${ }^{27} \mathrm{Al}$ in the $\alpha-\mathrm{Al}_{2} \mathrm{O}_{3}$ single crystal using forward sweeps of various $\mathrm{rf}$ sweep rates. The slowest sweeps are at the top and the fastest sweeps are at the bottom. The slowest sweep rate (a) shows a case where relaxation processes have returned the induced z-magnetization to its thermal equilibrium value, before the high frequency transition has been irradiated. 
The works of Dean, Hahn, and co-workers tell us that associated with each pair of energy levels, in each of the $+m$ or $-m$ manifolds, is a rotating magnetization [23, $35,36]$. These magnetizations precess about the z-axis of the PAS at the energy level splitting frequency. The sign of each magnetization and its direction of precession (left-hand or right-hand) depend on the sign of the manifold. They show that it is possible to selectively irradiate one transition of a NQR resonance using circularly polarized rf. An example of this is shown using the $+\frac{3}{2} \leftrightarrow+\frac{5}{2}$ and $-\frac{3}{2} \leftrightarrow-\frac{5}{2}$ transitions of ${ }^{27} \mathrm{Al}$ in a single crystal of $\alpha-\mathrm{Al}_{2} \mathrm{O}_{3}$ in Chapter 6.

So far we have considered only the case of a saturating rf field. Under optimum circumstances, the experimentalist may be able to specify the sweep parameters such that the sweep satisfies the conditions for adiabatic rapid passage. Adiabatic rapid passage requires that one sweep through the resonance in less than $T^{\prime}$ and that the magnetization be aligned along the effective magnetic field during the sweep, the effective field being the sum of the rf and static magnetic fields in the rotating frame. These conditions can be expressed as

$$
\left(\gamma B_{1}\right)^{2} \gg \frac{d \nu}{d t} \gg \frac{\Delta \nu}{T^{\prime}}
$$

where $B_{1}$ is the strength of the rf field, $\frac{d \nu}{d t}$ is the sweep rate, and $\Delta \nu$ is the resonance linewidth. If the sweep parameters satisfy the adiabatic rapid passage conditions, then the populations of the $+\frac{1}{2}$ and $+\frac{3}{2}$ states will be inverted. This corresponds to an inversion of the magnetization associated with these two states, resulting in an induced z-magnetization approximately twice as large as that in the case of saturation. Equation 2.30 tells us that strong rf fields may be necessary to satisfy the adiabatic rapid passage conditions. It is usually the rf field coupling to the SQUID which determines the maximum strength of $\mathrm{rf}$ which can be applied to 
the sample. Since the SQUID could only tolerate of of at most $200 \mathrm{mG}$ in these experiments, none of the spectra were acquired using adiabatic rapid passage.

\subsubsection{Adaptations for Powder Samples}

The last, and most difficult to quantify, factor to affect the lineshape of a resonance is the use of a powder sample. The change in the lineshape is highly dependent on the sample parameters as well as the experimental conditions under which the spectrum was obtained. This section qualitatively covers z-axis NQR of powder samples. One of the factors which ultimately determines the practicality of a magnetic resonance technique is its amenability to study powder samples, since single crystal samples are quite suitable to high-field NMR techniques [37, 38].

In powder and amorphous samples, the z-axes of the quadrupolar principal axis systems are randomly oriented in direction and most of them do not align along the direction of the applied static magnetic field. Assuming the crystallites to be purely crystalline, all the nuclei of interest still have the same pure NQR frequency with a linewidth determined by the strength of the dipolar coupling. Application of a small static magnetic field will broaden these resonances as described below.

Using Equation 2.4 and degenerate perturbation theory, one calculates that the energy levels for the $\pm \frac{3}{2}$ states are shifted by

$$
\Delta E\left( \pm \frac{3}{2}\right)=\mp \gamma \hbar B_{0} m \cos \theta
$$

Equation 2.4 has been truncated to $H_{M}=-\gamma \hbar B_{0} I_{z} \cos \theta$ by the quadrupolar Hamiltonian to arrive at this result and the quantum numbers $(m)$ for these two states are still good for an axially symmetric electric field gradient. This is not true however for the $\pm \frac{1}{2}$ states which are mixed by the $I_{x}$ and $I_{y}$ terms of Equation 2.4 [5]. This 
mixing brings about two new states given by

$$
\begin{aligned}
& |+\rangle=+\sin \alpha\left|+\frac{1}{2}\right\rangle+\cos \alpha\left|-\frac{1}{2}\right\rangle, \\
& |-\rangle=-\cos \alpha\left|+\frac{1}{2}\right\rangle+\sin \alpha\left|-\frac{1}{2}\right\rangle,
\end{aligned}
$$

where $\tan \alpha=\left[\frac{f+1}{f-1}\right]^{\frac{1}{2}}$ and $f=\left[1+\left(I+\frac{1}{2}\right)^{2} \tan ^{2} \theta\right]^{\frac{1}{2}}$. The original quantum numbers of these states are in general no longer valid and this allows for transitions between either of these two states and either the $\pm \frac{3}{2}$ states. The resulting energy level shifts of the $| \pm\rangle$ states are given by $\Delta E( \pm)=\mp\left(\frac{f}{2}\right) \gamma \hbar B_{0} \cos \theta$.

To simulate lineshapes of powder samples, the absorption lineshapes of each manifold in Equation 2.29 must be modified to represent that of a powder sample. These absorption lineshapes depend not only on the angle between the z-axis of the crystallites with respect to the static magnetic field, but also on the strength of both the static and rf magnetic fields. This dependence can be expressed as

$$
g_{ \pm m}\left(\omega^{\prime}, B_{0}, B_{1}\right)=\frac{1}{4 \pi} \int_{0}^{2 \pi} d \phi \int_{0}^{\pi} g_{ \pm m}\left(\omega^{\prime}, \theta\right) \Gamma(\theta) \Psi\left(\theta, B_{1}\right) \Theta\left(\theta, B_{0}\right) \rho \sin \theta d \theta
$$

$g_{\text {土m }}\left(\omega^{\prime}, \theta\right)$ are Gaussians centered at $\omega_{Q} \pm \omega_{0} f \cos \theta$ representing the absorption lineshapes of each crystallite. The linewidths of the $g_{ \pm m}\left(\omega^{\prime}, B_{0}, B_{1}\right)$ for glassy and amorphous samples can be very large and possibly make the contribution from dipolar coupling negligible.

Since the induced magnetization appears along the z-axis of the quadrupolar principal axis system for each crystallite and we only detect magnetization along the z-axis of the lab frame, the signal from a crystallite with a polar angle $\theta$ with respect to the lab frame, is reduced by the factor $\Gamma(\theta) \propto \cos \theta$. The rf radiation is most effective in inducing transitions when it is applied orthogonally to the z-axis 
of the quadrupole principal axis system $[35,36]$. This will not be the case for many of the crystallites in a powder sample. So the $\Psi\left(\theta, B_{1}\right)$ term takes into account that only the projection of the rf perpendicular to the quadruplar z-axis is effective in inducing transitions. From our studies of a single crystal of $\alpha-\mathrm{Al}_{2} \mathrm{O}_{3}$, we find that the $\Psi\left(\theta, B_{1}\right)$ term, which describes the signal height as a function of $\mathrm{rf}$ strength, has the approximate form

$$
\Psi\left(\theta, B_{1}\right) \sim 1-e^{-\frac{B_{1, \rho f f}}{B_{1,0}}}
$$

The effective rf field is $B_{1, e f f}=B_{1} \cos \theta$, and $B_{1,0}$ is some characteristic rf strength which depends on the sample parameters and the sweep rate.

The small static magnetic field used to split the degeneracy of the $\omega_{Q,+m}$ and $\omega_{Q,-m}$ components, reduces the cross-relaxation rate between the $+m$ and $-m$ manifolds. The effective field, $B_{0, e f f}=B_{0} \cos \theta$, decreases in crystallites for which the z-axis is tilted away from the magnetic field axis. The $\Theta\left(\theta, B_{0}\right)$ term accounts for the decreased effectiveness of the dc field in reducing the cross-relaxation rate when the quadrupolar z-axis is not aligned along the magnetic field. The result is a reduction in signal intensity and a distortion of the lineshape, when the effective field approaches the local field $B_{L}$ (due to dipolar coupling) of the sample. We found that, for a single crystal of $\alpha-\mathrm{Al}_{2} \mathrm{O}_{3}, \Theta\left(\theta, B_{0}\right)$ can be roughly represented [39] by

$$
\Theta\left(\theta, B_{0}\right) \sim 1-e^{-\frac{B_{0, e f}}{B_{L}}} .
$$

The overall magnitude of $\Theta\left(\theta, B_{0}\right)$ is determined by the sweep rate and zero field cross-relaxation rate.

It is possible for the magnetic field to broaden the resonance since the splitting due to this field depends on the orientation of the crystallite. Assuming a purely 
crystalline powder sample and $B_{0}>B_{L}$, the linewidth will be around $2 \gamma B_{0}$. In most cases, one is able to set $B_{0} \approx B_{L}$ to minimize the linewidth and still maintain reasonable signal intensity. When studying amorphous samples, the distribution in sites usually dominates the linewidth and so the value of $B_{0}$ is not critical. If one were interested in the natural (zero-field) linewidth of the resonance, a plot of resonance linewidth versus applied magnetic field could be extrapolated to zero applied field.

The factor $\rho$ accounts for the filling factor of the sample which is less for a powder sample compared to a single crystal sample occupying the same volume. An indepth study was not performed on this matter, but estimates that $\rho \approx \frac{1}{2}$ appear reasonable when the number of particles is large and of an approximately spherical shape. The simulated lineshapes, Figure 2.6, are of the $\pm \frac{3}{2} \leftrightarrow \pm \frac{5}{2}$ transitions of ${ }^{27} \mathrm{Al}$ in $\alpha-\mathrm{Al}_{2} \mathrm{O}_{3}$ powder. The experimental lineshapes are presented in Chapter 6.

The extension of this experimental technique to nuclei with higher half-oddinteger spin $\left(I=m+\frac{1}{2}, m=2,3, \ldots\right)$ is relatively straightforward. In all these cases, the $\pm \frac{1}{2} \leftrightarrow \pm \frac{3}{2}$ transitions behaves just as described above, along with the fact that there are other energy levels above the $\pm \frac{3}{2}$ levels. Thus, in a spin $I=\frac{5}{2}$ nucleus, there are two pure NQR resonances, the $\pm \frac{1}{2} \leftrightarrow \pm \frac{3}{2}$ resonance and the $\pm \frac{3}{2} \leftrightarrow \pm \frac{5}{2}$ resonance. It is easier to deal with the latter transitions since there is no mixing of states by the magnetic field and the quantum numbers are all still good in an axially symmetric electric field gradient. The z-magnetizations that can theoretically be induced for spin $I=\frac{3}{2}$ and $I=\frac{5}{2}$ nuclei are presented below, Table 2.1, assuming a saturating rf sweep from low to high frequency. The final z-magnetization, when exciting either the $\pm \frac{1}{2} \leftrightarrow \pm \frac{3}{2}$ or $\pm \frac{3}{2} \leftrightarrow \pm \frac{5}{2}$ resonance of 


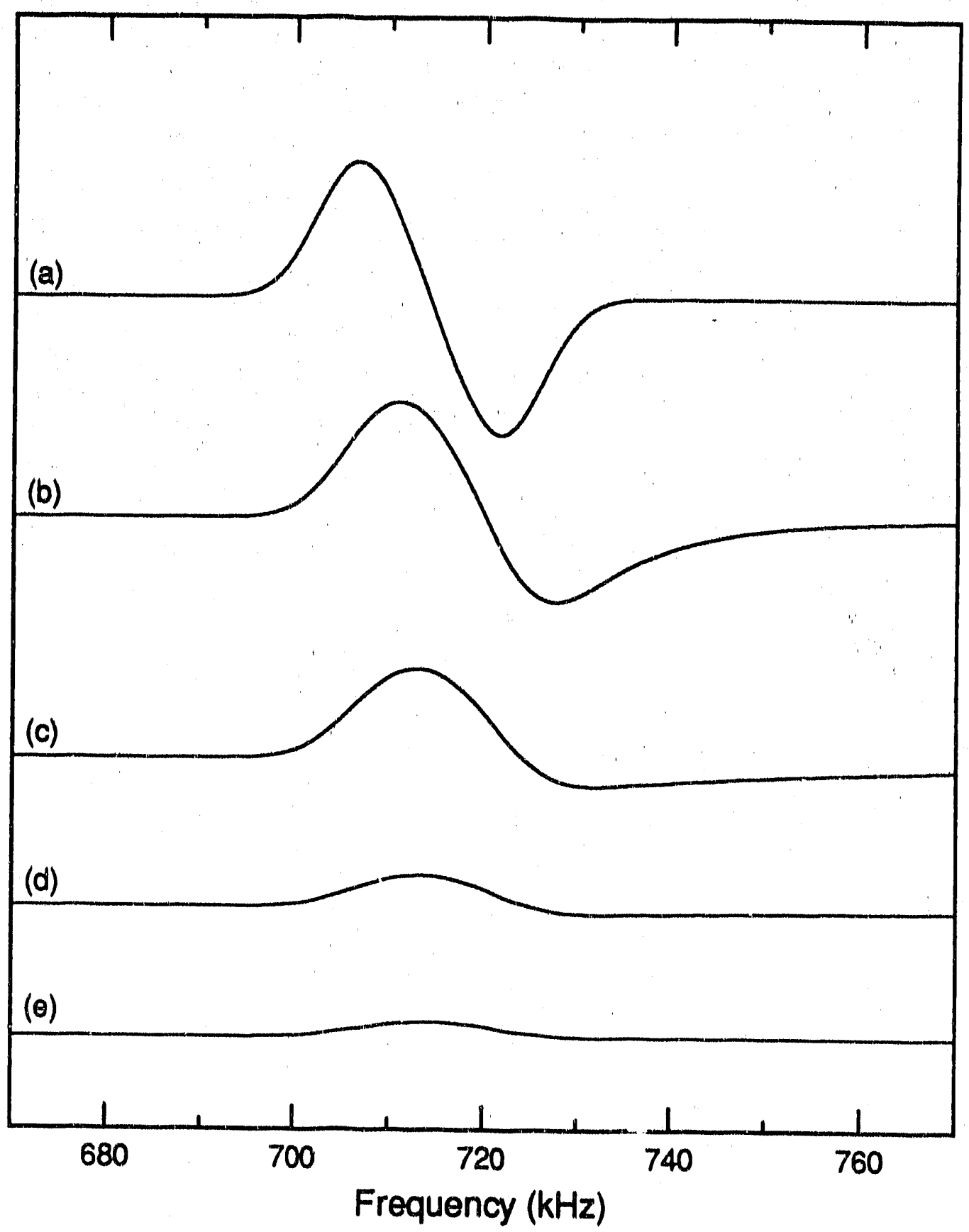

Figure 2.6: Simulated lineshapes of the $\pm \frac{3}{2} \leftrightarrow \pm \frac{5}{2}$ resonance of ${ }^{27} \mathrm{Al}$ in the $\alpha-\mathrm{Al}_{2} \mathrm{O}_{3}$ powder using forward sweeps of various if sweep rates. The slowest sweeps are at the top and the fastest sweeps are at the bottom. 


\begin{tabular}{|c|c|c|}
\hline \multirow[b]{2}{*}{ Z-Magnetization } & \multicolumn{2}{|c|}{ Spin of Nucleus } \\
\hline & $I=\frac{3}{a}$ & $I=\frac{5}{2}$ \\
\hline Thermal Equilibrium & $\frac{N \gamma h^{2}}{4 k T}\left[\frac{10}{2} \gamma B_{0}\right]$ & $\frac{N \gamma \AA^{2}}{6 t}\left[\frac{35}{2} \gamma B_{0}\right]$ \\
\hline $\begin{array}{c}\text { After Saturating } \\
+\frac{1}{2} \leftarrow+\frac{3}{2} \text { Transition } \\
\end{array}$ & $\frac{N \gamma h^{2}}{d k T}\left[\frac{9}{2} \gamma B_{0}+\frac{1}{4} \frac{a^{2} q Q}{h}\right]$ & $\frac{N \gamma h^{2}}{d x}\left[17 \gamma B_{0}+\frac{3}{d \delta} \frac{a^{2} g Q}{h}\right]$ \\
\hline $\begin{array}{c}\text { After Saturating } \\
+\frac{3}{2} \leftrightarrow+\frac{5}{2} \text { Transition } \\
\end{array}$ & & $\frac{N \gamma A^{2}}{6 k T}\left[17 \gamma B_{0}+\frac{6}{40} \frac{a^{2} g Q}{\hbar}\right]$ \\
\hline $\begin{array}{l}\text { After Saturating Both } \\
\text { Transitions of Resonance }\end{array}$ & $\frac{N \gamma h^{2}}{4}\left[\frac{8}{2} \gamma B_{0}\right]$ & $\frac{N \gamma n^{2}}{6 x T}\left[\frac{33}{2} \gamma B_{0}\right]$ \\
\hline
\end{tabular}

Table 2.1: Calculated z-magnetizations for spin $I=\frac{3}{2}$ and $I=\frac{5}{2}$ nuclei when saturating various transitions of a NQR resonance. The final z-magnetization when irradiating either the $\pm \frac{1}{2} \leftrightarrow \pm \frac{3}{2}$ resonance or the $\pm \frac{3}{2} \leftrightarrow \pm \frac{5}{2}$ resonance of a $\operatorname{spin} I=\frac{5}{2}$ nuclei is the same. 
a spin $I=\frac{5}{2}$ nucleus, is the same. When considering a reverse sweep (high to low frequency), a few modifications to the above equations need to be made. In a reverse sweep, the order in which the two components of the resonance are saturated is reversed, so the sign of the quadrupolar term in Equation 2.24 must be reversed. The sign of the Zeeman term remains unchanged. Since the magnitude of the quadrupolar term is normally much larger than the Zeeman term, the sign of the resonance peak is reversed on a reverse sweep. This property provides a convenient way to distinguish real signal from instrumental artifacts which generally do not change sign when the sweep direction is switched.

\subsubsection{Miscellaneous Considerations}

Several points regarding the comparison of single crystal and powder samples should be made clear in addition to the above discussion. Assume that the single crystal and the crystallites of the powder are purely crystulline, so that the only natural source of line-broadening is dipolar coupling. For a given static magnetic field such that $B_{0} \approx B_{L}$ and a fast sweep, the $\pm \frac{3}{2} \leftrightarrow \pm \frac{5}{2}$ resonance is about one and a half times wider than the natural linewidth in a single crystal. The $\pm \frac{3}{2} \leftrightarrow \pm \frac{5}{2}$ resonance of the powder sample is slightly narrower than the same resonance for the single crystal, a rather unique situation in magnetic resonance. However, the $\pm \frac{1}{2} \leftrightarrow \pm \frac{3}{2}$ resonance of the powder sample is about 3 times wider than the $\pm \frac{3}{2} \leftrightarrow \pm \frac{5}{2}$ resonance of the sarne powder sample. Examples of these are shown using $\alpha-\mathrm{Al}_{2} \mathrm{O}_{3}$ in Chapter 6.

When considering amorphous or glassy samples, variations in the electric field gradients cause the pure NQR resonance to broaden, even to the extent that these 
variations, and not the static magnetic fleld, determine the linewidth of the resonance. In this case, the lineshape and linewidth of the resonance observed by this SQUID technique will very closely approximate that determined by conventional pure NQR. An example of this is shown using $\mathrm{B}_{2} \mathrm{O}_{3}$ glass in Chapter 5 .

Using this z-axis NQR technique, relaxation processes during the sweep tend to destroy the symmetry of the lineshape for a single sweep direction. Since the peak in a reverse sweep has the opposite sign of the peak in a forward sweep, we subtract the reverse sweep from the forward sweep to get the final spectrum. Doing so has a 3-fold benefit. The subtraction of sweeps results in a lineshape that is symmetric with respect to $\omega_{Q}$ and eliminates the need to consider relaxation effecto when determining the value of ' $\omega_{Q}$. Secondly, as in any continuous wave (CW) method, the system (sample and instrument) has a response time to excitation and changes. By taking a difference spectrum, the experimontalist does not bias any particular sweep direction, both of which are equally accurate. The third advantage is that since instrumental artifacts in this technique do not change sign when the sweep direction is altered, it provides for an easy method in eliminating these artifacts. All the spectra shown in this work are reverse sweeps subtracted from forward sweeps, unless otherwise noted. Also, all the spectra shown were acquired using linearly polarized $\mathrm{rf}$, unless otherwise noted.

All of the preceeding discussion utilized an axially symmetric electric field gradient to simplify the discussion. In this section, I try to describe what happens in a non-axially symmetric electric field gradient. When it is a half-odd-integer spin quadrupolar nucleus in zero magnetic field, the energy levels of the $\pm m$ states are always degenerate, regardless of whether the electric field gradient is axially 
symmetric or non-axially symmetric. This feature is $d x$ : to the fact that fermions (spin $I=m+\frac{1}{2}, m=0,1,2, \ldots$ ) involved in a quadrupolar interaction exhibit timereversal symmetry; that is, reversing all velocities and angular momenta leaves the system with the same equations of motion $[40,41,42,43]$. A consequence of this time-reversal symmetry is Kramers degeneracy which tells us that each energy level of the system must be at least two-fold degenerate no matter huw complicated the electric fields maay be. Bosons ( $\operatorname{spin} I=m, m=0,1,2, \ldots$ ) in a quadrupolar interaction, however, do not exhibit time-reversal symmetry and so no such degeneracy is required when the electric field gradient is asymmetrical. The application of a small magnetic field breaks Kramers degeneracy for half-odd-integer spin nuclei. This splits the $\pm m$ states symmetrically about their original levels. This allows for the easy determination of pure NQR resonance frequencies, even for non-axially symmetric electric field gradients. However, a non-axially symmetric electric field gradient will cause state mixing even in zero magnetic field [5]. Standard perturbation theory shows that the new states $\left|m^{\prime}\right\rangle$ will retain character of its $|m\rangle$ state and have some admixtures of the $|m-2\rangle$ and $|m+2\rangle$ states, for example

$$
\left|+\frac{1^{\prime}}{2}\right\rangle=a\left|+\frac{1}{2}\right\rangle+b\left[\left|-\frac{3}{2}\right\rangle,\left|+\frac{5}{2}\right\rangle\right] \text {. }
$$

The effect of this is to alter the eigenvalues of the states $\left|m^{\prime}\right\rangle$ with respect to the operator $I_{z}$ and change the signal intensity. Das and Hahn have also calculated the transition probabilities under this state-mixing. The overall effect is the reduction of signal intensity as the electric field gradient becomes more non-axially symmetric. No attempt was made to explicitly calculate this reduction in signal intensity. 


\subsection{NQR Resonance Frequencies}

In general, one cannot determine $\frac{e^{2} q Q}{\hbar}$ and $\eta$ separately for spin $I=\frac{3}{2}$ nuclei from pure NQR. For spin $I=\frac{5}{2}$ nuclel, $\frac{e^{2} q Q}{\hbar}$ and $\eta$ can be evaluated separately since there are two pure quadrupolar resonances. For both the single crystal and powder samples, the transitions in a small magnetic field are centered about the pure quadrupolar resonance frequencies. For spin $I=\frac{3}{2}$, the pure NQR frequency

$$
\omega_{Q}=\frac{e^{2} q Q}{2 \hbar}\left(1+\frac{\eta^{2}}{3}\right)^{\frac{1}{2}}
$$

is well known. For spin $I=\frac{5}{2}$ nuclei, there are two pure NQR resonances, $\pm \frac{1}{2} \leftrightarrow$ $\pm \frac{3}{2}$ and $\pm \frac{3}{2} \leftrightarrow \pm \frac{5}{2}$. The usual approach to solving for the energy levels, and thus resonance frequencies, has been a purely numerical solution. An analytical solution is presented here and its derivation is shown in Appendix A. The $\pm \frac{1}{2} \leftrightarrow \pm \frac{3}{2}$ resonance occurs at

$$
\omega_{Q, L}=\frac{e^{2} q Q}{20 \hbar} r^{\frac{1}{3}}(2 \sqrt{3}) \sin \left(\frac{\theta}{3}\right)
$$

while the $\pm \frac{3}{2} \leftrightarrow \pm \frac{5}{2}$ resonance occurs at

$$
\omega_{Q, H}=\frac{e^{2} q Q}{20 \hbar} r^{\frac{1}{3}}\left[3 \cos \left(\frac{\theta}{3}\right)-\sqrt{3} \sin \left(\frac{\theta}{3}\right)\right]
$$

where

$$
\begin{aligned}
r^{\frac{1}{3}} & =\left(\frac{7}{3} \eta^{2}+7\right)^{\frac{1}{2}} \\
\theta & =\arctan \left[\frac{-P}{10\left(\eta^{2}-1\right)}\right]
\end{aligned}
$$

and

$$
P=\left[\frac{343}{27} \eta^{6}+\frac{43}{3} \eta^{4}+543 \eta^{2}+243\right]^{\frac{1}{2}} .
$$

The expression for the ratio $\left(\frac{\omega_{Q, H}}{w_{Q, L}}\right)$ simplifies to an equation as a function of $\eta$ only. After the proper experimental determination of $\omega_{Q, L}$ and $\omega_{Q, H}$, they can be used to 
search for the value of $\eta$ which satisfies this function of $\eta$ only. The $\frac{e^{2} q Q}{\hbar}$ can then be determined using either the equation for $\omega_{Q, L}$ or $\omega_{Q, H}$. It appears to the author that these analytical expressions for the resonance frequencies, derived by solving for the roots of the cubic secular equation, have not been previously published.

There are various methods of determining the pure NQR resonance frequency from the forward minus reverse (forward-reverse) spectrum. One may choose the peak maximum as the resonance frequency or use the center of mass of the resonance. An alternative is to use the average of the edges of 8 , resunance. 


\section{Chapter 3}

\section{General Principles of the dc SQUID System}

\subsection{Introduction}

This section gives a qualitative description of how the dc SQUID measures magnetic flux and how the flux through it affects the voltage across the SQUID. Figure 3.1 is a schematic of a dc SQUID (Superconducting QUantum Interference Device), which consists of a pair of Josephson junctions connected in parallel. These Josephson junctions are usually a thin layer of insulating material separating two superconductors $[44,45]$. SQUIDs usually have dimensions on the order of millimeters $[46,47]$. The SQUID, along with its lock-in detection, feedback electronics, filters, and preamplifiers will be referred to as the SQUID system. The output voltage of the SQUID system should not be confused with the voltage across the SQUID, taken between points A and B in Figure 3.1.

A fixed bias current $\left(j_{B}\right)$ is forced through the loop formed by the pair of Joseph- 

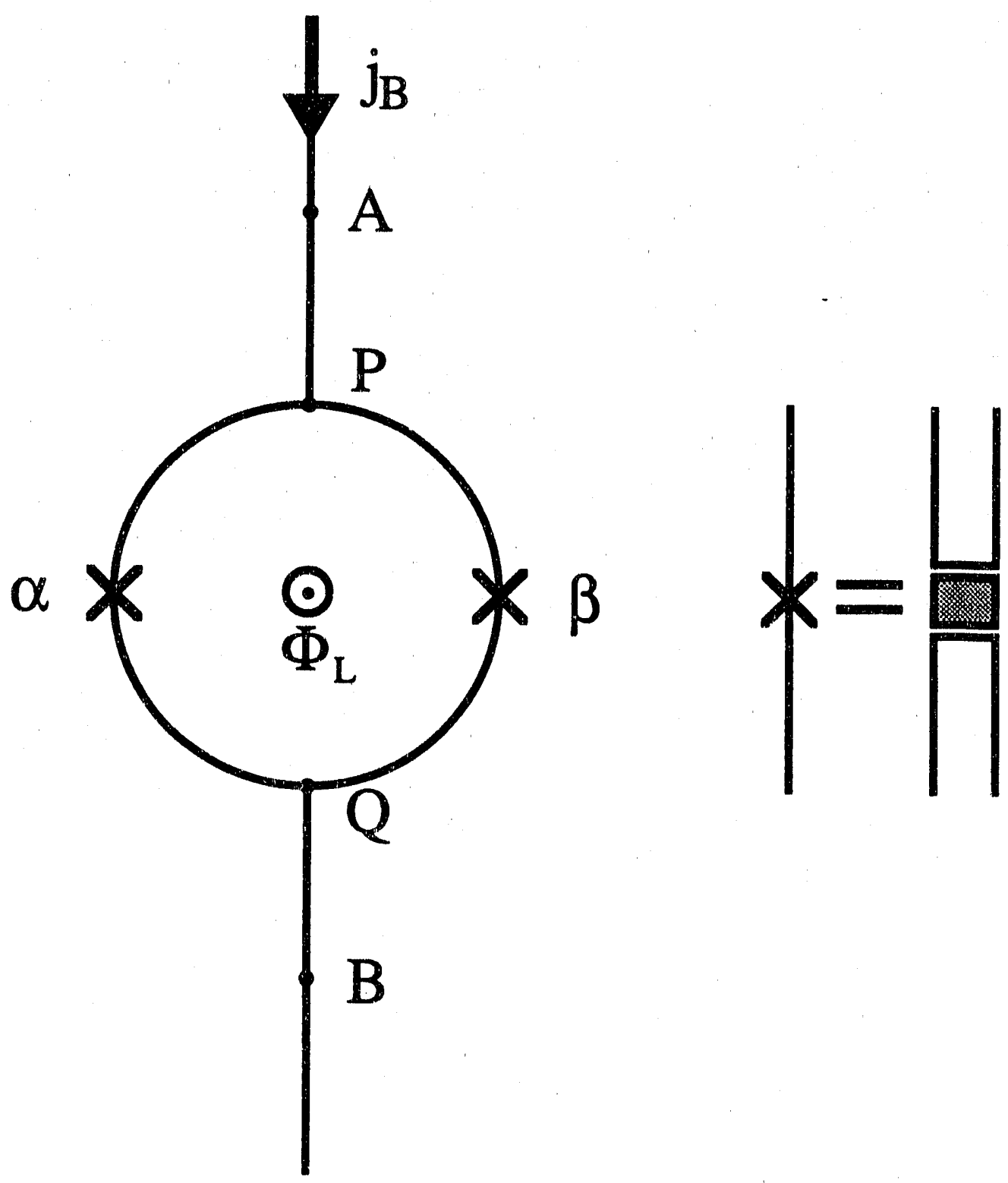

Figure 3.1: Schematic of a dc SQUID (left) and a Josephson junction (right). The dc SQUID is made by connecting two Josephson junctions in parallel. As superconducting electron pairs travel through either path $(\mathrm{P} \alpha \mathrm{Q}$ or $\mathrm{P} \beta \mathrm{Q})$ and tunnel through the junctions, they acquire a phase which depends on the magnetic flux threading through the loop $\left(\Phi_{L}\right)$. Quantum interference, between electron pairs traversing junctions $\alpha$ and $\beta$, produces a periodic voltage across the dc SQUID as a function of $\Phi_{L}$. 
son junctions. Some of this bias current consists of supercurrent and the remainder flows as normal current. The conventional picture of superconductivity has the supercurrent made up of bound pairs of electrons and no voltage is developed by this flow of superconducting electron pairs $[48,49,50]$. The normal current consists of the flow of individual electrons and, as usual, causes a voltage across the SQUID. This voltage is, thus, proportional to the fraction of the bias current that flows as normal current.

The voltage across the SQUID, at a fixed bias current, depends on the amount of magnetic flux threading through the loop formed by the SQUID. This is because the magnetic flux determines the amount of supercurrent, and thus the amount of normal current, and consequently the voltage across the SQUID. The mechanism by which the magnetic flux affects the amount of supercurrent is as follows. These superconducting electron pairs accumulate a phase shift, when passing through a junction, proportional to the flux thror:gh the SQUID. Assuming that the two halves of the SQUID are identical, the phase shift of the electron pairs passing through the junction $\alpha$ are of equal magnitude, but of opposite sign, as the phase shift for the electron pairs passing through junction $\beta$. Should the two supercurrents arrive at point $Q$ with a phase difference of $(2 n+1) \pi$ radians, they will interfere destructively and the total supercurrent through the SQUID will be zero. All of the bias current, in this case, will consist of normal current and the voltage across the SQUID will be maximized. On the other hand, the magnitude of the magnetic flux through the SQUID may be such that the phase difference between the two supercurrents at point $\mathrm{Q}$ is $(2 n) \pi$ radians. In this case, there will be constructive interference, the total supercurrent will be maximized, and the voltage across the SQUID will 
be minimized. The bias current through the SQUID is usually set greater than the maximum supercurrent possible through the two junctions in parallel, such that the voltage across the SQUID is never zero and some current always flows as normal current.

The result of this is that the voltage across the SQUID is a periodic function of the amount of magnetic flux threading through the loop. Most SQUID systems are made to operate in the flux-locked mode, making the SQUID itself a null detector [20]. In this operating mode, the output voltage of the SQUID system is proportional to the amount of current required to keep the total flux through the SQUYD loop fixed using a modulation/feedback coil. This has the advantage of a linear system response to the input and makes it unnecessary to keep track of exactly where one is on the voltage $(V)$ versus flux $(\phi)$ curve. In the next section, the equations describing the inteference effect of the SQUID are presented. This is followed by discussions on the transfer of magnetic flux from the sample to the SQUID, and on the flux-locked mode operation.

\subsection{Equations Describing the dc SQUID}

First, we will take a look at the behavior of a single Josephson junction. The Bardeen-Cooper-Schrieffer (BCS) theory of superconductivity states that all the bound electron pairs in a superconductor are phase coherent, i.e. their wavefunctions have the same phase $[48,49]$. This allows the wavefunctions of all the electron pairs to be described by a single wave function for the entire superconductor. When two pieces of superconducting material are brought close to each other, some overlap of the two superconductor wavefunctions occurs [51]. This results in the tunneling 
of electron pairs from one superconductor to the other, and makes the phases of the two superconductor wavefunctions related as shown in Figure 3.2. The tunneling allows a supercurrent to flow and its amplitude depends sinusoidally on the phase difference across the junction according to

$$
j_{s}=j_{1} \sin [\delta(t)]
$$

where

$$
\delta(t)=\delta(0)+\frac{2 e}{\hbar} \int_{0}^{t} V\left(t^{\prime}\right) d t^{\prime}
$$

The $\delta(0)$ term is a constant phase difference and is of no particular interest. The second term makes the total phase time dependent, where $V(t)$ is the voltage across the junction and $e$ is the electronic charge of a single electron.

The dc SQUID is usually biased with a current a few microamps greater than the maximum supercurrent possible. With the effective resistance of a SQUID being on the order of ohms, this leads to a voltage of many microvolts across the SQUID. This tells us that under normal circumstances, the supercurrent oscillates at gigahertz frequencies. For example, at a fixed voltage of one microvolt across the SQUID, the supercurrent oscillates at the Josephson frequency,

$$
\nu=\frac{2 e V}{h}
$$

of around $486 \mathrm{MHz}$. This can be used to obtain very accurate values for the ratio $\frac{e}{\hbar}[44,52]$. However, since the SQUID is usually current biased, the voltage across the SQUID will depend on the supercurrent. This makes the expression for the oscillating supercurrent more complicated. We do not need to be concerned with this though, since only the time-average value of the supercurrent is measured. 
a)

$$
\Psi_{1}=\sqrt{\rho_{1}} \mathrm{e}^{\mathrm{i} \phi_{1}}
$$

$$
\Psi_{2}=\sqrt{\rho_{2}} \mathrm{e}^{\mathrm{i} \phi_{2}}
$$

b)
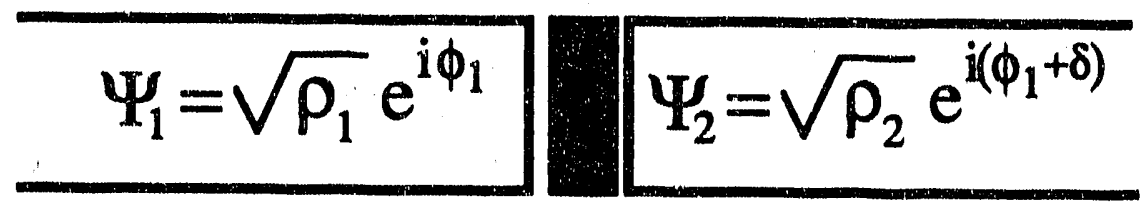

Figure 3.2: a) The electron pairs in a superconductor all have the same phase which is independent of the phase of another distant superconductor. b) When the two superconductors are close enough to allow the electron pairs to tunnel between them, the phases of the electron pairs in the two superconductors become related. This forms a Josephson junction. 
Since a dC SQUID consists of two Josephson junctions in parallel, we need to look at this next $[44,53,54,55,56,57]$. Figure 3.1 shows that the total supercurrent at point $\mathrm{Q}$ consists of the supercurrent through junctions $\alpha$ and $\beta$, resulting in

$$
j_{T}=j_{1 \alpha} \sin \left[\delta_{\alpha}(t)\right]+j_{1 \beta} \sin \left[\delta_{\beta}(t)\right]
$$

where the phases $\delta_{\alpha}(t)$ and $\delta_{\beta}(t)$ are given by Equation 3.2. 'The basis of the SQUID is the fact that an electron pair moved between points 1 and 2 acquires a phase shift from the magnetic field given by

$$
\delta=\frac{-2 e}{\hbar} \int_{1}^{2} \vec{A} \cdot d \vec{s}
$$

where $\vec{A} \cdot d \vec{s}$ is the component of the vector potential along the path traveled by the electron pair. The expression above gives us the phase shift of the pairs traveling through the vector potential inside the superconducting arms of the SQUID. Even though it is well known that a magnetic field cannot penetrate deeply into a superconductor, the magnetic field corresponds to the curl of $\vec{A}$. Thus, a spatially invariant vector potential does not contradict the absence of a magnetic field. This is in fact the basis of the Aharanov-Bohm effect which is closely related to the interference effect of the SQUID [58, 59].

There exists a boundary condition which these superconducting electron pairs must satisfy, being that, upon moving an electron pair through one complete circuit, the phase shift of the wavefunction must be an integer multiple of $2 \pi$. This boundary condition is given by

$$
2 \pi n=\delta_{\alpha}(t)-\delta_{\beta}(t)-\frac{2 e}{\hbar} \oint_{C} \vec{A} \cdot d \vec{s} .
$$

Stokes' Theorem gives

$$
\frac{2 e}{\hbar} \oint_{C} \vec{A} \cdot d \vec{s}=\frac{2 e}{\hbar} \Phi_{L}
$$


where $\Phi_{L}$ is the magnetic flux threading through the SQUID [7]. The flux quantum $\Phi_{0}$ has been defined as $\Phi_{0} \equiv \frac{h}{2 a} \approx 2.07 \times 10^{-7} \mathrm{G} \cdot \mathrm{cm}^{2}$. Setting $n=0$ for simplicity, Equation 3.4 can be rewritten as

$$
j_{T}=j_{1 \alpha} \sin \left[\delta_{\alpha}(t)\right]+j_{1 \beta} \sin \left[\delta_{\alpha}(t)-2 \pi \frac{\Phi_{L}}{\Phi_{0}}\right]
$$

Assuming the Josephson junctions to be identical $\left(j_{1}=j_{1 \alpha}=j_{1 \beta}\right)$, we get

$$
j_{T}=2 j_{1} \sin \left[\delta_{\alpha}(t)-\frac{\pi \Phi_{L}}{\Phi_{0}}\right] \cos \left(\frac{\pi \Phi_{L}}{\Phi_{0}}\right)
$$

Assuming the bias current $\left(j_{B}\right)$ exceeds the maximurn total critical supercurrent possible $\left(2 j_{1}\right)$, the voltage across the SQUID is proportional to the normal current (i.e. the difference between the bias current and the supercurrent),

$$
V(t)=R\left[j_{B}-2 j_{1} \sin \left[\delta_{\alpha}(t)-\frac{\pi \Phi_{L}}{\Phi_{0}}\right] \cos \left(\frac{\pi \Phi_{L}}{\Phi_{0}}\right)\right]
$$

where $R$ is the effective resistance of the SQUID. Since $\delta_{\alpha}(t)$ oscillates at a very high frequency, a narrowband detector will observe only a time-averaged value of $V(t)$, but the time average of $V(t)$ is not simply zero [57]. When one integrates $\delta_{\alpha}(t)$ over one period, using Equation 3.2 for $\delta_{\alpha}(t)$, it yields the time-averaged voltage

$$
\bar{V}=R\left(j_{B}^{2}-\bar{j}_{T}^{2}\right)^{\frac{1}{2}}
$$

where the time-averaged supercurrent is

$$
\bar{\jmath}_{T}=2 j_{1}\left|\cos \left(\frac{\pi \Phi_{L}}{\Phi_{0}}\right)\right|
$$

Figure 3.3 shows $\bar{V}$ as a function of $\Phi_{L}$, the amount of magnetic flux threading through the SQUID. 


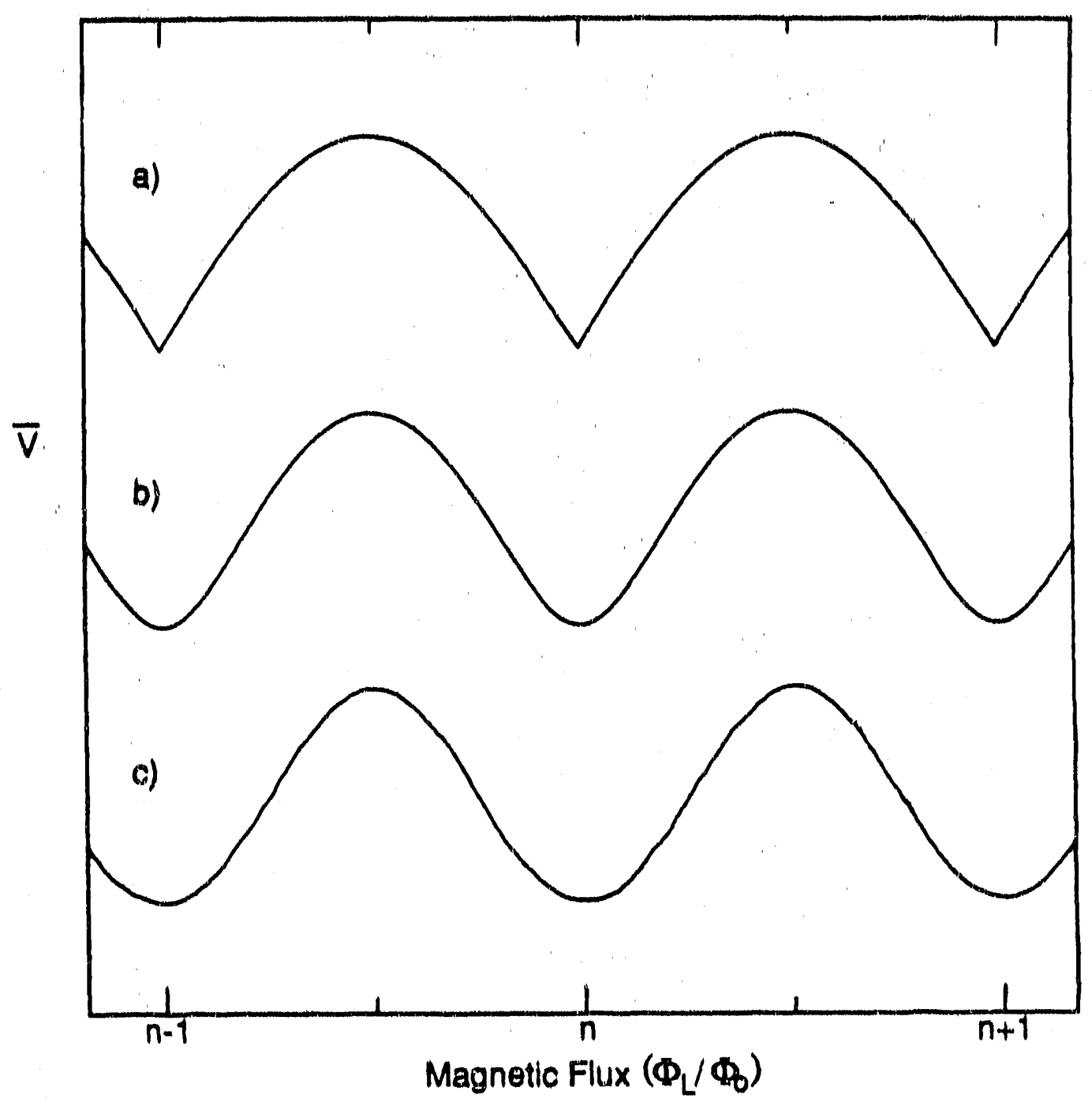

XBL $8911-4293$

Figure 3.3: Time-averaged voltage across a dc SQUID as a function of the magnetic flux through the dc SQUID, using Equations 3.11 and 3.12. a) The bias current is set equal to the maximum supercurrent possible, $j_{B}=2 j_{1}$; and b) $j_{B}=2.12 j_{1}$. c) The experimental flux-voltage curve from our commercial dc SQUID. 
A qualitative way of visualizing how one arrives at Figure 3.3 is to rearrange Equation 3.11 to get

$$
j_{B}=\left[\left(\frac{\nabla}{R}\right)^{2}+j_{T}^{2}\right]^{\frac{1}{2}},
$$

where $j_{B}$ is the blas current applied to the SQUID. This equation is valid only when $\left|j_{B}\right| \geq\left|j_{T}\right|$. When $\left|j_{B}\right|<\left|j_{T}\right|$, the voltage across the SQUID is zero since all of the electron flow is a supercurrent. A plot of the $j_{B}$ versus $V$ curve, for nonnegative voltages, for the dc SQUID is shown in Figure 3.4. Since $\dot{j}_{\mathrm{r}}$, also called the critical current, depends on the amount of flux through the SQUID, we can get curves (a), (b), and (c) depending on the amount of magnetic flux. Typically, one always sets the bias current such that $\left|j_{B}\right|>\left|\vec{j}_{T, \text { max }}\right|$, shown by the horizontal line (d) in Figure 3.4. This shows that when $\Phi_{L}$ is varied, the voltage across the SQUID varies periodically between $V_{a}$ and $V_{c}$, resulting in Figure 3.3. Many effects, such as non-identical junctions, self-induced flux in the SQUID, noise, etc., have not been taken into account here $[20,53,54,56,57]$. The observed curve is thus smoothed to approximate a sine function as shown in the experimental $V$ versus $\Phi_{L}$ curve in Figure 3.3.

\subsection{Coupling Magnetic Flux to the SQUID}

The next problem is coupling the mugnetic flux of the sample to the dc SQUID. There are two common upproaches to this problem, the direct and indirect methm. ods. The direct method is used when one wants to measure quantities such as the earth's magnetic field. The SQUID is placed such that the earth's magnetic field is orthogonal to the plane defined by the SQUID, making the magnetic flux thread through the SQUID [60]. The indirect method is used when one wishes to measure 


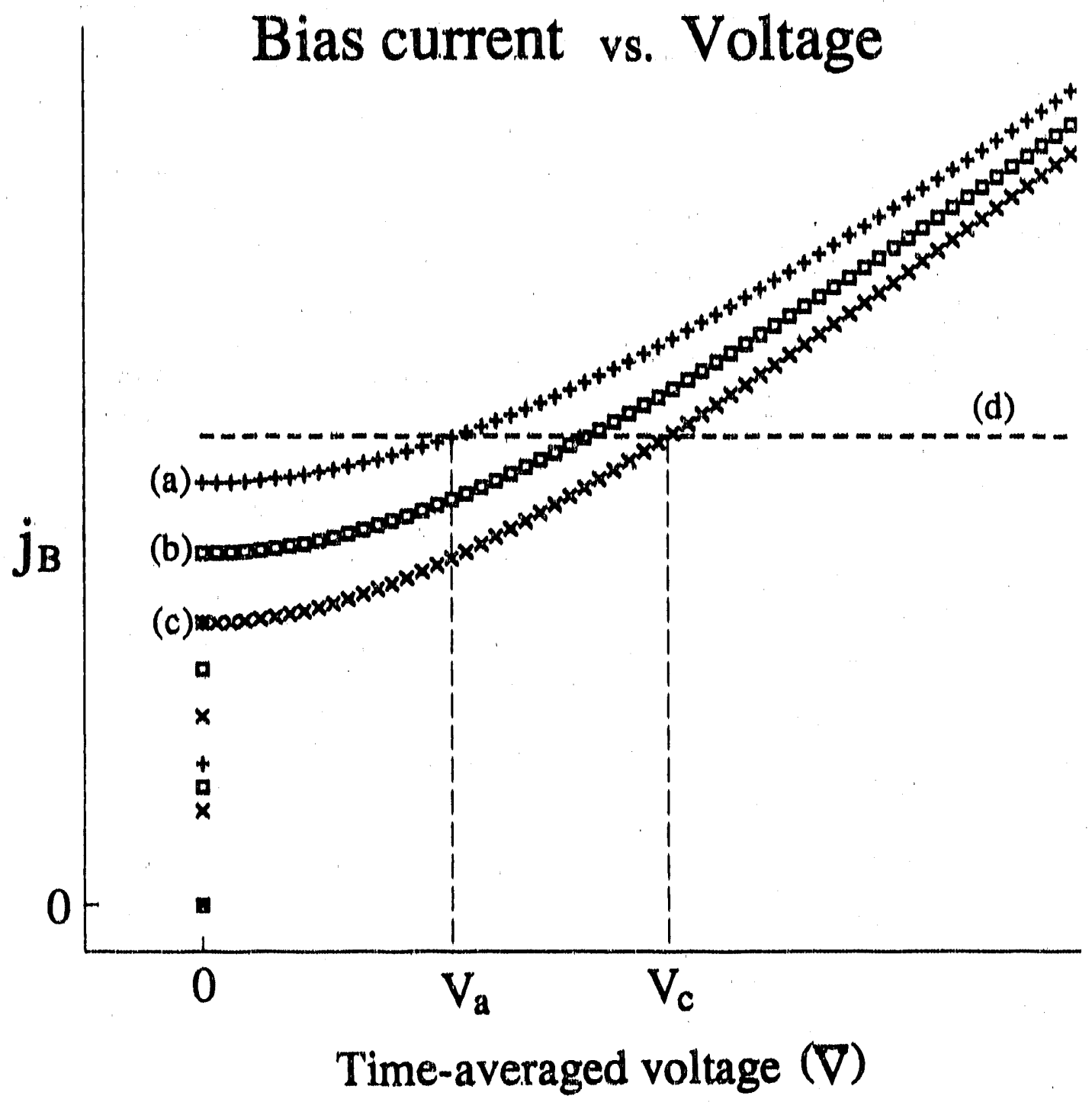

Figure 3.4: A plot of the bias current as a function of the time-averaged voltage across the dc SQUID, for various amounts of magnetic flux through the dc SQUID $\left(\Phi_{L}\right)$. Usually, the bias current $\left(j_{B}\right)$ applied to the dc SQUID is fixed (curve (d)). The voltage across the dc SQUID depends on the flux through the SQUID, and oscillates periodically between $V_{a}\left(\Phi_{L}=n \Phi_{0}\right.$, curve (a)) and $V_{c}\left(\Phi_{L}=\left(n+\frac{1}{2}\right) \Phi_{0}\right.$, curve (c)), depending on the flux through the SQUID. 
the magnetic flux of small samples, as in NMR, NQR, and magnetic susceptibility measurements. In this coupling approach, a magnetic flux transformer is utilized to transfer the flux from the sample to the SQUID [57]. This has the advantages of being able to have the sample at a different temperature than the SQUID or to have the sample in a large magnetic field while keeping the SQUID in a low or zero magnetic field. Although it may be possible to place a sample directly into a SQUID, it would require a very small sample size, and the ability to easily change the sample would degrade the shielding of the SQUID from undesireable magnetic flelds.

In the coupling approach, one could work either with a tuned circuit or a wideband, untuned superconducting circuit. The tuned circuit has the advantage of amplification by a factor on the order of $Q$ (the quality factor of the coil) and the only noise measured is within the bandwidth of the circuit. However, since the tuned circuit still obeys Faraday's Law $\left(V \propto \frac{d \phi}{d t}\right)$, signal intensity decreases as the frequency of the signal decreases. The other disadvantage is that the circuit is usually tuned to a relatively narrow bandwidth $[61,62,63,64]$, making it necessary to extensively retune the circuit and conduct piecewise acquisition to detect over a large frequency range. This makes the tuned circuit ill-suited for wideband, lowfrequency detection.

The best approach for this SQUID NQR technique, to detect low frequency magnetic resonances over a large bandwidth, is to use an untuned superconducting circuit to couple the sample flux to the SQUID. This circuit consists of two superconducting coils connected by superconducting wire, Figure 3.5. The pickup coil encircles the sample while the other, the coupling coil, is tightly coupled to the 


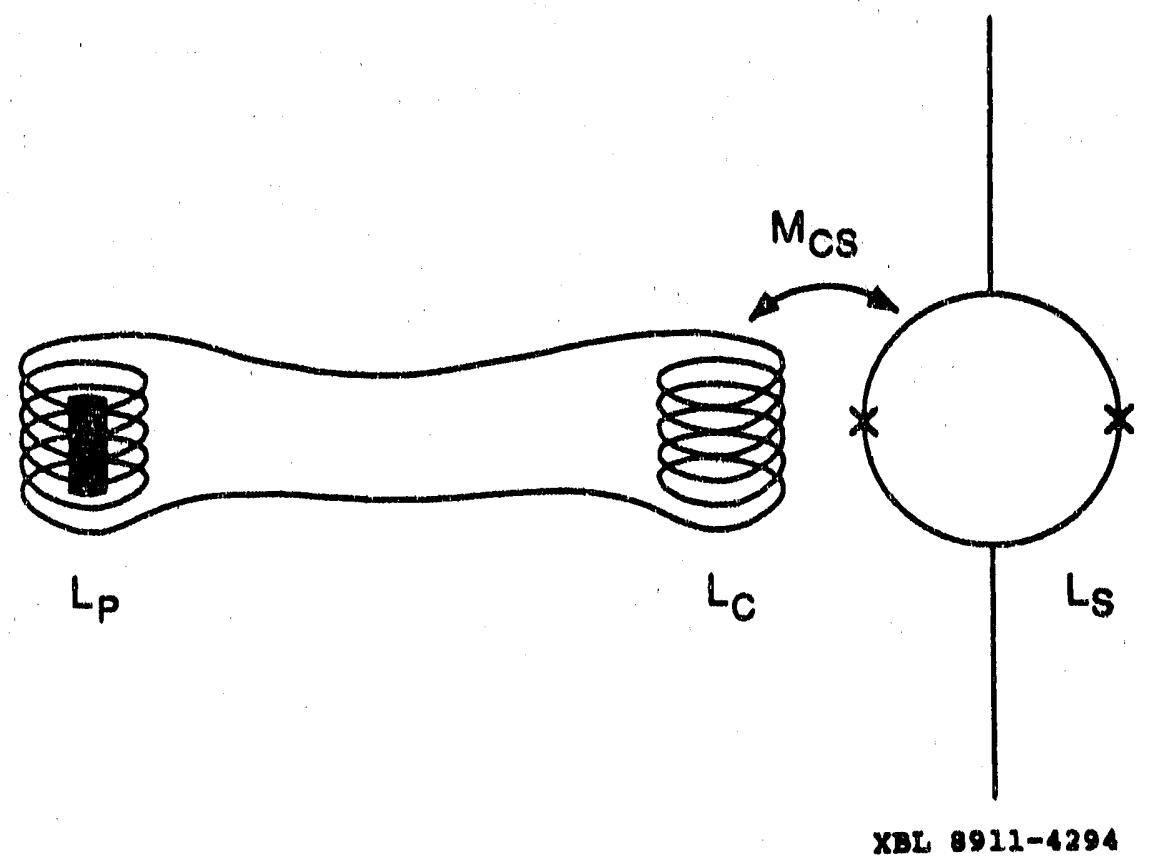

Figure 3.5: A schematic of the superconducting transformer used to transfer the sample flux to the dc SQUID. The pickup coil and the coupling coil form a closed loop and $M_{C S}$ is the mutual inductance between the coupling coil and the dc SQUID. A change in the magnetic flux through the pickup coil produces an opposing change at the coupling coil. This change in magnetic flux is then coupled into the dc SQUID. 
SQUID. The key operating principle behind this circuit is that the total flux enclosed by the closed superconducting loop, the two coils and interconnecting wires, remains constant [50]. When the magnetization of the sample changes and alters the magnetic flux through the pickup coil, a superconducting current is generated in the wire. This superconducting current produces magnetic flux, of equal magnitude but of opposite sign, at the coupling coil to keep the total flux through the circuit constant. This circuit is responsive to signals that have frequencies in the megahertz down to dc frequencies. Since the coupling coil is tightly wound near or around tife SQUID, the SQUID will feel the magnetic flux of the sample. This circuit, as will almost all others, suffers losses and this results in only a fraction of the sample magnetization being transferred to the SQUID. The design of the pickup coil usually plays a large part in the sensitivity of this technique, and thus deserves a more detailed examination. The coupling coil of our commercial SQUID system could not be altered since it was in a hermetically sealed chamber with the dc SQUID.

The magnetic flux generated in the pickup coil by a sample of magnetization $M_{a}$ is

$$
\Phi_{P}=4 \pi f M_{a} \pi r_{P}^{2} N_{P} \quad(\mathrm{cgs})
$$

where $f$ is the filling factor, $r_{P}$ is the radius of the pickup coil, and $N_{P}$ is the number of turns in the pickup coil. This magnetization will induce a currert in the pickup coil of

$$
I_{P}=\frac{\Phi_{P}}{L_{C}+L_{P}}
$$

where $L_{P}$ and $L_{C}$ are the inductances of the pickup and coupling coils respectively. Any stray inductances in the superconducting circuit should be added to the other 
terms in the denominator. The amount of magnetic flux ultimately coupled into the SQUID, sometimes called the input flux, depends on the mutual inductance between the coupling coil and SQUID $\left(M_{C S}\right)$, as given by

$$
\Phi_{L}=I_{P} M_{C S}
$$

or

$$
\Phi_{L}=\frac{M_{C S}}{L_{P}+L_{C}}\left(4 \pi f M_{a} \pi r_{P}^{2} N_{P}\right) .
$$

The objective is to maximize $\Phi_{L}$ for a given $M_{a}$. Previous work has shown that the maximum signal is attained when $L_{P}=L_{C}[47,65]$, when the sample size is fixed. Numerical simulations become necessary when the situation becomes more complex when allowing the sample size to change [66]. The practical aspects of maximizing $\Phi_{L}$ are presented in Chapter 4.

\subsection{Output Voltage of the SQUID System}

The following provides a qualitative description of the lock-in detection and feedback techniques used to be able to generate a large signal from the SQUID $[45,47$, 67]. A brief discussion on electromagnetic inteference effects on the SQUID then follows.

One generally designs the SQUID system to be able to measure, with high sensitivity, low frequency signals down to dc signals. The obstacle in measuring very low frequency signals is that the system's noise power, which is white at higher frequencies, is $\frac{1}{f}$ noise at very low frequencies [47]. Since very low frequency measurements are often desired, one wishes to reduce the $\frac{1}{f}$ noise contribution which dominates at frequencies below about a hertz. The usual remedy to $\frac{1}{f}$ noise is to 
use signal modulation, better known as lock-in detection [68]. The lock-in detection technique circumvents various sources of $\frac{1}{f}$ noise in the system such as drifts in the bias current and changes in the critical current due to small changes in temperature.

Figure 3.6 shows a schematic of the SQUID along with the coupling coil, modulation coil, lock-in detection electronics, and feedback mechanism. The coupling and modulation coils are both tightly flux-coupled to the SQUID. The principle of signal modulation, as it applies to the SQUID, is to use the modulation coil to apply an additional flux $\left(\Phi_{m}\right)$ of frequency $\nu_{m}$ and of about half a flux quantum peak-to-peak in amplitude. The resulting oscillating voltage across the SQUID will, in general, consist of a component at $\nu_{m}$ and another at $2 \nu_{m}$, Figure 3.7. The peak-to-peak amplitude of each component will depend on exactly where one is on the original voltage versus flux curve, Figure 3.3. If the average flux through the SQUID is $\left(n+\frac{1}{4}\right) \Phi_{0}$, the $\nu_{m}$ component will be maximized and the $2 \nu_{m}$ component minimized. If the average flux through the SQUID is $\left(n+\frac{1}{2}\right) \Phi_{0}$, the $\nu_{m}$ component will be minimized and the $2 \nu_{m}$ component maximized. When the average flux is $\left(n+\frac{3}{4}\right) \Phi_{0}$, it will be the same as when the flux is $\left(n+\frac{1}{4}\right) \Phi_{0}$ except the $\nu_{m}$ component will be phase shifted by $180^{\circ}$ with respect to the reference modulation signal. With the use of a phase sensitive detector [68], it becomes clear that the amplitude of the $\nu_{m}$ component is a direct measure of the sample flux. The modulated signal from the SQUID is then connected to a circuit tuned to the frequency $\nu_{m}$. This results in a signal, multiplied approximately by the $Q$ of the circuit, which is then mixed with the reference signal to give a lock-in output of near dc or dc frequency. The dc output voltage of the lock-in detector with respect to the input flux can be qualitatively described as $V_{\text {output }} \sim \sin \left(\frac{2 \pi \Phi_{L}}{\Phi_{0}}\right)$. There are higher-order harmonics 


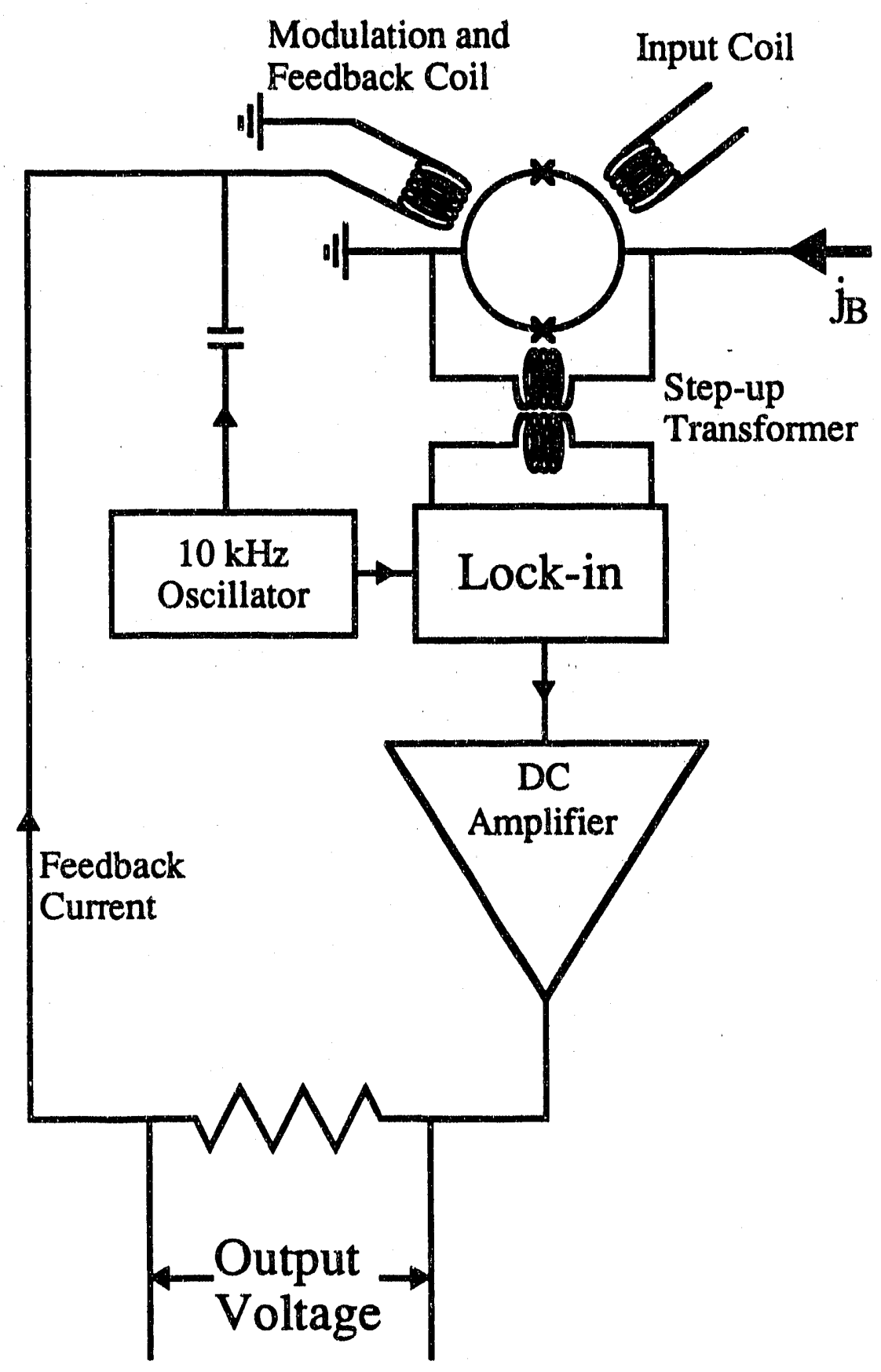

Figure 3.6: A schematic of the SQUID system, along with the coils, lock-in electronics, and feedback mechanism. This arrangement allows for low system noise, high sensitivity, and a large dynamic range. 

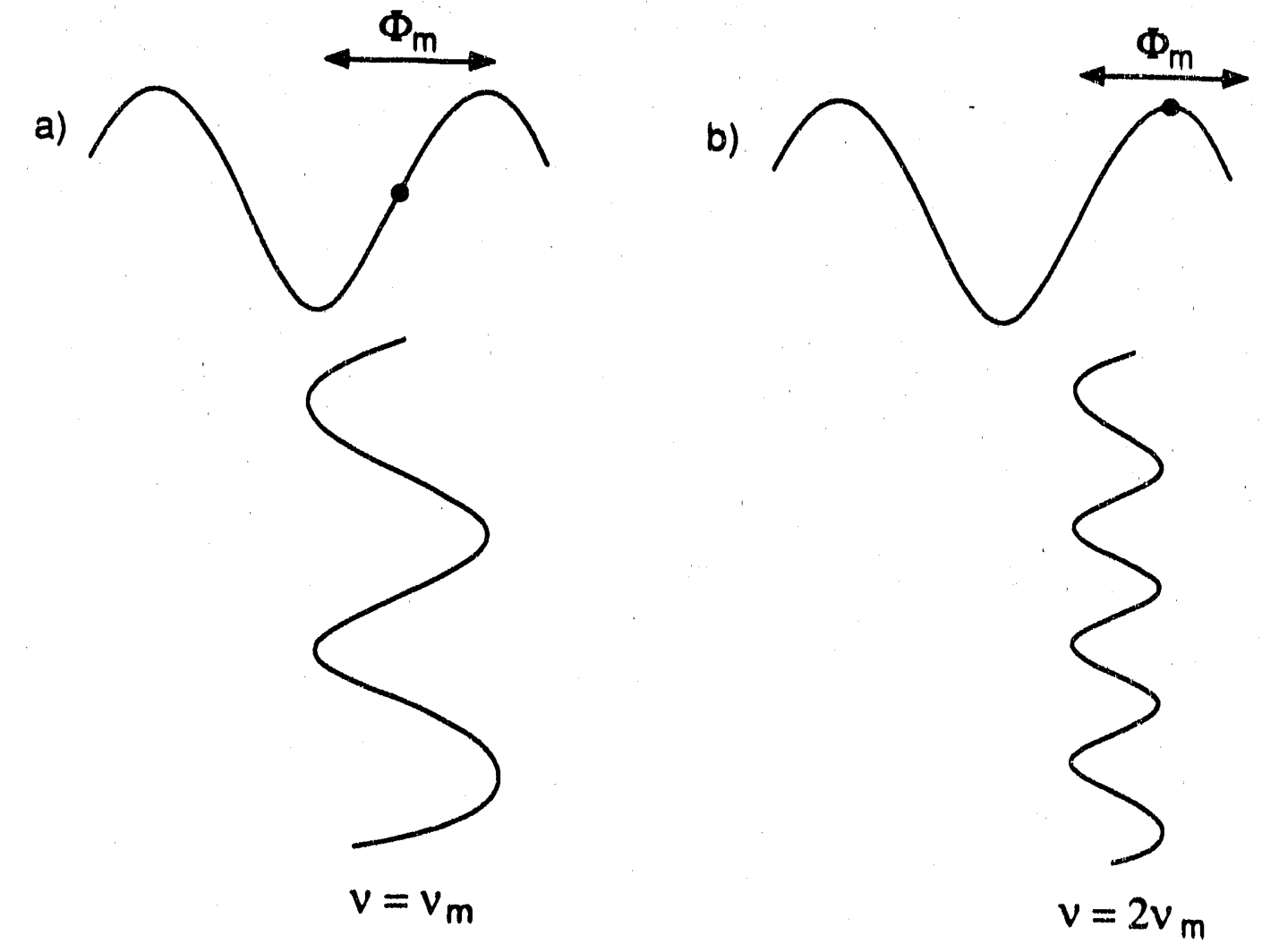

XBL $8921-4295$

Figure 3.7: Signal modulation and lock-in detection scheme: The total magnetic flux through the dc SQUID is the sum of the flux transferred from the sample $\left(\Phi_{L}\right)$ and a weak, oscillating modulation flux $\left(\Phi_{m}=\frac{\Phi_{0}}{2} \sin \left(\nu_{m}\right)\right)$. a) $\Phi_{L}=\left(n+\frac{1}{4}\right) \Phi_{0}$, the voltage across the dc SQUID oscillates at a frequency $\nu_{m}$ and there is no component at $2 \nu_{m}$. b) $\Phi_{L}=\left(n+\frac{1}{2}\right) \Phi_{0}$, the modulation causes the voltage to oscillate at $2 \nu_{m}$ and no component at $\nu_{m}$ is present. Thus, the amplitude of the voltage oscillating at $\nu_{m}$, along with the use of a phase sensitive detector, allows us to know where one is on the flux-voltage curve. 
of $\nu_{m}$ involved in all of this but they are not significant to this discussion.

In most cases, one desires a SQUID system and lock-in detection which provides a linear output voltage response to any size input flux, especially very large signals. As it stands now, the output voltage of the lock-in device is qualitatively a periodic function of the sample flux as shown in Figure 3.8. This means that the output voltage of the lock-in detector is proportional to the input flux only when the sample generates much less than one flux quantum in the SQUID. Negative feedback is used with the SQUID and lock-in detection to increase the linear dynamic range of the system [69]. Negative feedback consists of inverting the output of the lock-in, amplifying it, and feeding it back to the SQUID as magnetic flux, thus opposing the input flux. This feedback flux cancels the input flux at the SQUID and holds the total flux through the SQUID at some constant value. The voltage used as the feedback flux is also applied through the modulation coil and is shown as $V_{\text {output }}$ in Figure 3.6. $V_{\text {output }}$ is now linearly proportional to the input flux and sample flux, no matter its size (within limits), and the average total flux through the SQUID remains fixed, making it a null detector. In addition to the output voltage of the SQUID system ( $V_{\text {output }}$ ) having a linear response to even very large inputs, it also is relatively independent of the exact shape of the periodic voltage versus flux curve.

It may be of interest to point out some features of this technique at this point. Should the sample flux oscillate at $\nu_{s}$, the voltage after signal modulation oscillates at $\nu_{m} \pm \nu_{s}$. Lock-in detection and mixing down recovers the component at $\nu_{s}$. This means that the tuned circuit and lock-in detector must have a low $Q$ in order to measure signals oscillating at a significant fraction of the modulation frequency. This also means that signals oscillating at frequencies higher than the modulation 


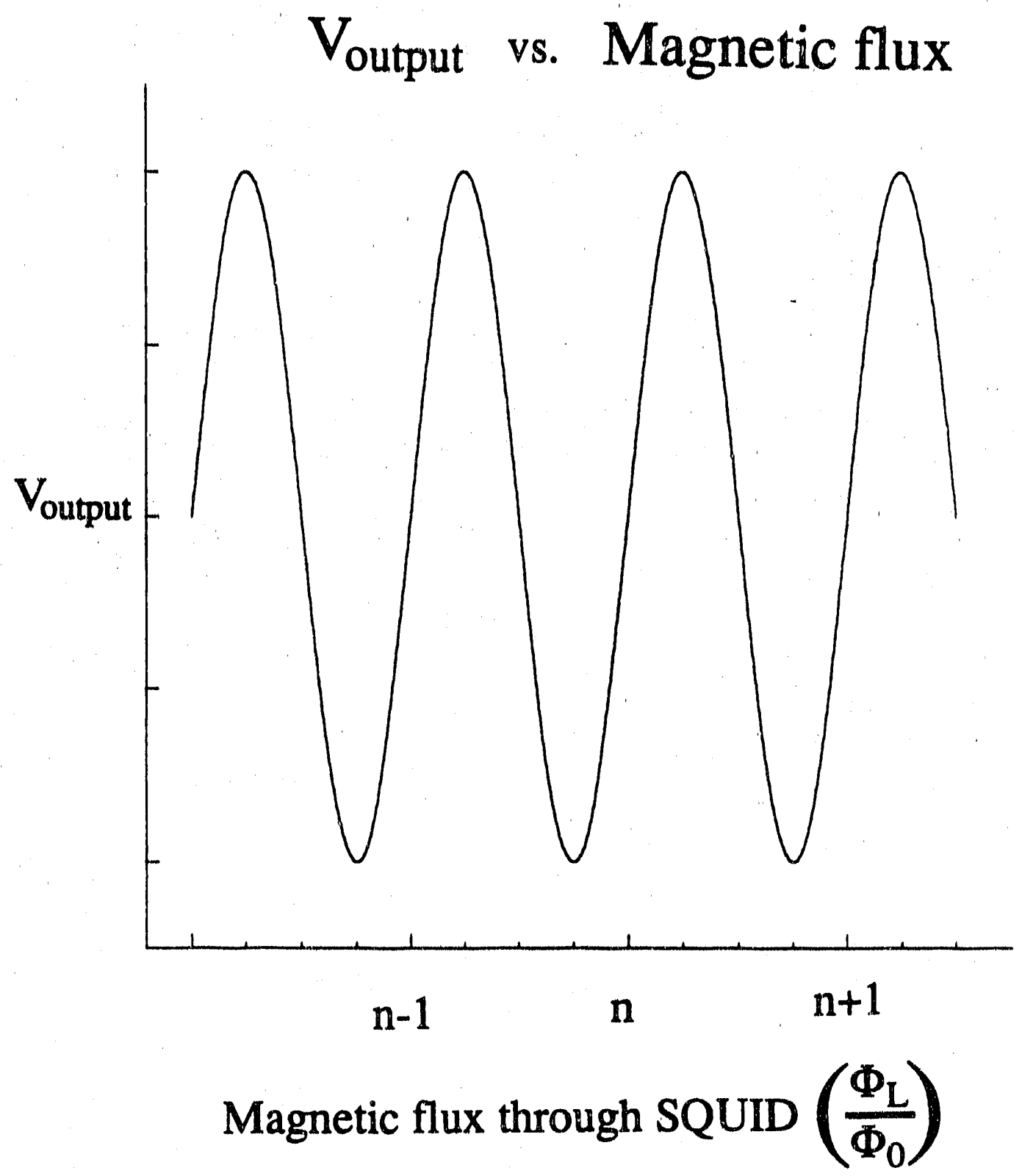

Figure 3.8: Final output voltage versus flux curve of a dc SQUID system when signal modulation and lock-in detection are taken into account. 
frequency $\left(\nu_{m}\right)$ cannot be detected with this modulation and feedback arrangement. The modulation frequency is limited by unavoidable stray reactances in the detection and feedback circuits and are generally lirnited to a few hundred kilohertz. This limits the use of these flux-locked SQUID systems to relatively low frequency pulsed NMR and NQR measurements. This frequency limitation is, however, of no real concern when using z-axis magnetic resonance as described in Chapter 2. Another point pertains to exactly where on the voltage versus flux curve one usually holds the average total flux through the SQUID constant. In order to achieve a linear response from the SQUID system, one wishes to operate where the output voltage versus flux curve is linear. Figure 3.8 tells us that two points satisfy this requirement, $\Phi_{L}=n \Phi_{0}$ and $\Phi_{L}=\left(n+\frac{1}{2}\right) \Phi_{0}$. Usually, the latter point is chosen. The reader is referred to further readings for the particular reason $[20,45,47]$.

As is evident from the preceeding discussion, the SQUID is very sensitive to electromagnetic interference since a flux quantum $\left(\Phi_{0} \approx 2.07 \times 10^{-7} \mathrm{G} \cdot \mathrm{cm}^{2}\right)$ is very small. Two types of interference with high freciuencies involved are of most concern: low-level interference and large, sharp transients. Since the low-level high frequency interference has a frequency greater than the modulation frequency, it modulates the voltage versus flux curve. The net effect is to reduce the peak-topeak amplitude of this curve and reduce the slope of Figure 3.8 at $\Phi_{L}=\left(n+\frac{1}{2}\right) \Phi_{0}$. This reduces the signal-to-noise ratio for a given signal size. Large, sharp transient interference contains high frequencies in order to produce the fast rises and edges of the interference [70]. Should these reach the SQUID, the flux in the SQUID will change faster than the feedback circuit can follow. This causes the system to "lose lock" and when it does lock-in again, the new point will be different from the 
original point by an integer number of flux quanta. The dc output voltage will, thus, jump from one value to another. These voltage jumps will be integer multiples of the dc offset corresponding to one flux quantum through the SQUID. These effects make it prudent to shield the SQUID and its inputs from any interference with frequencies higher than the modulation frequency. The practical aspects of eletromagnetic interference shielding are addressed in Chapter 4. 


\section{Chapter 4}

\section{SQUID NQR Spectrometer}

This chapter describes the low-temperature probe and spectrometer which was built around the commercial dc SQUID system. We first take a look at some considerations when designing these parts and then examine some aspects in greater detail. The performance of the entire spectrometer is examined at the end of the chapter.

\subsection{Probe aud Spectrometer Design Considera- tions}

In theory, the experimentalist usually wishes to maximize the sensitivity of the experimental apparatus. In practice, this is not always possible when other experimental factors have to be considered and accounted for. These factors include the nature of the materials used in construction, optimum design of the pickup coil, and shielding of the system from unwanted electromagnetic radiation. Other, more practically pertinent, factors are the ease of assembly and disassembly (or adjustments) to the probe and the ability to easily change the sample. The reader is asked 
to bear in mind that we had no extensive previous experience with low-ternperature apparatuses and that this is a first-generation plece of equipment. There is no doubt that improvements can be made to the design and construction of this equipment, and to enhance its sensitivity.

The low-temperature probe, being a frst-generation apparatus, was designed and constructed with ease of assembly and disassembly in mind. This was to allow the change of its configuration without having to rebuild many parts repeatedly, and to be able to slightly alter the relative orientations of coils and determine its effects. However, this type of design makes for an inherently less rigid structure than a permanent one. A rigid structure is important because the pickup coll is usually located in a region of large magnetic field, many orders of magnitude greater than the magnetic field of the sample. When microscopic movements or vibrations occur, this makes the pickup coil move in an inhomogenous magnetic fleld, chang. ing the amount of flux through the pickup coil thereby increasing the noise level of the system. Improving the homogeneity of the magnetic field helps, but one is still required to maintain structural rigidity. Previously, ease of assembly and disassembly of the coil structure was not designed into SQUID spectrometers because of the above problem of relative motion between the pickup coil and magnetic flelds. Many of those previous designs cast the entire coil structure, consisting of pickup coil, dc field coils, and rf coils, in permanent configurations using epoxy $[71,72,73]$. This did not permit small adjustments or different geometries of the pickup coil to be tested without extensive remachining of coils. We believe that we have reached a reasonable compromise between the rigidity of the coil structure and the ease of its assembly and disassembly. 
The highest sensitivity can be obtained when the pickup coil is wrapped directly around the sample. This, however, makes it difflcult to change the sample without taking the probe out of the low-temperature bath which is to be avolded because the large thermal mass of the probe will greatly increase the boil-off of cryogens when it is reinserted into the bath. The best solution is a design that allows for changing the sample while keeping the probe in the low-temperature bath. Doing so also allows for the possibility of using another lock-in detection scheme where one rapidly cycles the sample in and out of the pickup coil during signal acquisition.

If the temperature of the sample could be varied, while keeping the SQUID and colls at the bath temperature, the versatility of the SQUID spectrometer would be vastly increased. This would require a separate dewar, to hold the sample and maintain its temperature, to fit inside the pickup coil. A common design, which has been used in SQUID NMR $[24,74,75]$ and SQUID magnetic susceptibillty measurements $[71,76,77]$, is to use a reentrant dewar inside the pickup coil. There are two negative aspects to a variable-temperature probe. The first is that the filling factor of the pickup coil would be reduced, resulting in a reduction of signalto-noise. Secondly, the signal intensity at , say, liquid nitrogen temperature would be reduced by a factor of approximately 20 compared to the signal intensity at liquid helium. This is due to the smaller population difference, given by the Boltzmann distribution, at liquid nitrogen temperature.

The nature and magnetic behavior of the materials used to build the probe, especially near the pickup coil, is also of considerable importance. Materials which have magnetic resonance signals that can be confused with sample signals should be avoided. This includes materials which generate noise that affect or can be 
detected by the SQUID. Day and others found numerous materials possess temperature dependent electron paramagnetism in magnetic fields $[76,78]$. The magnetic flux produced by these materials could couple into the pickup coil, resulting in an increase in the noise in the output of the SQUID, especially when one considers that small temperature variations cause variations in the amount of flux produced by these materials. It is also advisable to keep superconducting materials out of the pickup coil since the supercurrents generated to counter rf radiation are generally in fluctuating directions. This means that materials used for superconducting magnetic shielding, such as lead, should be used only at relatively large distances from the pickup coil. Along with these considerations on construction materials, one should also keep in mind that it is important to hold the probe temperature as stable as possible and to choose materials which do not transfer heat from the outside efficiently, so as to reduce the boil-off rate of expensive cryogens.

An important consideration in the design of the pickup coil is its type, single solenoid or gradiometer coils [21], and its geometry. If one assumes that the mutual inductance between the coupling coil and the SQUID is fixed, as is in many commercial SQUID systems, then all the pickup coil parameters may be variables. The diameter of the superconducting wire was not considered to be a variable due to the availability of very high quality wire of reasonable diameter. When deciding whether to use a gradiometer pickup coil or a solenoid pickup coil, one must consider the following. The gradiometer coil consists of two nearly identical coils, wound in opposite directions, placed in series. The sample is placed in one of the coils and the other is left vacant. The advantage of the gradiometer coil design is that in the presence of large scale magnetic fluctuations, such as fluctuations in the homoge- 
neous dc magnetic field applied, it can attenuate these fluctuations by two to three orders of magnitude. The disadvantages of the gradiometer are a restriction on the length of the sample and a reduction in the flux transfer efficiency from the sample to the SQUID. The second disadvantage comes about from the matching condition of the pickup coil and the coupling coil, i.e. $L_{P}=L_{C}$. This condition necessitates that the number of turns in each half of the gradiometer be reduced by a factor of $\frac{1}{\sqrt{2}}$ from the number of turns possible in a single solenoid coil. So maximum flux sensitivity is obtained with a single solenoid coil, provided large scale fluctuations are minimized.

When optimizing the geometry of the pickup coil, one wishes to maximize the flux through the SQUID with respect to the flux of the sample. Assuming the mutual inductance between the coupling coil and SQUID is fixed, previous work has shown that maximum flux transfer occurs when $L_{P}=L_{C}$ [65]. The one independent variable left is the radius of the pickup coil $\left(r_{P}\right)[78,79]$. Since $L_{P} \propto r_{P} N_{P}^{2}$, the flux through the SQUID will increase with a. $r^{1}$ dependence for a given sample magnetization. So a larger signal will be observed with a larger radius pickup coil, provided the filling factor of the coil remains relatively constant. The larger pickup coil has the added benefit of a slightly better filling factor since a larger fraction of the area of the coil is occupied by the sample and less by the sample container. However, there are practical considerations when designing the pickup coil. Larger pickup coils require larger $\mathrm{dc}$ and $\mathrm{rf}$ coils, along with more power, to be able to generate dc and rf magnetic fields of given strengths. Larger pickup coils would also necessitate the use of larger volurnes of samples which may be expensive, scarce, toxic, or highly reactive. There are always physical constraints, such as the 
size of the dewar, which may place a limit on the pickup coil geometry. This is so because any magnetic shielding used may start to distort the flux lines, forcing some of them to return through the pickup coil. Calculations have been made by Osterman and Williamson describing this effect.

A difficuli problem in designing the spectrometer was how to shield the SQUID system from unwanted magnetic interference. This discussion is restricted to the problem when operating in continuous wave mode and we can take a look at the magnitude of this problem. A typical CW magnetic resonance experiment requires rf levels of about $10 \mathrm{mG}$ or more. If all of this field were coupled into a pickup coil of 10 turns, $0.5 \mathrm{~cm}$ in diameter, it would generate about $5 \times 10^{5} \Phi_{0}$ through the pickup coil $\left(1 \Phi_{0}=2.07 \times 10^{-7} \mathrm{G} \cdot \mathrm{cm}^{2}\right) \cdot 10^{4} \Phi_{0}$ would couple into the SQUID if we assume $2 \%$ transfer efficiency to the SQUID. In Chapter 3, it was pointed out that high frequency magnetic fluctuations greater than $\frac{\Phi_{0}}{2}$ would eliminate the gain of the SQUID completely and that low amplitude fluctuations result in increased noise levels. Thus, we need to be able to attenuate the rf fields by about 5 orders of magnitude. An attenuation factor of 100 to 1000 can be obtained by orienting the pickup coil orthogonally to the rf coils. The rest of the attenuation must come from either additionally shielding the pickup coil to prevent coupling to the rf coil or using a filter in the pickup circuit to keep rf from entering the SQUID.

The simplest form of a rf shield is a highly conductive cylinder placed between the pickup and rf coils [39]. The rf coils are placed inside the shield while the pickup coil is placed around the outside of the shield. This usually results in a very poor filling factor of the pickup coil and there are variations in the shield's attenuation factor depending on the frequency of the rf radiation as shown below. The shield 
has a characteristic skin depth, $\delta$, related to its electrical conductivity, $\rho$, by

$$
\delta=\left[\frac{4 \pi}{\mu_{0} \nu \rho}\right]^{\frac{1}{2}} .
$$

An electromagnetic wave of frequency $\nu$ will be attenuated, by the shield, by a factor $e^{-\frac{t}{\delta}}$ where $t$ is the thickness of the shield. This allows the very low frequency sample signal to be detected by the pickup coil and still attenuates the rf radiation felt by the pickup coil. However, one can not just minimize the cutoff frequency of the shield. This is because the shield has some resistance $(R)$ to currents encircling it and therefore generates Johnson noise. The amount of noise generated by the shield is inversely related to the shield's lower cutoff frequency. The Johnson noise voltage recorded with a voltmeter of bandwidth $B$ is

$$
V_{n}=(4 k T R B)^{\frac{1}{2}}
$$

where $T$ is the temperature of the shield. Since we are concerned with current noise which produces flux noise, $I_{n}=\frac{V_{n}}{R}$, the flux noise increases as the resistance of the shield is lowered. A thicker shield, reducing the cutoff frequency of the shield, has lower resistance and thus higher flux noise given by $\Phi_{n}=L_{S} I_{n}$ where $L_{S}$ is the inductance of the shield. Alternatively, it is possible to enclose the pickup coil in a shield which wraps around the outside of the sample, leaving the rf coils furthest from the sample. However, this design tends to distort the intensity and homogeneity of the rf fields at the sample.

The second method of shielding the SQUID from rf interference using a filter does not cause a poor filling factor, but still exhibits some generation of flux noise. The rf filter is formed by placing a small shunt resistor $\left(R_{s}\right)$ in parallel with the 
coupling coil $[73,80]$, Figure 4.1 . This $R L$ filter has a low-frequency cutoff of

$$
\nu=\frac{R_{s}}{2 \pi}\left(\frac{L_{P}+L_{C}}{L_{P} L_{C}}\right) .
$$

The key to this filter is that the impedance of the resistor is fixed at $R_{s}$, while the impedance of the coupling coil $\left(\omega L_{C}\right)$ increases with frequency. This allows low frequency currents to pass through the coupling coil, while high frequency currents (frequencies much higher than the filter cutoff frequency) pass through the resistor. Although this filter does produce flux noise, more complex filters have been studied and may prove useful $[22,81]$.

Both types of filters, rf shield and shunt resistor, reduce the transmitted current by $6 \mathrm{~dB}$ per octave at frequencies well above their cutoff frequency. Since they both produce flux noise, a compromise must be reached such that the cutoff frequency effectively reduces $\mathrm{rf}$ interference without generating excessive amounts of noise. This can be done by constructing the probe such that the cutoff frequency is adjustable from the outside of the dewar.

\subsection{Overview of the Spectrometer}

Figure 4.2 is a schematic of the spectrometer. A Faraday cage, made of copper mesh, shields the SQUID and low-temperature probe from high-frequency interference. The SQUID feedback unit is inside the cage while all other electronics is outside. The output voltage of the SQUID control unit passes through a low-pass filter before it is passed on to the chart recorder and/or the computerized data acquisition unit. The rf source is a two channnel synthesizer, one channel of which can also connect to the frequency counter. 


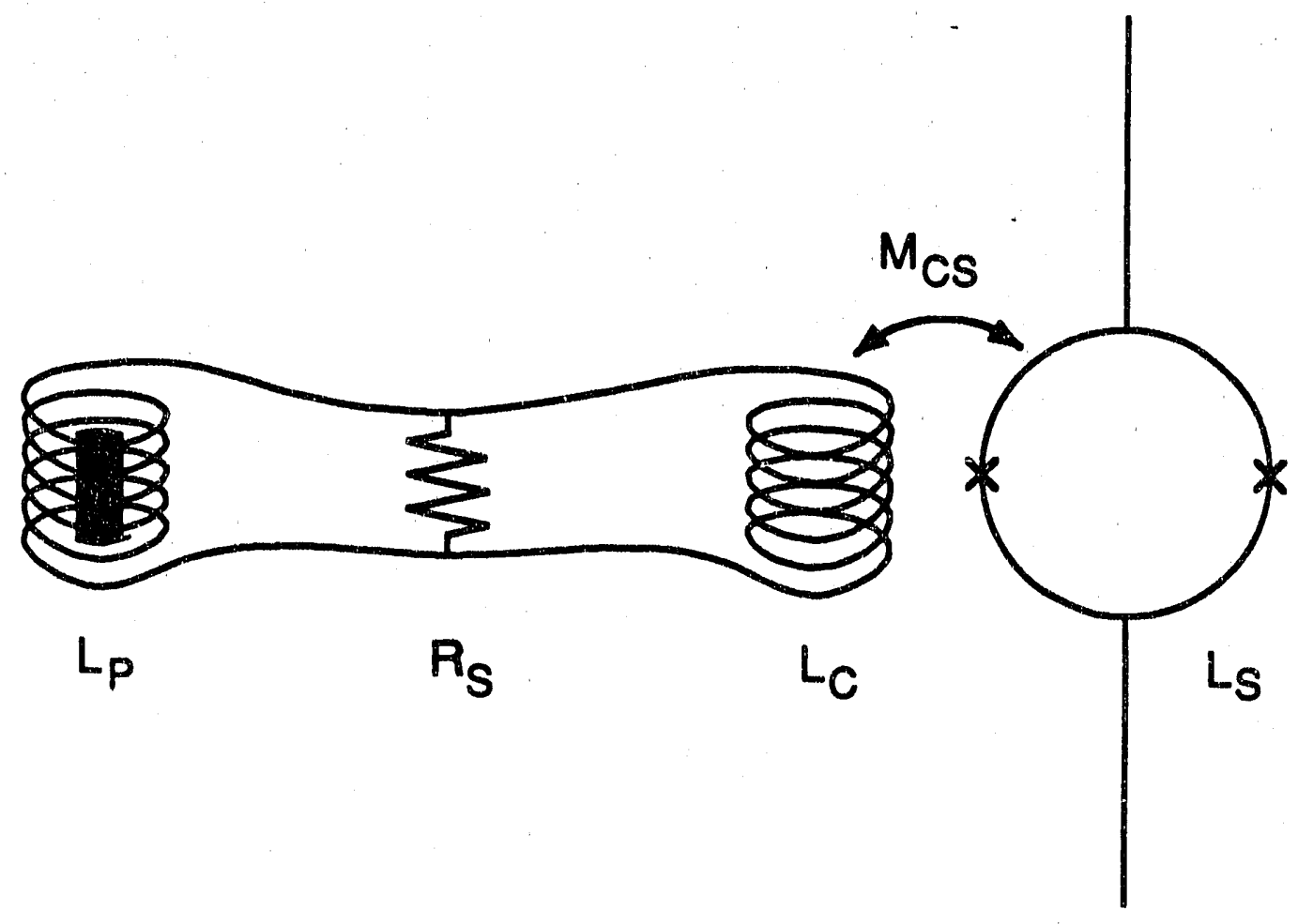

XBZ $8911-3992$

Figure 4.1: Schematic of the shunt resistor $\left(R_{s}\right)$ incorporated into the superconducting flux transformer. This arrangement acts as a low-pass filter, letting low-frequency signals reach the coupling coil while high-frequency interference passes through the resistor instead. 


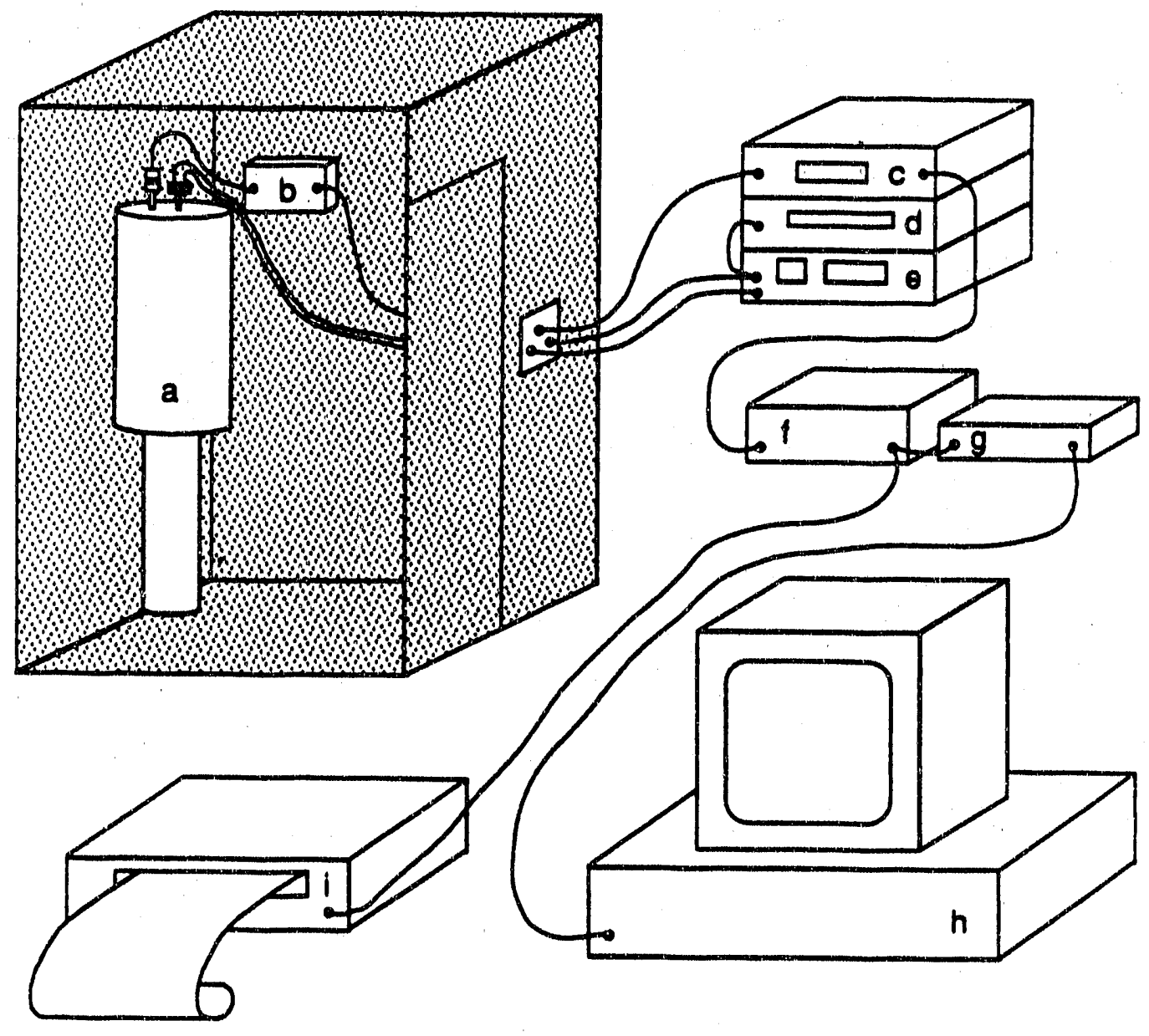

XaL $8921-4296$

Figure 4.2: Schematic overview of the dc SQUID spectrometer. The components are (a)liquid helium cryostat, (b)SQUID feedback unit, (c)SQUID control unit, (d)frequency counter, (e)two-channel rf synthesizer, (f)low-pass filter, (g) analog-to-digital converter, (h)computer, and (i)chart recorder. 


\subsection{Low-Temperature Probe}

The cryostat (dewar) used in this work was originally designed to fit into the bore of a commercial superconducting magnet, resulting in a long and narrow conflguration. The upper half of the dewar has an inside diameter of 3.25 ", necessitating a rather slim probe to fit into the dewar. This imposed additional restrictions when designing the geometry of the entire low-temperature probe.

Figure 4.3 shows a cross-section of the essential parts of the probe, the dc SQUID [82], sample chamber, and shunt resistor board. Most of the exterior of the lower portion of the probe is made of brass, with the exception of the copper cover used to protect the shunt resistor board. The thick brass plates used at the top and bottom of the sample chamber were coated on the inside with solder $(60 \% \mathrm{~Pb}, 40 \%$ $\mathrm{Sn}$ ), while $0.005 "$ thick lead foil was used to line the insides of the sample chamber and the shunt resistor board cover. Solder and lead become superconducting at liquid helium temperature, providing an excellent shield against unwanted electromagnetic radiation at all frequencies. During design and construction of the probe, careful attention was paid to minimizing vibrations within the probe and shielding it from unwanted electromagnetic interference.

The dc SQUID and its associated coils are located in a cannister on the left hand side of Figure 4.3. A niobium shield can be slid off the cannister to expose two 080 brass screws which connect to the coupling coil of the SQUID. The cannister, containing the SQUID and its coils, is permanently hermetically sealed. This was done by the manufacturer to prolong the lifetime of the SQUID. A $\frac{1}{4}-20$ threaded lead nut on the bottom of the cannister secures it to the rest of the probe. The rest of the connections to the SQUID, and its coils, run through a $\frac{1}{4}$ " diameter stainless 


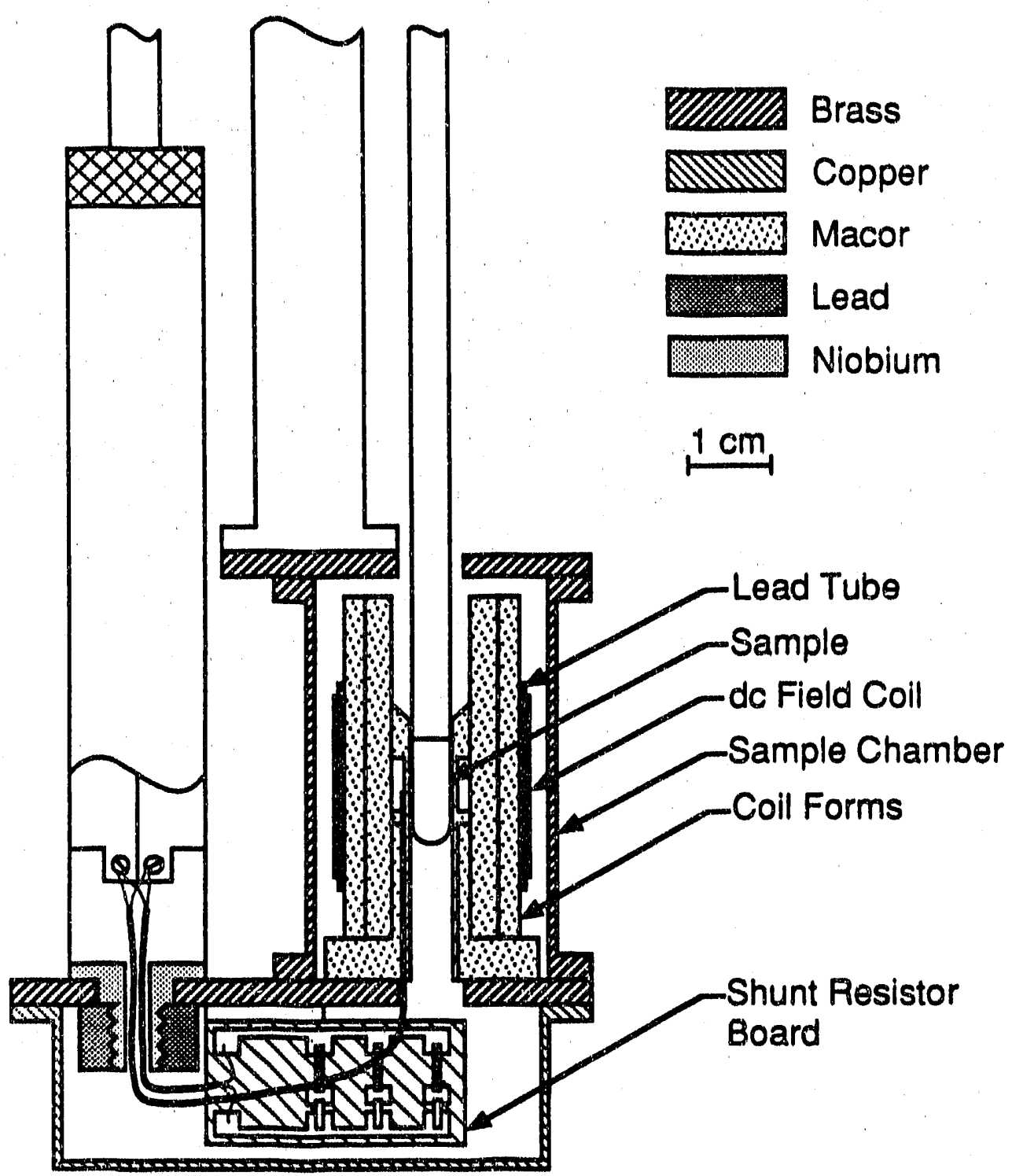

XBL 896-2466

Figure 4.3: Cross-section of the low-temperature probe of the SQUID spectrometer. The dc SQUID is on the left hand side. 
steel tube to the top of the probe. This tube has a fixed length of 31 " and has a 13-pin locking Bendix connector at the top for the cable connection to the SQUID feedback unit.

The coil forms used for the pickup coil, rf coils, and dc field tube were machined from MACOR, a mica glass-ceramic [83]. The pickup coil had an outside diameter of $6.5 \mathrm{~mm}$ and an inside diameter of a few thousandths of an inch over $5.0 \mathrm{~mm}$. A slot about 0.012 " wide and 0.065 " deep was cut around the pickup coil form. The pickup coil was made by tightly winding 10 turns ( 2 rows, each 5 layers deep) of 0.005 " diameter Formvar-insulated NbTi wire into the slot. Cyanoacrylate glue was then applied to the wire in the slot to keep the pickup coil rigid at all times. The leads of the pickup coil were shielded by a $1 \mathrm{~mm}$ o.d. $60 / 40$ lead-tin tube, from the pickup coil form to the coupling coil connections.

The rf coil form fit around the pickup coil form. $\frac{1}{32} " \times \frac{1}{32}$ " slots were machined into the rf coil form such that the dimensions of each Helmholtz saddle rf coils were $26 \mathrm{~mm}$ in length and $16 \mathrm{~mm}$ in diameter, and each formed a $120^{\circ}$ arc to maximize the rf field homogeneity. The two sets of Helmholtz saddle coils were orthogonal, wound with 32 AWG insulated copper wire, and then glued permanently in place with epoxy. The calculated field to current ratio for each set of coils was $3 \mathrm{G} / \mathrm{A}$.

The dc magnetic field coil form was placed around the rf coil form. A lead tube was formed on the outside of the coil form by rolling up and soldering closed a 1 $\mathrm{mm}$ thick sheet of lead. Then a solenoid, 67 turns of 28 AWG insulated copper wire, was tightly wound outside this lead tube. The lead tube and copper solenoid were both glued to the coil form with epoxy. The dc magnetic field was produced by applying a current to the solenoid during the cool down of the probe. When 
the cool down was complete, this current was slowly reduced to zero, producing a trapped magnetic field sustained by superconducting currents in the lead tube [50]. The calculated field-producing efficiency of this arrangement was $30 \mathrm{G} / \mathrm{A}$ and experimental measurements yield approximately $28 \mathrm{G} / \mathrm{A}$. A more homogenous dc field could have been obtained by pouring molten lead into a mold and then letting it slowly cool, to yield a lead tube.

The three coil forms were machined to tolerances of 0.003 " and fit very tightly together. Centering of the coils with respect to each other was to within 0.005 ", and they were centered longitudinally and laterally within the sample chamber to about 0.040 ". To allow easy disassembly or adjustment of the coil forms at room temperature, and still minimize vibrations at low temperatures, the mating surfaces of the coil forms were coated with a thin layer of Apiezon vacuum grease. Two 632 nylon screws were used to securely fasten the entire coil form structure to the bottom of the sample chamber. A $6 \mathrm{~mm}$ diameter hole was drilled through the top brass plate to allow for easy changing of samples. It is doubtful that this degraded the shielding of the pickup coil to a large degree.

The shunt resistor board was made by removing part of the copper conductor from a piece of fiberglass printed circuit board. Three shunt resistors were placed in parallel, also in parallel to the pickup coil, across the inputs to the coupling coil of the SQUID. These resistors, 1 to $0.1 \Omega$, were made from strands of 0.005 " diameter manganin wire which has a room temperature resistance of $11.67 \Omega / \mathrm{ft}$. These resistances do not change by more than $10 \%$ when cooled to $4.2 \mathrm{~K}$ due to the excellent thermal coefficient of manganin [84]. Any of the resistors could be removed from the filter circuit by breaking a lead bridge in series with each resistor. Three 
strings (not shown), running to the top of the probe, were connected to the bridges, allowing one to break any of the bridges while leaving the probe on the cryogenic bath. A twisted pair of 32 AWG insulated copper wire was used to connect the shunt resistor board to the coupling coil of the SQUID. These wires, like the pickup coil leads, were also shielded by a $1 \mathrm{~mm}$ o.d. $60 / 40$ lead-tin tube. The copper conductor on the fiberglass board did not exhibit any peeling despite many temperature cycles and no problems were observed with the entire shunt resistor assembly.

The samples were firmly packed into $5.0 \mathrm{~mm}$ o.d. thin-walled glass NMR tubes. The inner diameter of these NMR tubes are $4.27 \mathrm{~mm}$. The sample lengths are usually 0.5 ", and usually 2 or more are packed into the same NMR tube with 0.25 " spacers in between. These NMR tubes are then afixed to the end of a sample stick as shown in Figure 4.4. Usually, we applied a judicious amount of Apiezon vacuum grease to the Teflon and Nylon sections of the sample stick and cut slots in the NMR tubes at these corresponding locations. Doing so helped keep the sample tube attached to the stick when pulling the sample stick out of a cold probe.

\subsection{Cryostat and Room Temperature Shielding}

The stainless steel cryostat (dewar) used in this work was originally designed for high-field NMR, as previously mentioned. The capacity of the dewar was about 10 liters of liquid helium, which would allow 5 hours of operation. The liquid helium level was measured with a standard liquid helium probe and meter (American Magnetics 110) during the helium fill and periodically during operation. The helium bath was insulated with a liquid nitrogen jacket. A $\mu$-metal shield was wrapped outside the dewar to reduce the ambient magnetic field, at the sample region, by 


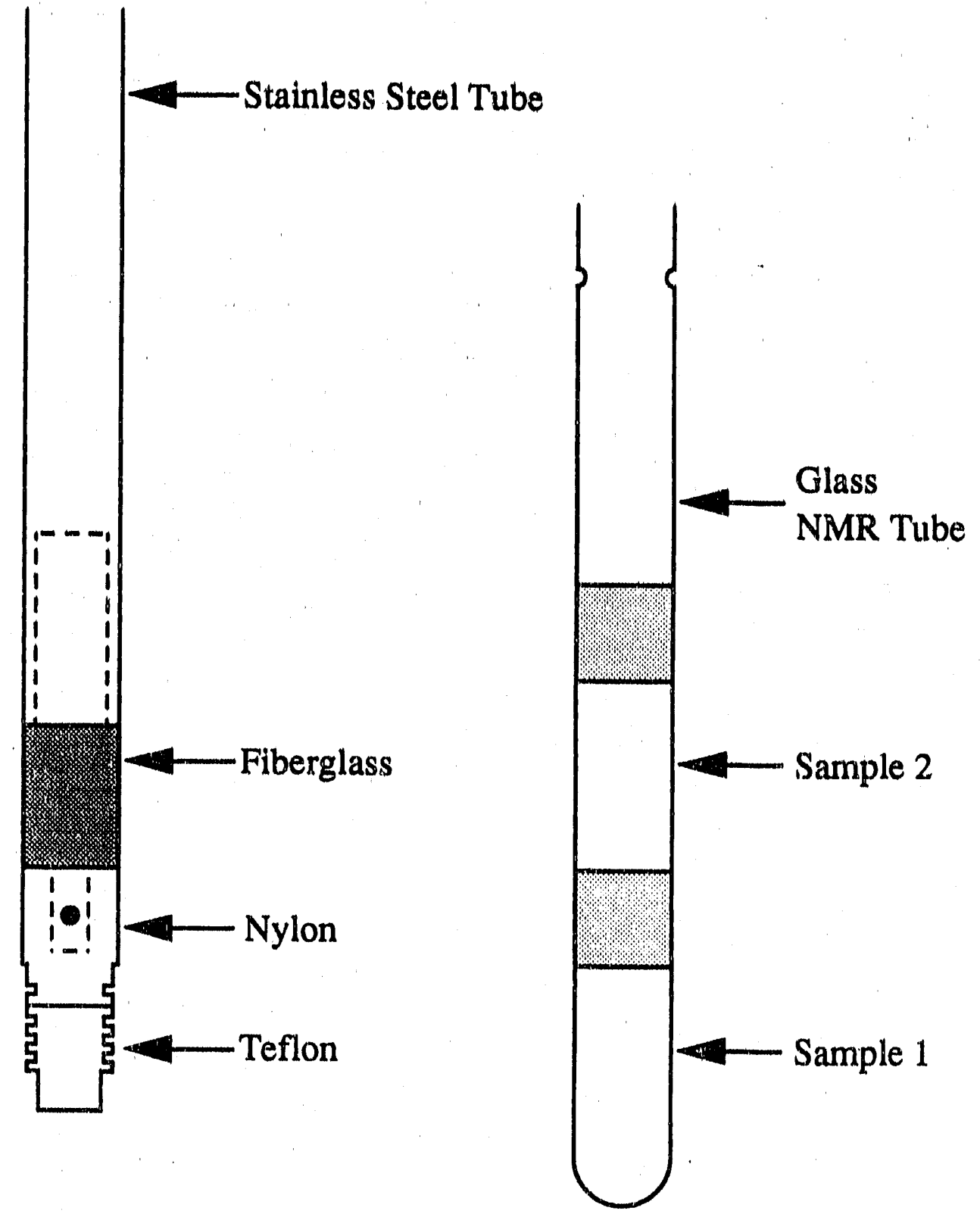

Figure 4.4: Sample tube and stick. The stick (left) is inserted into the glass sample tube. A thin film of vacuum grease keeps the stick and tube attached at cold temperatures. 
a factor of about 25 . This shield prevented the trapping of small magnetic fields during the liquid helium fill.

Experiments in this work were performed in an environment containing many sources of noise, such as several computers and pulsed NMR spectrometers in the same room, and pulsed laser systems in neighboring rooms. The dewar, along with the SQUID and probe, and feedback unit were placed inside the oage during operation. The Faraday cage helped filter out any high-frequency interference. The cage was constructed of copper mesh stapled to a wood frame with approximate dimensions of 8 feet in height and 6 feet in width and depth. No devices requiring the use of $110 \mathrm{~V}$ ac power were used inside the cage during operation of the spectrometer. Connections between the inside of the cage and the outside passed through a thick copper plate, either through feedthrough BNCs or 13-pin locking Bendix connectors (for the SQUID electronics).

\section{5 rf Sources and Data Acquisition}

Several sources of radiofrequency radiation were used in this work. The earliest sources were previously described in detail [85] and is omitted from this discussion. The most recent, and best, source of rf was a Hewlett-Packard 3326A Two-Channel Synthesizer. It is capable of supplying up to $10 \mathrm{~V}$ of $\mathrm{rf}$ into a $50 \Omega$ impedance load at frequencies from dc to $13 \mathrm{MHz}$. Sweep times were adjustable from $1 \mathrm{~ms}$ to 1000 $\mathrm{s}$ for any sweep width within the $13 \mathrm{MHz}$ bandwidth. An especially convenient operating mode was the two-phase mode where the two channels sweep together with an adjustable phase difference of $0^{\circ}$ to $720^{\circ}$. This permitted the generation of left or right circularly polarized rf. The frequency synthesis is produced digitally, 
making it easy to set all sweep parameters to any specifled value and making them reproducible. The rf synthesizer was also controllable by the computer using a HPIB interface.

The signal from the sample comes from the SQUID probe (BTi Model DP probe with Model DSQ Hybrid SQUID) and passes through the feedback unit (BTI Model 400) [82]. The operational features of the feedback unit were as described in Chapter 3. Then the signal goes to the SQUID control unit (BTI Model 40) where it is amplified, by factors of $1,10,100$, or 1000 , and filtered, using bandwidths of $5 \mathrm{kHz}$ or $50 \mathrm{kHz}$. The bandwidth was usually set to $5 \mathrm{kHz}$. Since this bandwidth is still much larger than needed, the output voltage of the SQUID system is then sent through a home-built low-pass filter. Figure 4.5 is a circuit diagram of this room-temperature filter. The input impedance is $20 \mathrm{k} \Omega$ and is a floating input as recommended by BTi. The bandwidth of the filter can be set from $200 \mathrm{~Hz}$ to 0.02 $\mathrm{Hz}$ by powers of ten, and the gain is continuously variable from 10 to 120 .

Usually, a computer and data acquisition unit were used to collect the output of the low-pass fliter. This allowed for more accurate signal collection, signal averaging, and the necessary signal processing (such as subtracting reverse sweeps from forward sweeps). An AT-clone personal computer (Leading Edge Model MH) controlled the Rapid Systems data acquisition unit [86]. The signal was digitized by an 8bit digitizer (Rapid Systems R1000) and stored in RAM on the Rapid Systems interface card (Model R320). When the frequency sweep and data acquisition was complete, the data points were sent to the computer RAM and displayed on the screen. Frequency sweeps and further data manipulation were performed either by the extensively modified program from Rapid Systems or a separate custom 

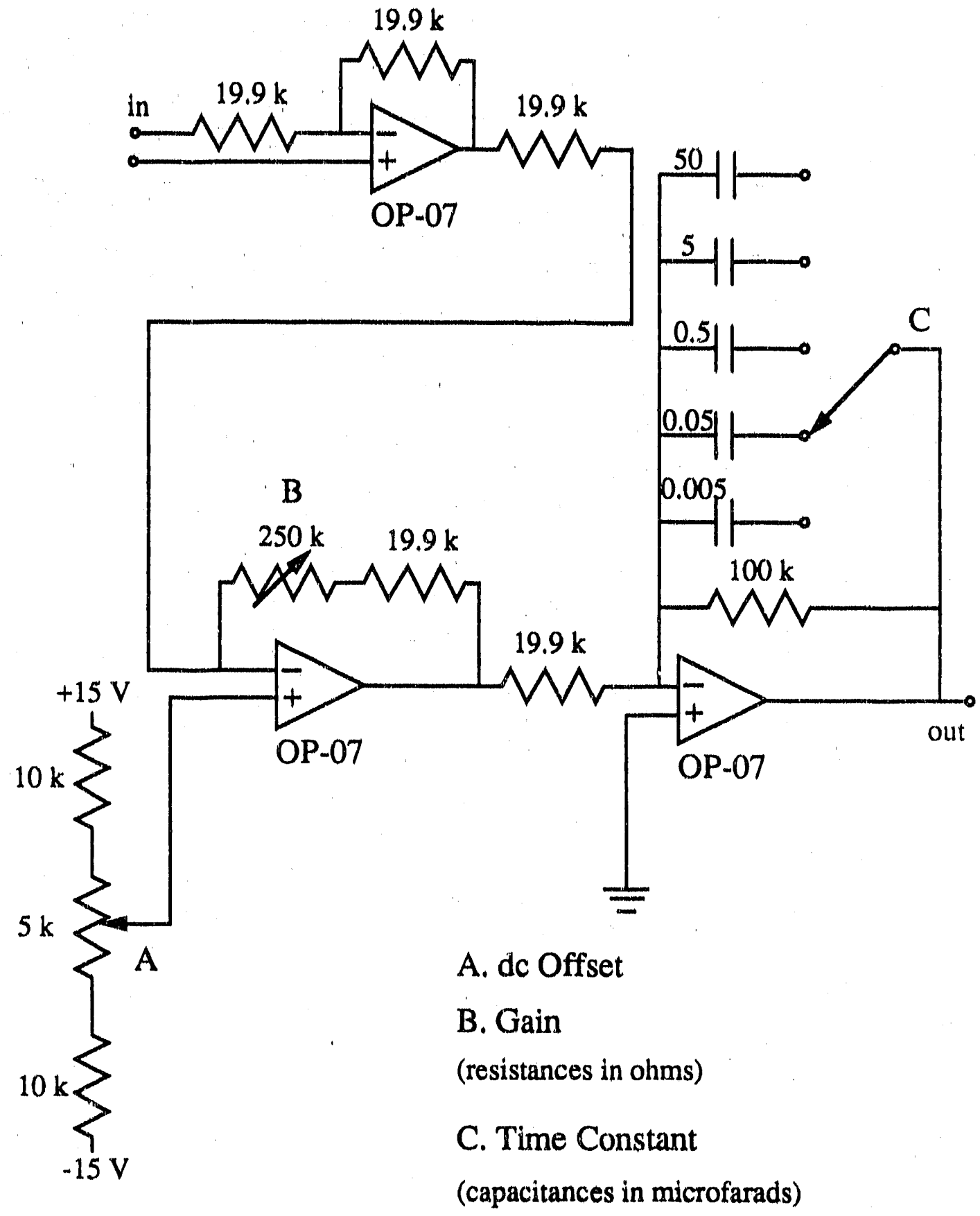

Figure 4.5: Schematic diagram of the low-pass filter through which the dc SQUID output voltage is passed. The filter provides additional amplification and filtering. 
program, both written in Turbo Pascal. The latter program allowed the user to plot the data or the screen, correct the spectrum of linear baseline drift, add and subtract spectra, etc. The Rapid Systems unit was also capable of performing very fast Fast Fourier Transforms using a TMS 32010 processor on the interface board. This was sometimes utilized for diagnostic purposes on the SQUID system. In a few cases, a chart recorder (Fisher Scientific 5000) was used to record the output of the low-pass filter. In this case, one had to be aware that the recorder had an upper frequency response of $4 \mathrm{~Hz}$ only.

\subsection{Spectrometer Performance}

It is of interest to determine the noise spectrum of the spectrometer, which may give one an idea of the source of the noise. To do this, we take the Fourier transform of the output of the SQUID control unit. The rf radiation is off at this point and the output does not pass through the low-pass filter. The noise is white down to about $1 \mathrm{~Hz}$ as shown in Figure 4.6. However, the noise amplitude increases sharply below this frequency [47]. If we assume a resonance linewidth of $20 \mathrm{kHz}$ and a sweep rate $\therefore: 1 \mathrm{kHz} / \mathrm{s}$, it can be said thrt our signal has an oscillation frequency of $0.05 \mathrm{~Hz}$. This suggests that further lock-in detection and signal modulation schemes may help improve the signal-to-noise ratio.

It is also of importance to know how much coupling exits between the rf and pickup coils. The best that can be theoretically achieved is zero coupling at all rf frequencies, however, this is hard to attain in practice. Extensive measurements of this coupling were made, for various shunt resistances, and the results are shown in Figure 4.7. The attenuation factors are those provided by the shunt resistor when a 

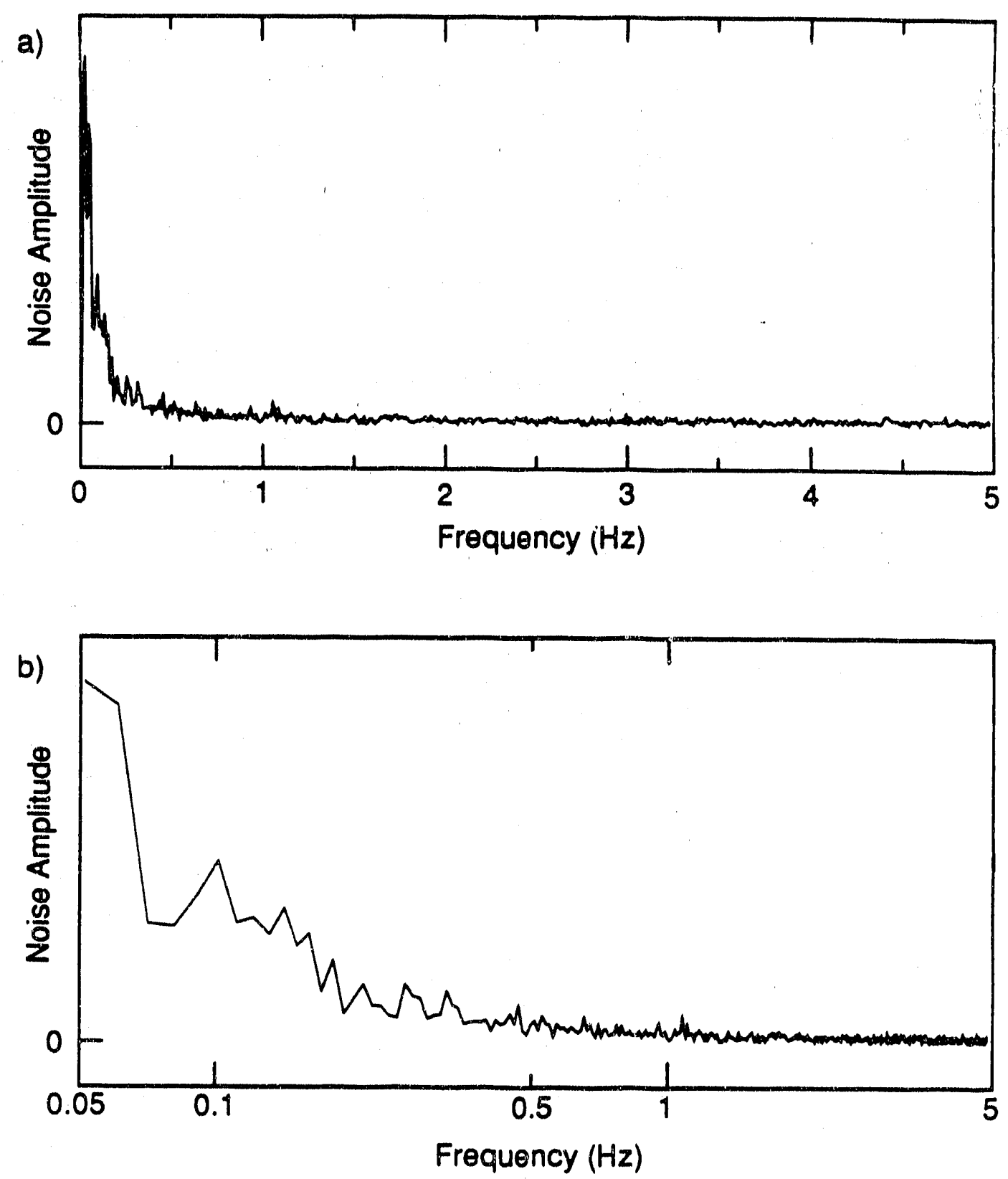

XOL $8911-4299$

Figure 4.6: a) dc SQUID output voltage noise amplitude versus frequency; b) Noise amplitude versus logarithm of frequency, showing an approximate $\frac{1}{f}$ dependence. 


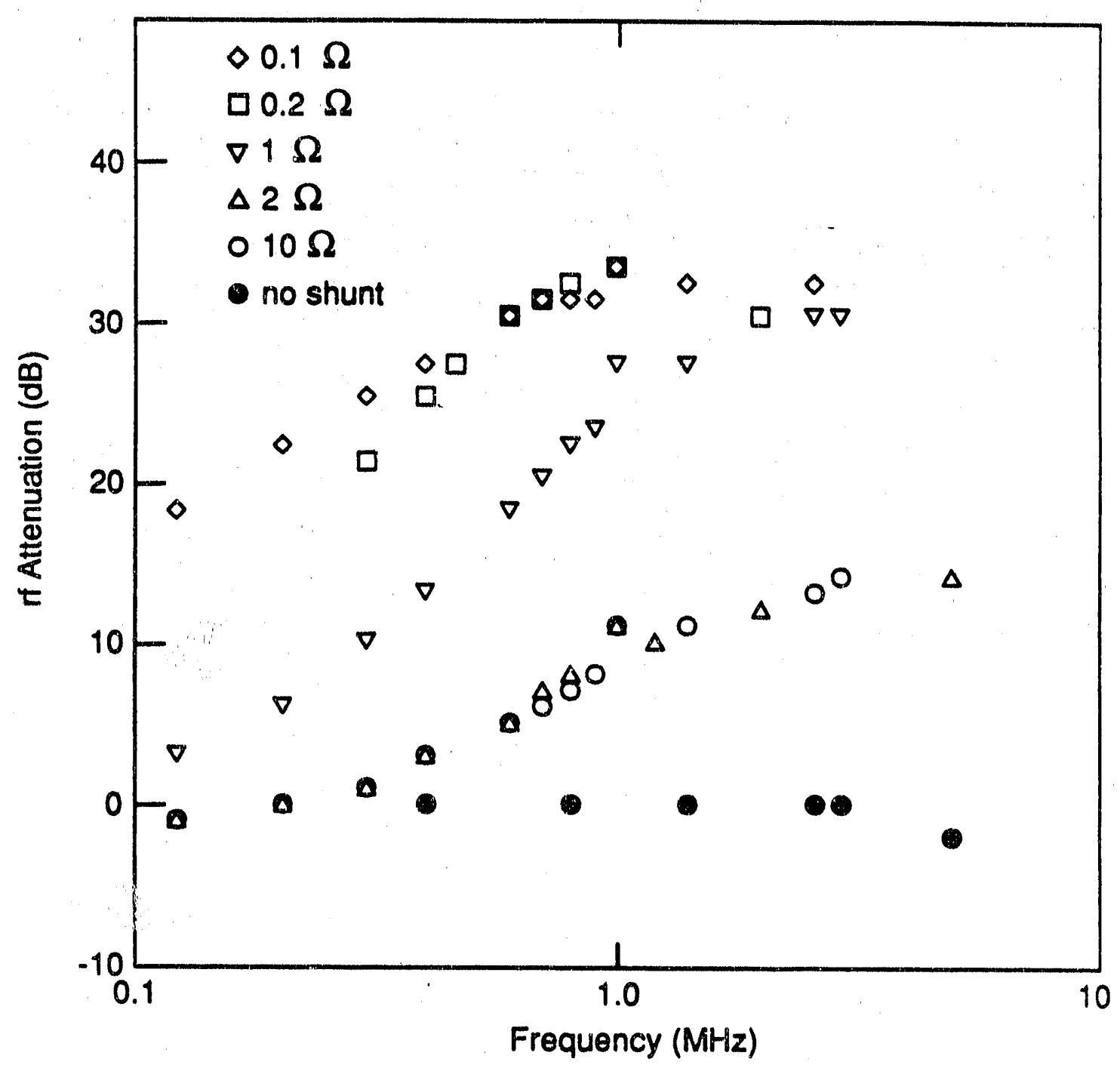

XBL $8911-4298$

Figure 4.7: Amount of $r f$ attenuation provided by the shunt resistor at various frequencies and for various shunt resistances. This gives a measure of the of coupling to the dc SQUID. This figure shows that the shunt resistor attenuates high-frequency interference and better attenuation is achieved with lower resistances. However, lower resistances produce larger amounts of flux noise. 
fixed rf voltage is sent through the rf coils, while keeping the flux-voltage curve at a fixed, predetermined amplitude. It is necessary to assume that all the if frequencies used are equally effective in reducing the amplitude of the flux-voltage curve. This figure shows that the shunt resistor does act as a low-pass filter and that reducing the frequency of the $\mathrm{rf}$ increases the noise, since the shunt resistor does not filter effectively at low frcquencies. As expected, stronger rf fields generally result in higher noise levels due to averaging of the flux-voltage curve. Using Figure 4.7, Equation 4.3 , and assuming $L_{P}=1.3 \mu \mathrm{H}$, the calculated $3 \mathrm{~dB}$ point of the filter is $200 \mathrm{kHz} / \Omega$.

A comparison of the noise expected and the actual noise observed can also be made. The measured noise in the output voltage of the SQUID, under typical operating conditions (10 G dc magnetic field, $10 \mathrm{mG}$ rf field, $2 \mathrm{~Hz}$ filter bandwidth, and $10^{4}$ overall gain), is approximately $50 \mathrm{mV} / \sqrt{\mathrm{Hz}}$ in the white noise region. If we take the stated intrinsic noise of the SQUID system, $1.2 \times 10^{-5} \Phi_{0} / \sqrt{\mathrm{Hz}}$, and the flux to voltage gain for the SQUID under the above conditions, $51 \mathrm{~V} / \Phi_{0}$, we calculate $0.61 \mathrm{mV} / \sqrt{\mathrm{Hz}}$ for the expected noise. Several modifications to the spectrometer did not substantially lessened this discrepancy.

Another way of gauging the spectrometer's performance is to compare the expected signal amplitudes to the experiment nl signal amplitudes. It was necessary to first empirically determine the overall gain of the spectrometer. This can be done by measuring the change in the output voltage for a given change in the amount of flux through the pickup coil. A given amount of magnetic flux can be generated in the pickup coil by placing a solenoid, very closely approximating the dimensions of a typical sample, in the typical sample position and passing the determined amount 
of current through it. We observed a gain of $23 \mathrm{~V} / \mu \mathrm{G}$ for the spectrometer, where the gain of the SQUID control unit was 1000 and the gain of the low-pass filter was 10. This is the calibration factor which allows us to determine the absolute magnetization of samples. Table 4.1 shows the measured and expected magnetizations for two samples, and the agreement is reasonable. The expected values were calculated using Table 2.1 , and the assumption was made that the rf completely saturates the spin system and that all the spins in the sample contribute to the signal. Relatively fast cross-relaxation between the $+m$ and $-m$ manifolds may be responsible for the reduced signal intensity of the boron nitride sample.

If one wishes to use a SQUID system in a pulsed field experiment, it is necessary to determine the dead-time of the SQUID after the strong magnetic field pulse. The dead-time of the detector determines which resonances will be observeable, depending on the linewidth of the resonance. With a dead-time of $T_{d t}$, one can (nominally) observe resonances with linewidths of $\frac{1}{T_{d t}}$ or less [87]. To determine the dead-time of our SQUID, we placed a Helmholtz pair of rf coils around a gradiometer pickup coil. A Cober Model 606P pulse generator, with a $200 \Omega$ resistor in series with the rf coil, was used to supply the dc magnetic field pulse with a field to voltage ratio of $0.05 \mathrm{G} / \mathrm{V}$. During the pulse, the SQUID was electronically decoupled from its preamplifier using a blanking line to the SQUID control unit. Figure 4.8 shows dead-times of about $50 \mu$ s for a $50 \mathrm{~V}$ pulse and $100 \mu \mathrm{s}$ for a $2000 \mathrm{~V}$ pulse. This tells us that with a $100 \mathrm{G}$ dc field pulse, one can observe resonances with linewidths of $10 \mathrm{kHz}$ or less. 


\begin{tabular}{|c|c|c|}
\hline & \multicolumn{2}{|c|}{ Z-Magnetization } \\
Sample & Calculated $(\mu \mathrm{G})$ & Measured $(\mu \mathrm{G})$ \\
\hline \hline $\begin{array}{c}\alpha \text {-Alumina crystal }\left({ }^{27} \mathrm{Al}\right) \\
\text { Boron nitride }\left({ }^{11} \mathrm{~B}\right)\end{array}$ & 0.23 & 0.19 \\
\hline
\end{tabular}

Table 4.1: Calculated and measured z rnagnetizations for two samples. 
a)

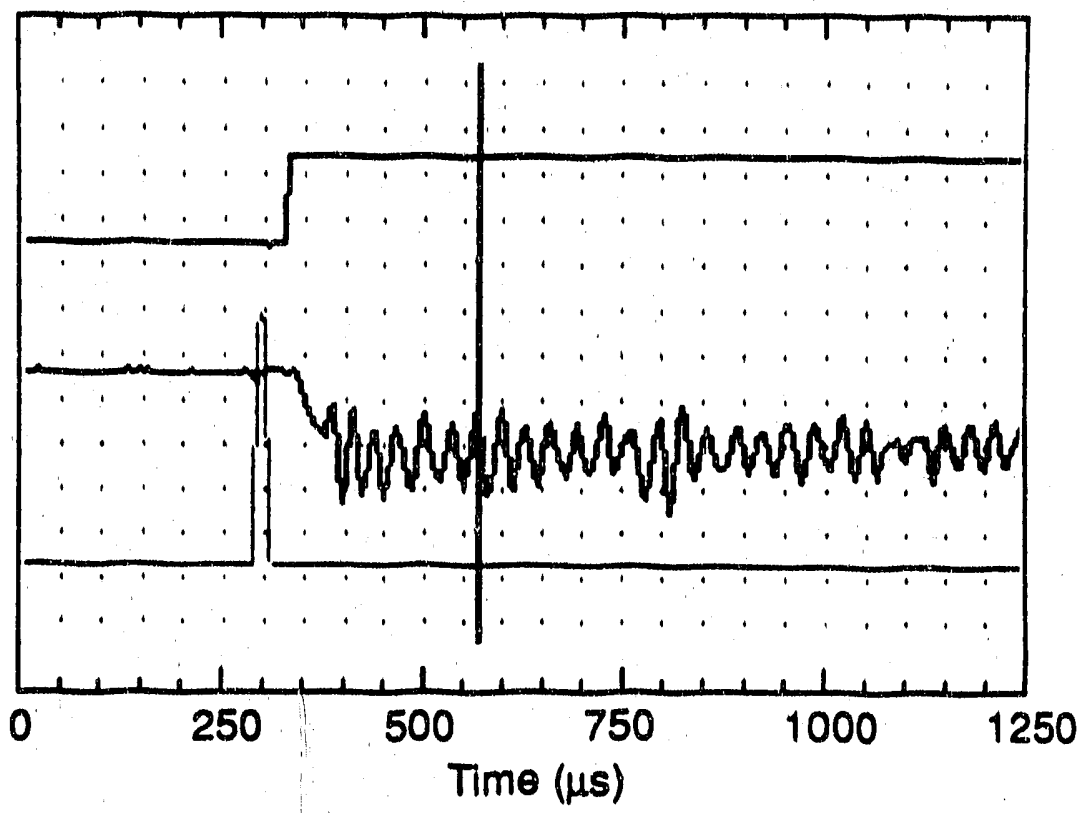

b)

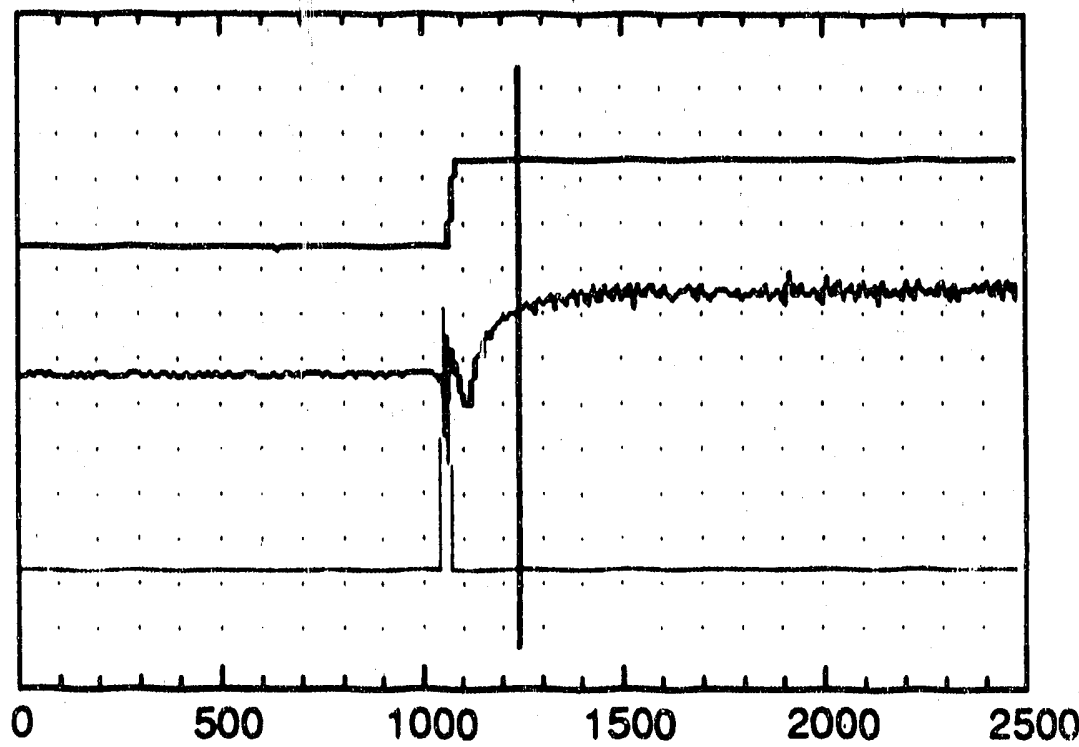

Time $(\mu s)$

XBL 8911-4387

Figure 4.8: Measurement of recovery times of the dc SQUID system after a short magnetic field pulse. The voltages of the pulses are a) $50 \mathrm{~V}$ and b) 2000 $\mathrm{V}$ (bottom traces), and the blanking line of the SQUID (top traces) is kept on during the pulse. The output voltage of the SQUID system (middle traces) show dead-times of approximately a) $50 \mu \mathrm{s}$ and b) $100 \mu \mathrm{s}$. 


\section{Chapter 5}

\section{$I=\frac{3}{2}$ NQR Results}

\subsection{Introduction}

Most of the boron work in this chapter concentrates on ${ }^{11} \mathrm{~B}\left(I=\frac{3}{2}\right)$ since ${ }^{10} \mathrm{~B}$ has spin $I=3$, which we will see, is less amenable to study with this SQUID NQR technique. In the $\mathrm{B}_{2} \mathrm{O}_{3} \cdot \mathrm{xH}_{2} \mathrm{O}$ section, resonances for the $\mathrm{BO}_{3}$ group are shown. The $\mathrm{BO}_{3}$ units have resonance frequencies around $1200 \mathrm{kHz}$ and their asymmetry parameters have been determined to be relatively small $[28,88,89]$. It is shown that the resonance frequency is a sensitive probe of the environment of the $\mathrm{BO}_{3}$ units. There is evidence of $\mathrm{BO}_{4}$ groups in some of these samples, however, their resonances frequencies are too low to be detected by SQUID NQR since the electric quadrupole moment of ${ }^{11} \mathrm{~B}$ is relatively small and distortions from tetrahedral symmetry are usually not large. Thus, this discussion is restricted to the $\mathrm{BO}_{3}$ units. Table 5.1 shows some relevant data on the physical properties of boron and aluminum. 


\begin{tabular}{|c|c|c|c|}
\hline Physical Property & \multicolumn{3}{|c|}{ Nucleus } \\
Spin $(I)$ & ${ }^{10} \mathrm{~B}$ & ${ }^{11} \mathrm{~B}$ & ${ }^{27} \mathrm{Al}$ \\
\hline \hline Natural abundance (\%) & 3 & $\frac{3}{2}$ & $\frac{5}{2}$ \\
Gyromagnetic ratio, $\gamma(\mathrm{Hz} / \mathrm{G})$ & 19.6 & 80.4 & 100.0 \\
Electric quadrupole moment, $e Q\left(\times 10^{-24} \mathrm{~cm}^{2}\right)$ & $7.4 \times 10^{-2}$ & $3.55 \times 10^{-2}$ & 0.149 \\
\hline \hline
\end{tabular}

Table 5.1: Some relevant physical data on ${ }^{10} \mathrm{~B},{ }^{11} \mathrm{~B}$, and ${ }^{27} \mathrm{Al}$. The quadrupole moments listed are the accepted values. 


\subsection{Boron Nitride, $B N$}

Boron Nitride (BN), being isoelectronic with carbon, also has two phases: hexagonal and cubic [88]. The cubic phase is structurally identical to diamond where all the units are tetrahedral. This makes the cubic phase very stable and hard, and also makes any quadrupolar interaction disappear due to the very high symmetry of the system [9]. The system we studied, the hexagonal phase, consists of boron bonded to three nitrogen nuclei to form planar trigonal groups and vice versa for the nitrogen nuclei. These planar groups are linked together to form sheets of six-membered rings analagous to graphite. The only structural difference between graphite and the boron nitride hexagonal phase is that in the boron nitride the nuclei are all stacked on top of each other $[90,91]$. This is unlike graphite where the sheets are layered in a staggered fashion.

The bonding in the BN hexagonal phase has been discussed by Silver and Bray [92] and it should be immediately evident that the quadrupolar interaction is very highly axially symmetric due to the $\mathrm{C}_{3 V}$ axis through each boron nucleus. The z-axis of the quadrupolar interaction is coincident with this $\mathrm{C}_{3 V}$ axis [92]. Three different samples of BN were studied, one from Alpha products [93] and two from ESPI [94]. The two powder samples (Alpha and ESPI) were very fine white powder of high purity ( $>99 \%$ ). The third sample (ESPI) was a hot-pressed rod of unspecified purity.

The powder sample from Alpha products gave a very clean ${ }^{11} \mathrm{~B}$ peak at 1466 $\pm 2 \mathrm{kHz}$ as shown in Figure 5.1, in good agreement with room temperature measurements of $1450 \pm 50 \mathrm{kIr}$ [92]. No other bumps or smaller peaks were seen in the spectrum of this sample. The spectrum of the BN powder sample from ESPI 


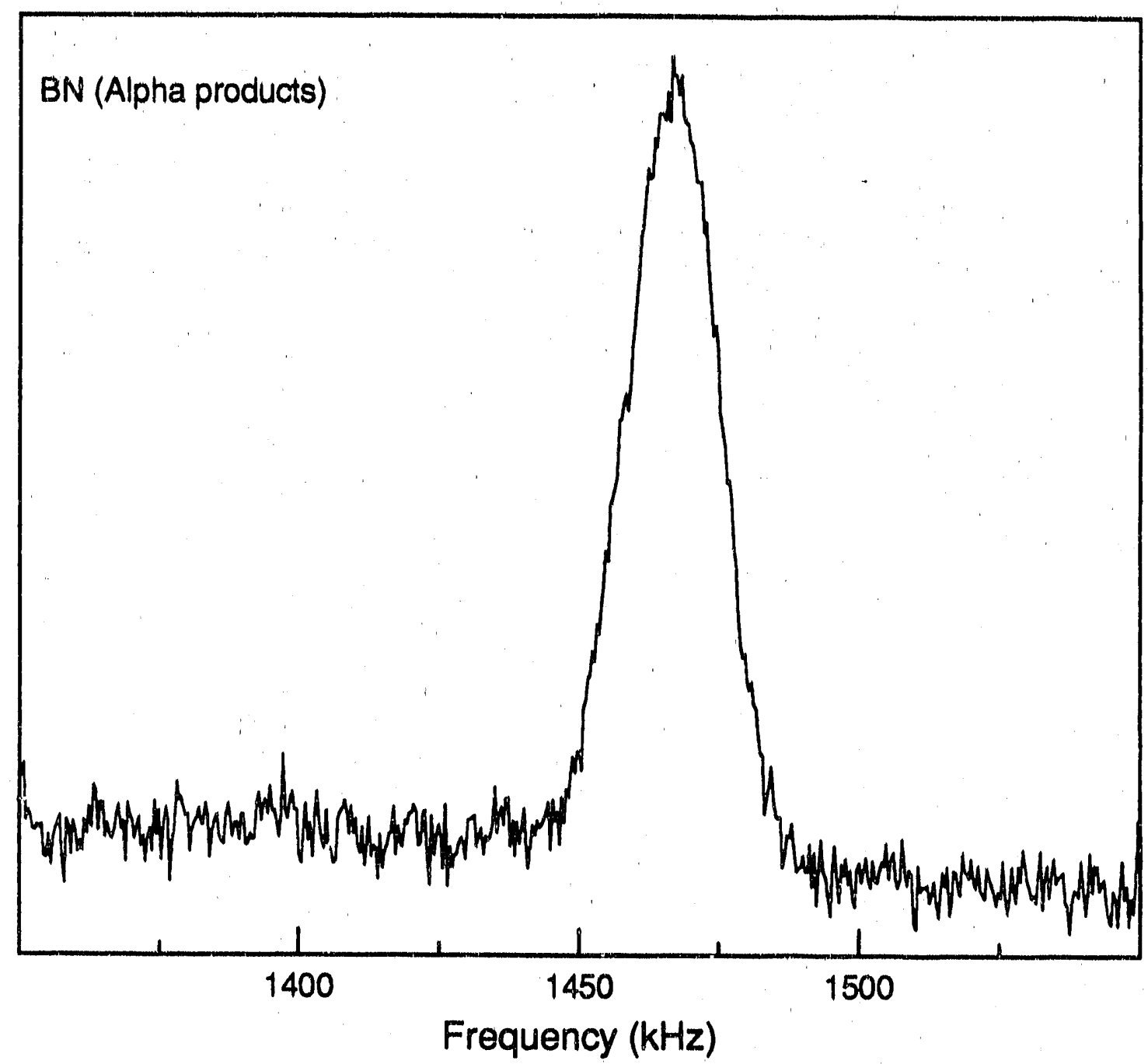

Figure 5.1: Spectrum of boron nitride (BN) powder from Alpha products. The ${ }^{11} \mathrm{~B} N Q R$ resonance frequency is $1466 \pm 2 \mathrm{kHz}$. The dc magnetic field was $1.5 \mathrm{G}$ and a total of 40 forward and reverse sweeps were used to obtain this spectrum. 
is shown in Figure 5.2 and it also yielded a main peak at $1466 \pm 2 \mathrm{kHz}$. However, this spectrum has two other regions of interest: near $1300 \mathrm{kHz}$ and a low frequency shoulder on the main peak. The hump near $1300 \mathrm{kHz}$ has been assigned to small amounts of impurities of the $\mathrm{B}_{2} \mathrm{O}_{3} \cdot \mathrm{xH}_{2} \mathrm{O}$ variety since $\mathrm{BN}$ hydrolyzes when exposed to water [95] and this is where the $\mathrm{B}_{2} \mathrm{O}_{3} \cdot \mathrm{xH}_{2} \mathrm{O}$ resonances appear as shown in the following section.

In order to obtain a better spectrum of the main peak and its low frequency shoulder, we reduced the dc magnetic field to $1.5 \mathrm{G}$ and the spectrum of the ESPI hot-pressed rod of BN is shown in Figure 5.3. This hump near $1300 \mathrm{kHz}$ does not appear in the spectrum of the hot-pressed rod sample, most likely because the BN units are not exposed to moisture while in the rod form. The small shoulder on the main peak is now better resolved and it has not previously been reported. By fitting this spectrum to the sum of two Gaussians, we found that the shoulder accounted for approximately $30 \%$ of the overall intensity. It was hypothesized that the shoulder originated from defects in the plane-layering scheme at regular intervals. This was later ruled out on the basis of two arguments. Previous work had indicated that these layer shifts are randomly distributed $[90,91]$. Secondly, X-ray diffraction of the ESPI powder sample showed it to be as crystalline as the Alpha sample which showed no shoulder [96]. A clean BN spectrum from the Alpha sample also ruled out the possibility that the shoulder was a ${ }^{14} \mathrm{~N}$ resonance which could appear in this frequency region. Also, the shoulder is not a ${ }^{10} \mathrm{~B}$ resonance since it would appear on the high frequency side of the main peak, due to the very low asymmetry parameter of BN [92]. At this point, we are still unable to identify the source of the shoulder in the ESPI samples. 


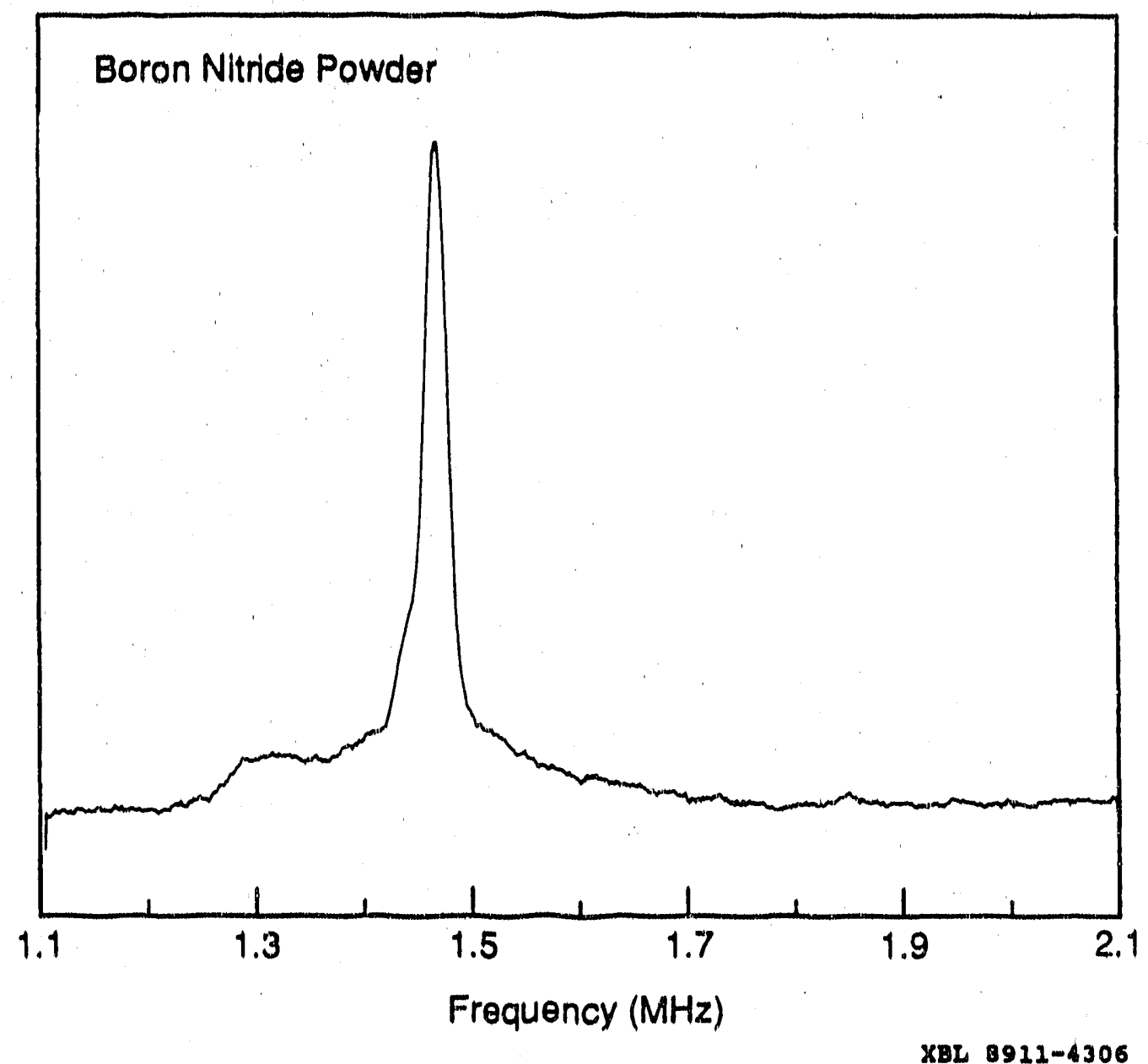

Figure 5.2: ${ }^{11} \mathrm{~B}$ spectrum of boron nitride (BN) powder from ESPI. A total of 20 sweeps were used and the do field was about $4 \mathrm{G}$. The broad hump near $1300 \mathrm{kHz}$ is from ${ }^{11} \mathrm{~B}$ in $\mathrm{B}_{2} \mathrm{O}_{3} \cdot \mathrm{xH}_{2} \mathrm{O}$, hydrolyzed $\mathrm{BN}$. 


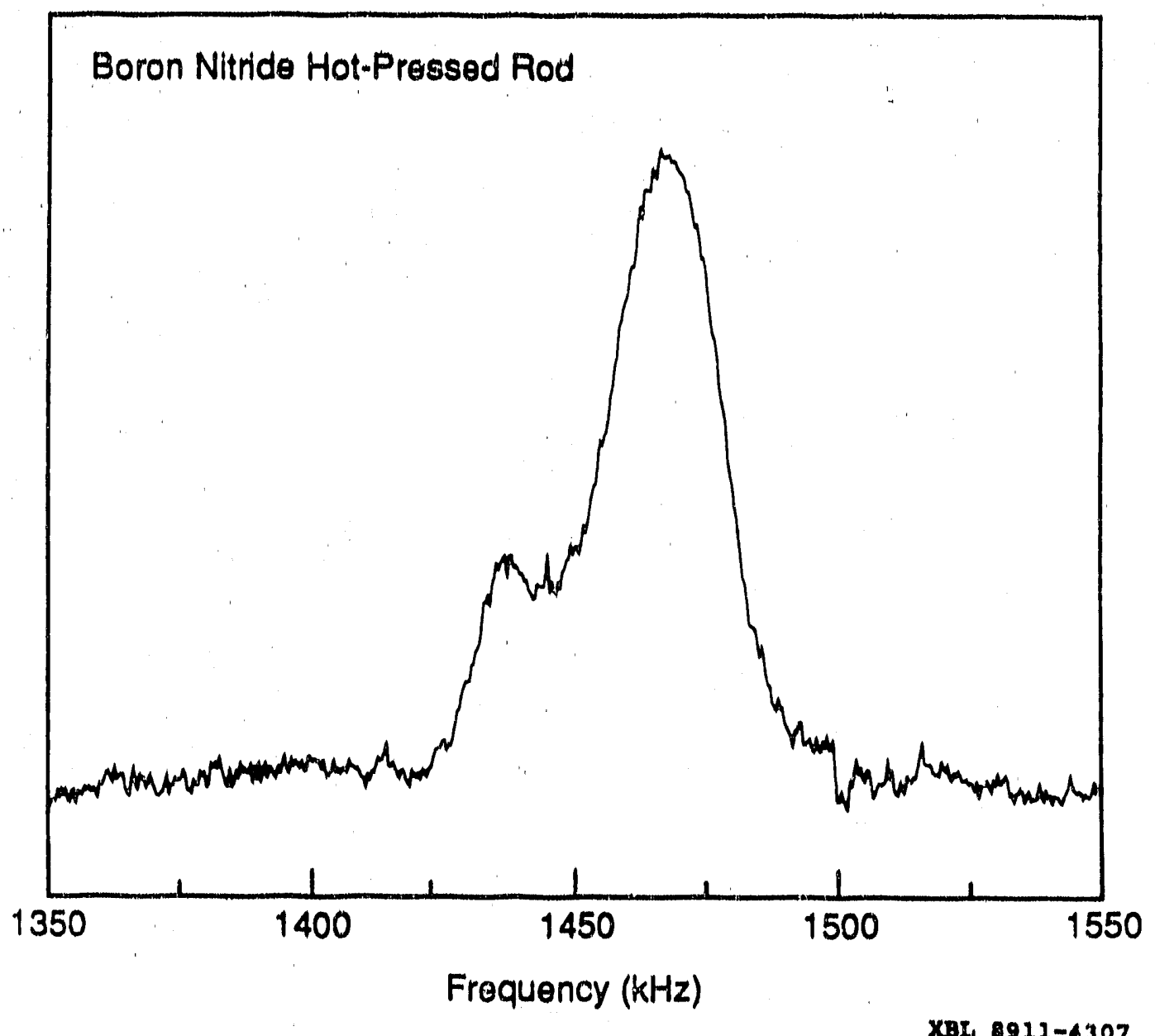

Figure 5.3: High resolution ${ }^{11} \mathrm{~B}$ spectrum of a hot-pressed rod of BN (ESPI). The dc magnetic field was $1.5 \mathrm{G}$ and 32 sweeps were used to obtain this spectrum. The source of the low-frequency shoulder is still unknown (see text). 
In the process of obtaining the BN spectra, we came across a case where moderately strong dipolar coupling forced us to acquire the spectra using a relatively fast sweep rate. The boron nuclei in $\mathrm{BN}$ are in fairly close proximity to each other and allows for moderately strong dipolar coupling. 'This increase in dipolar coupling allowed cross-relaxation between manifolds to occur at a high rate. In order to reduce the effects of this, we had to sweep the rf frequency at a relatively high rate of $4 \mathrm{kHz} / \mathrm{sec}$, while $1 \mathrm{kHz} / \mathrm{sec}$ was usually adequate with other samples.

\subsection{Boron Hydrates, $\mathrm{B}_{2} \mathrm{O}_{3} \cdot \mathrm{xH}_{2} \mathrm{O}(\mathrm{x}=3,1$, and 0$)$}

In this section, we present some new information on the $\mathrm{B}_{2} \mathrm{O}_{3} \cdot \mathrm{xH}_{2} \mathrm{O}$ system, where $x=3,1$, and $0[27,89,92,97,98,99,100,101,102]$, which we were able to get after obtaining their boron NQR spectra.

\subsubsection{Boric acid}

Boric acid $\left(\mathrm{B}_{2} \mathrm{O}_{3} \cdot 3 \mathrm{H}_{2} \mathrm{O}, \mathrm{H}_{3} \mathrm{BO}_{3}\right.$, or $\left.\mathrm{B}(\mathrm{OH})_{3}\right)$ has the boron nuclei surrounded by three oxygen atoms in the same plane, such that the O-B-O bond angle is very close to $120^{\circ}$ [103]. These planar $\mathrm{BO}_{3}$ units are linked together by hydrogen bonding between the oxygen atoms to form a flat layered network, Figure 5.4. X-ray diffraction and neutron scattering data show two distinct boron sities $\left[{ }^{+} 03,104\right]$ but they do not seem to be resolvable by NQR. The boric acid, obtained from J.T. Baker Chemical Co. [105], was dissolved in hot-distilled $\mathrm{H}_{2} \mathrm{O}$ then recrystallized to ensure complete hydration. The spectrum of boric acid (natural abundance ${ }^{11} \mathrm{~B}$ ) is shown in Figure 5.5. The main peak $\left({ }^{11} \mathrm{~B}\right)$ at $1288 \pm 2 \mathrm{kHz}$, is in excellent agreement with a previous value of $1288 \pm 4 \mathrm{kHz}$ [100]. The shoulder, from ${ }^{10} \mathrm{~B}$ nuclei, 


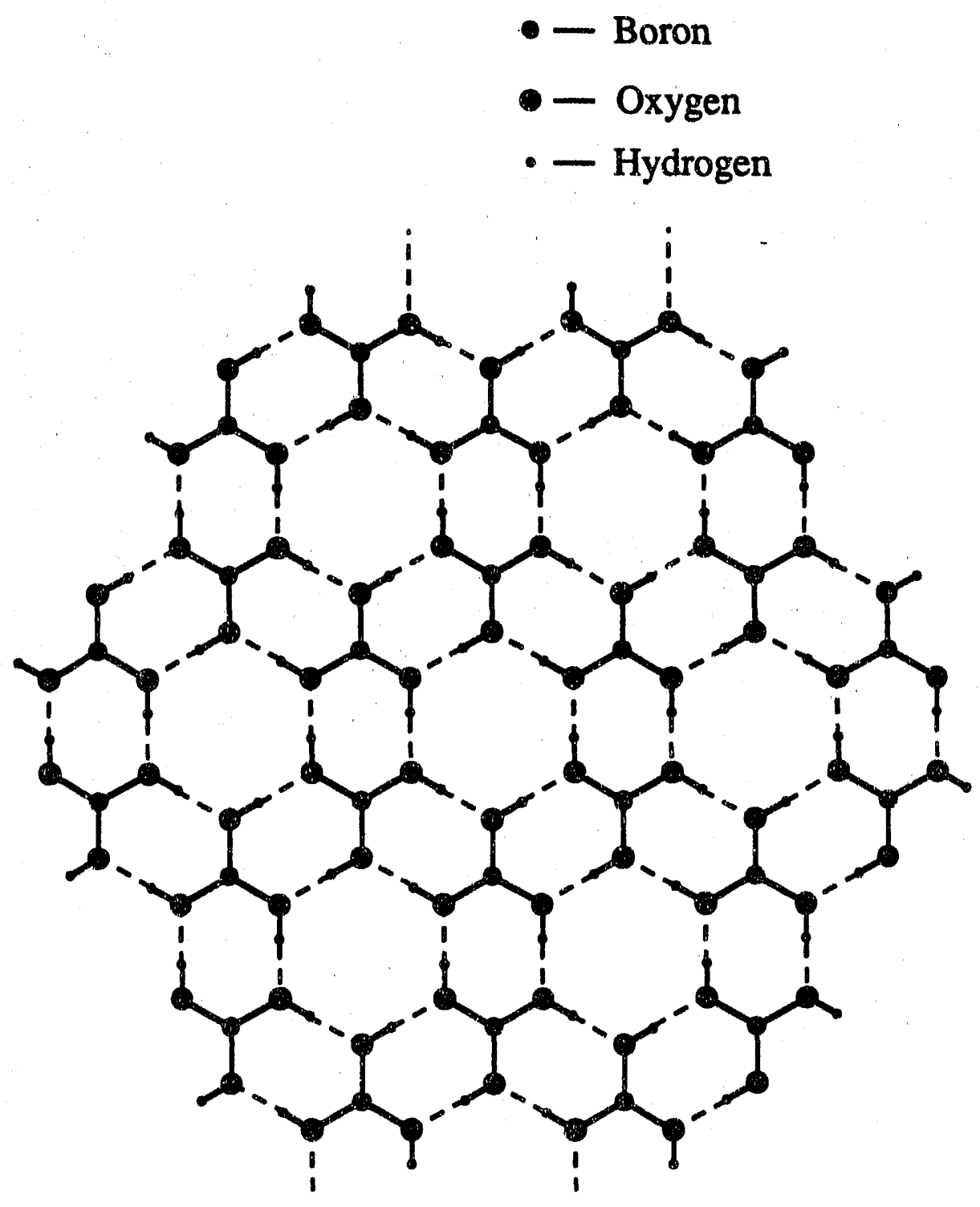

Figure 5.4: The flat sheets which make up boric acid $\left(\mathrm{B}_{2} \mathrm{O}_{3} \cdot 3 \mathrm{H}_{2} \mathrm{O}\right)$ are made of $\mathrm{H}_{3} \mathrm{BO}_{3}$ units joined by hydrogen bonding (dashed lines). The O-B-O angles are very close to $120^{\circ}$. 


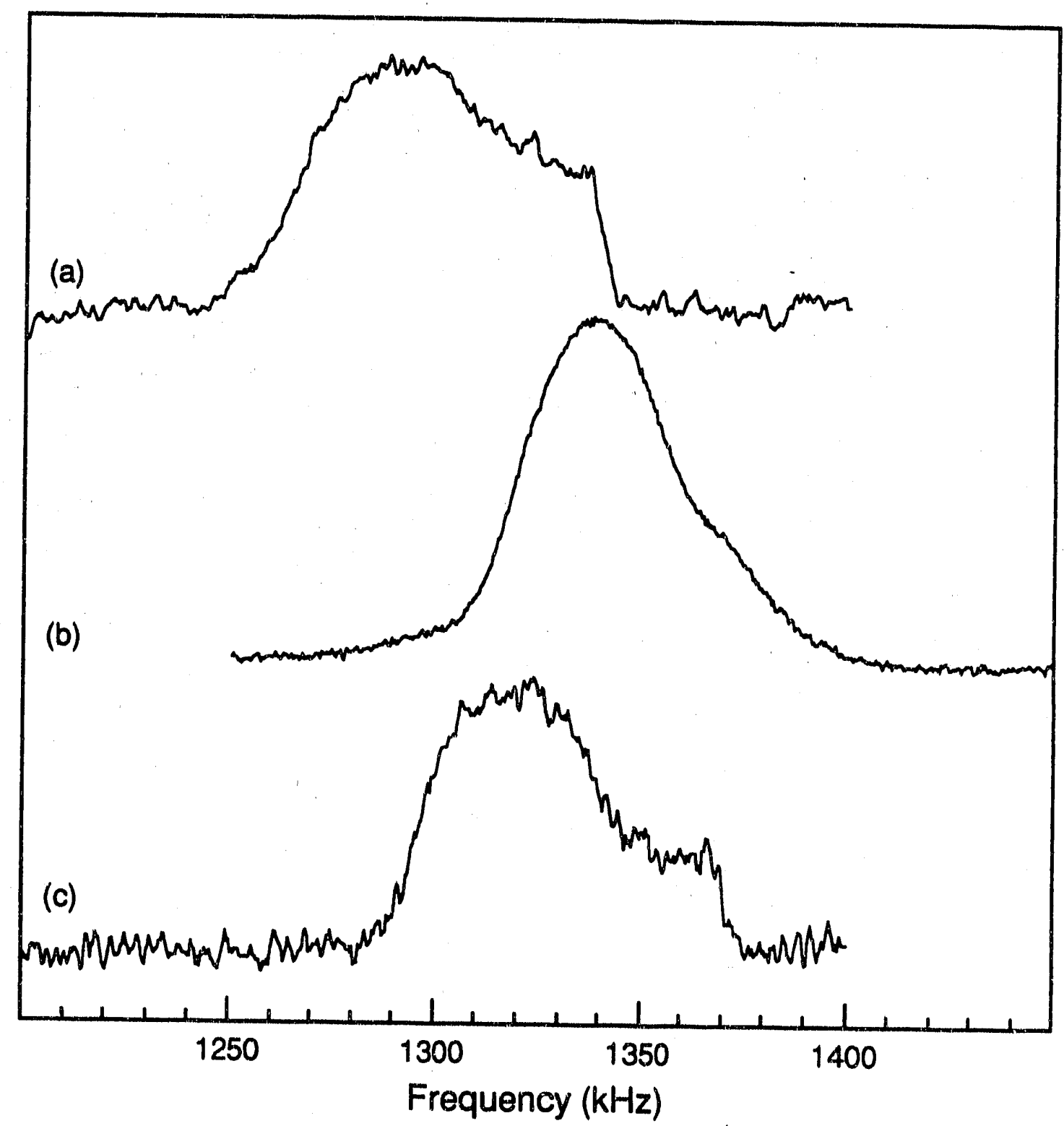

Figure 5.5: Boron NQR spectra of polycrystalline (a)boric acid $\left(\mathrm{B}_{2} \mathrm{O}_{3} \cdot 3 \mathrm{H}_{2} \mathrm{O}\right)$, (b) boron monohydrate $\left(\mathrm{B}_{2} \mathrm{O}_{3} \cdot \mathrm{H}_{2} \mathrm{O}\right)$, and (c)boron oxide $\left(\mathrm{B}_{2} \mathrm{O}_{3}\right)$. The spectra. are normalized to about the same height and the dc field was about $9 \mathrm{G}$. These samples are natural abundance in ${ }^{10} \mathrm{~B}$ and ${ }^{11} \mathrm{~B}$. The high-frequency shoulders, on all three spectra, are from the $\pm 2^{\prime} \leftrightarrow \pm 3^{\prime}$ transitions of ${ }^{10} \mathrm{~B}$. 
on the high frequency side of the main peak is discussed later.

\subsubsection{Boron monohydrate}

The orthorhombic form of $\mathrm{B}_{2} \mathrm{O}_{3} \cdot \mathrm{H}_{2} \mathrm{O}$ (III) was prepared by heating boric acid in an open container at $100^{\circ} \mathrm{C}$ for 24 hours $[89,95,106]$. The immediate environment of the boron nuclei is similar to that in boric acid except for a lower extent of hydrogen bonding $[88,95]$. The spectrum of $\mathrm{B}_{2} \mathrm{O}_{3} \cdot \mathrm{H}_{2} \mathrm{O}\left(\mathrm{HBO}_{2}\right)$ (natural abundance ${ }^{11} \mathrm{~B}$ ) is shown in Figure 5.5. Our measured ${ }^{11} \mathrm{~B}$ resonance frequency of $1336 \pm 2 \mathrm{kHz}$ is quite different from $1280 \mathrm{kHz}$, reported by Silver using simulations of NMR powder lineshapes [89]. This discrepancy may arise from the greater precision and accuracy of NQR in determining quadrupolar splittings or a difference in sample preparation. The small high frequency shoulder is again attributed to ${ }^{10} \mathrm{~B}$ and is discussed later.

\subsubsection{Boron oxide (crystalline)}

The polycrystalline sample of $\mathrm{B}_{2} \mathrm{O}_{3}$ was obtained from ESPI, then dried overnight at $70^{\circ} \mathrm{C}$ to ensure complete dehydration. $\mathrm{B}_{2} \mathrm{O}_{3}$ consists of $\mathrm{BO}_{3}$ groups joined by the oxygen atoms to form a three-dimensional network [88]. The measured ${ }^{11} \mathrm{~B}$ resonance frequency, Figure 5.5, of $1318 \pm 4 \mathrm{kHz}$ (natural abundance ${ }^{11} \mathrm{~B}$ ) appears to not have been previously measured. The high frequency shoulder in our spectrum is again assigned as a ${ }^{10} \mathrm{~B}$ transition.

Table 5.2 summarizes the measured ${ }^{11} \mathrm{~B}$ resonance frequency, resonance linewidth, and estimated $T_{1}$ of these three samples. No attempt was made to accurately determine the $T_{1}$ of these samples, so they should only be used as a guide. The long $T_{1}$ value for boric acid was observed in a variety of boric acid samples from different 


\begin{tabular}{|c|c|c|c|}
\hline \hline & \multicolumn{3}{|c|}{ Sample } \\
& $\mathrm{B}_{2} \mathrm{O}_{3} \cdot 3 \mathrm{H}_{2} \mathrm{O}$ & $\mathrm{B}_{2} \mathrm{O}_{3} \cdot \mathrm{H}_{2} \mathrm{O}$ & $\mathrm{B}_{2} \mathrm{O}_{3}$ \\
\hline \hline${ }^{11} \mathrm{~B}$ resonance frequency $(\mathrm{kHz})$ & 1288 & 1336 & 1318 \\
Linewidth $(\mathrm{kHz})$ & 50 & 40 & 40 \\
Estimated $T_{1}(\mathrm{~s})$ & 300 & 60 & 60 \\
\hline
\end{tabular}

Table 5.2: Measured resonance frequency, linewidth, and estimated $T_{1}$ for ${ }^{11} \mathrm{~B}$ in the three polycrystalline borates. 
sources. Attempts to reduce the $T_{1}$ of boric acid using paramagnetic doping were unsuccessful.

\subsubsection{Further boron hydrates}

In the previous sections, we observed high-frequency shoulders on the main peaks. It is the work of Butler and Brown which has allowed us to assign these shoulders to the high frequency $\left( \pm 2^{\prime} \leftrightarrow \pm 3^{\prime}\right)$ transitions of ${ }^{10} \mathrm{~B}$ [100]. This, along with the ${ }^{11} \mathrm{~B}$ resonances presented in the preceding sections, has allowed the determination of the quadrupolar parameters for the boron nuclei and gives additional information about their immediate environment.

The assignment of the shoulders to the ${ }^{10} \mathrm{~B}$ nuclei was based on two arguments. Butler and Brown observed, using a field-cycling technique, the $\pm 2^{\prime} \leftrightarrow \pm 3^{\prime}$ transitions of ${ }^{10} \mathrm{~B}$ in boric acid at $1335 \mathrm{kHz}$. This is in excellent agreement with our ${ }^{10} \mathrm{~B}$ resonance frequency of $1332 \mathrm{kHz}$. The second supporting piece of evidence to this assignment is the absence of the shoulders in the spectra of $99 \%{ }^{11} \mathrm{~B}$-enriched samples, obtained using our SQUID technique. Figure 5.6 show spectra of these ${ }^{11} \mathrm{~B}$-enriched samples. The possibility that the shoulder in $\mathrm{B}_{2} \mathrm{O}_{3} \cdot 3 \mathrm{H}_{2} \mathrm{O}$ is due to $\mathrm{B}_{2} \mathrm{O}_{3} \cdot \mathrm{H}_{2} \mathrm{O}$ was eliminated due to the small linewidth of the shoulder compared to the much larger linewidth of the $\mathrm{B}_{2} \mathrm{O}_{3} \cdot \mathrm{H}_{2} \mathrm{O}$ resonance.

A little theory on spin $I=3$ nuclei is in order at this point. As a reminder, the pure ${ }^{11} \mathrm{~B} N Q R$ resonance occurs at

$$
\omega_{Q, 1_{\mathrm{B}}}=\frac{e^{2} q Q}{2 \hbar}\left(1+\frac{\eta^{2}}{3}\right)^{\frac{1}{2}} .
$$

The energy levels of a spin $I=3$ nucleus, such as ${ }^{10} \mathrm{~B}$ (natural abundance $\approx 20 \%$ ), 


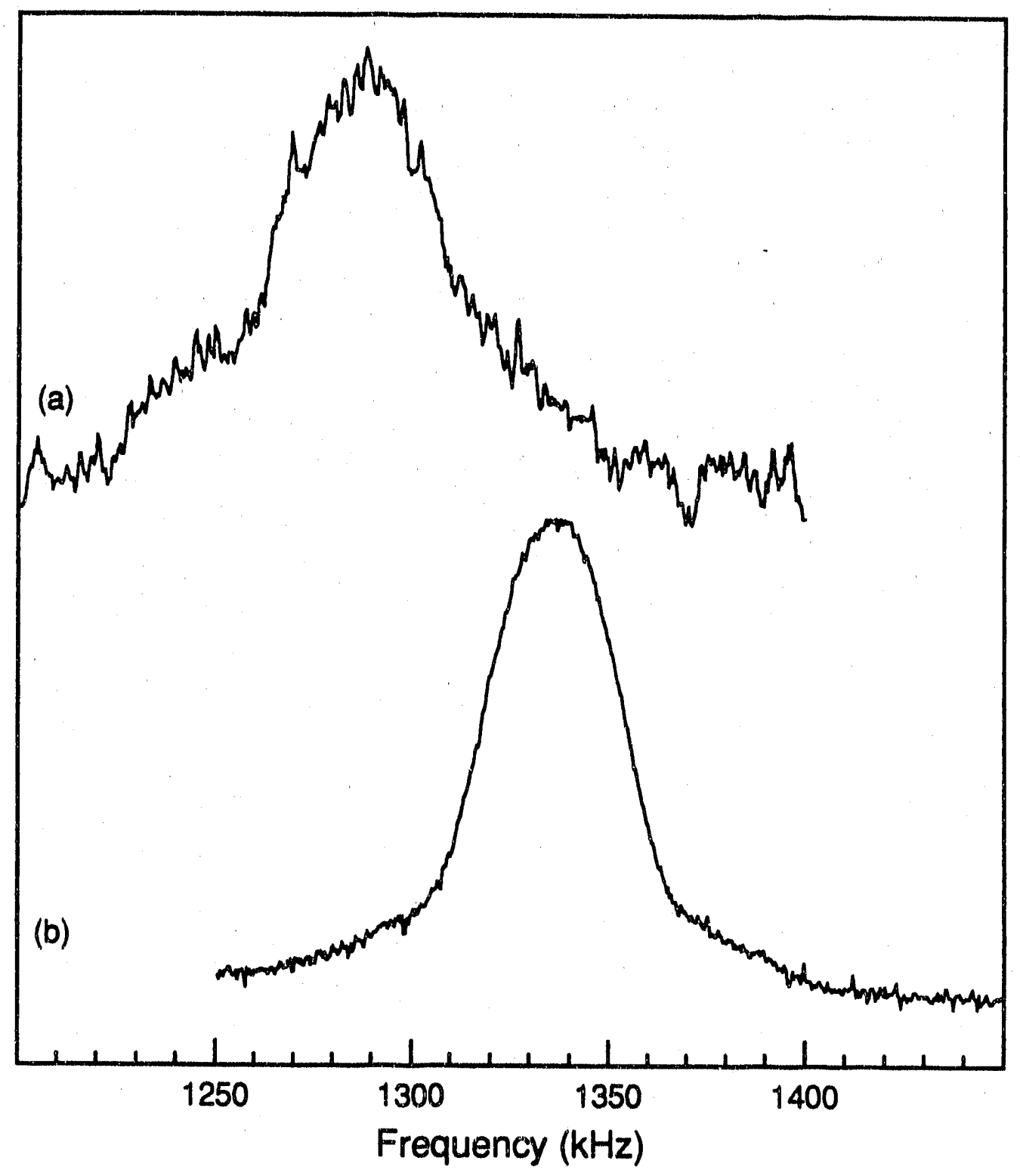

Figure 5.6: Spectra of $99 \%{ }^{11} \mathrm{~B}$-enriched (a) $\mathrm{B}_{2} \mathrm{O}_{3} \cdot 3 \mathrm{H}_{2} \mathrm{O}$ and $(\mathrm{b}) \mathrm{B}_{2} \mathrm{O}_{3} \cdot \mathrm{H}_{2} \mathrm{O}$ in a dc field of about $9 \mathrm{G}$. The high-frequency shoulders, due to the $\pm 2^{\prime} \leftrightarrow \pm 3^{\prime}$ transitions of ${ }^{10} \mathrm{~B}$, are absent. 
has been published by Creel [107] using the zero-order basis set

$$
\begin{aligned}
\left| \pm 3^{\prime}\right\rangle & =\frac{1}{\sqrt{2}}(|+3\rangle \pm|-3\rangle) \\
\left| \pm 2^{\prime}\right\rangle & =\frac{1}{\sqrt{2}}(|+2\rangle \pm|-2\rangle) \\
\left| \pm 1^{\prime}\right\rangle & =\frac{1}{\sqrt{2}}(|+1\rangle \pm|-1\rangle) \\
|0\rangle & =|0\rangle
\end{aligned}
$$

Only the $\pm 2^{\prime} \leftrightarrow \pm 3^{\prime}$ transitions are used in this work, and they appear at

$$
\begin{aligned}
\frac{\omega_{Q,+}}{A}=\frac{E\left(+3^{\prime}\right)-E\left(+2^{\prime}\right)}{A}= & 9+3 \eta+3\left[(4-\eta)^{2}+\frac{5 \eta^{2}}{3}\right]^{\frac{1}{2}} \\
& -6\left[1+\frac{5 \eta^{2}}{3}\right]^{\frac{1}{2}} \\
\frac{\omega_{Q,-}}{A}=\frac{E\left(-3^{\prime}\right)-E\left(-2^{\prime}\right)}{A}= & 3(1-\eta)+3\left[(4+\eta)^{2}+\frac{5 \eta^{2}}{3}\right]^{\frac{1}{2}}
\end{aligned}
$$

where $A=\frac{e^{2} q Q\left({ }^{10} \mathrm{~B}\right)}{60 \hbar}$. The average of these two transitions is at

$$
\frac{\omega_{Q,{ }^{10} \mathrm{~B}}}{A}=6-3\left[1+\frac{5 \eta^{2}}{3}\right]^{\frac{1}{2}}+\frac{3}{2}\left\{\left[(4+\eta)^{2}+\frac{5 \eta^{2}}{3}\right]^{\frac{1}{2}}+\left[(4-\eta)^{2}+\frac{5 \eta^{2}}{3}\right]^{\frac{1}{2}}\right\}
$$

The asymmetry parameter $(\eta)$ and the electric field gradient $(e q)$ of a given nuclear site are expected to be the same for both ${ }^{10} \mathrm{~B}$ and ${ }^{11} \mathrm{~B}$ nuclei, while the strength of the interaction $\left(e^{2} q Q\right)$ will depend on the particular isotope involved [9]. When a ${ }^{10} \mathrm{~B}$ and ${ }^{11} \mathrm{~B}$ resonance has been observed, it becomes possible to determine the quadrupole interaction parameters. The value of $\eta$ can be calculated from the ratio $\frac{\omega_{Q, 10^{B}}}{\omega_{Q, 11 \mathrm{~B}}}$ since the ratio $\frac{Q\left({ }^{10} \mathrm{~B}\right)}{Q\left({ }^{11} \mathrm{~B}\right)}$ is known [108]. Then the quadrupole coupling constants can be determined, also using Equations 5.1 and 5.8.

The ${ }^{10} \mathrm{~B}$ transitions would normally not be observable using this SQUID technique due to the very small $I_{z}$ value of the ${ }^{10} \mathrm{~B}$ eigenstates, and consequently small 
longitudinal magnetization in low magnetic fields. The small $I_{z}$ value of the eigenstates arises because of the mixing of the states given by Creel. However, the $\pm 2^{\prime} \leftrightarrow \pm 3^{\prime}$ transitions of the ${ }^{10} \mathrm{~B}$ are observable in this case because they are close to the ${ }^{11} \mathrm{~B}$ resonance. Spin diffusion exists between the ${ }^{10} \mathrm{~B}$ and ${ }^{11} \mathrm{~B}$ spin systems allowing transitions within the ${ }^{10} \mathrm{~B}$ spin system to affect the longitudinal magnetization of the ${ }^{11} \mathrm{~B}$ system $[18,29,32]$. The spin diffusion mechanism is supported by the dependence of the shoulders' intensity on the direction of the rf sweep. The shoulder is barely visible during a rapid low to high frequency sweep, since the ${ }^{11} \mathrm{~B}$ spins have already been saturated. During a sweep from high to low frequency, the shoulder is more pronounced since the ${ }^{11} \mathrm{~B}$ spins have not been irradiated yet. We were unable to observe any of the lower frequency transitions of the ${ }^{10} \mathrm{~B}$ nuclei.

Various methods to better resolve the ${ }^{10} \mathrm{~B}$ and ${ }^{11} \mathrm{~B}$ resonances were attempted, such as lowering the small dc magnetic field, different degrees of isotopic enrichment, and deuteration of the hydrated compounds. None of these methods had a very strong effect on the resolution.

The resonance frequencies of the samples were determined by fitting each spectrum to the sum of two Gaussians. It is of interest to point out that the ${ }^{10} \mathrm{~B}$ resonances are about half as wide as the ${ }^{11} \mathrm{~B}$ resonances, because of the lower gyromagnetic ratio $(\gamma)$ and reduced dipolar coupling of ${ }^{10} \mathrm{~B}$. This reduced dipolar coupling of ${ }^{10} \mathrm{~B}$ is caused by the quenching of the dipolar interaction in integer spin systems [109]. The ${ }^{10} \mathrm{~B}$ and ${ }^{11} \mathrm{~B}$ resonance frequencies observed for the three compounds, along with the calculated quadrupolar parameters using Equations 5.1 and 5.8, are shown in Table 5.3. Butler and Brown used field-cycling to observe many of the ${ }^{10} \mathrm{~B}$ and ${ }^{11} \mathrm{~B}$ transitions in $\mathrm{B}_{2} \mathrm{O}_{3} \cdot 3 \mathrm{H}_{2} \mathrm{O}$ [100]. In their work, they used 


\begin{tabular}{|c|c|c|c|c|c|c|}
\hline Compound & Nucleus & $\begin{array}{c}\text { Resonance } \\
\text { Frequency } \\
(\mathrm{kHz})\end{array}$ & \multicolumn{2}{|c|}{$\begin{array}{c}\text { CRC } \\
Q\left({ }^{10} \mathrm{~B}\right) / Q\left({ }^{11} \mathrm{~B}\right) \text { ratio }\end{array}$} & $\begin{array}{c}\text { Butler } \\
Q\left({ }^{10} \mathrm{~B}\right) / Q \\
\frac{\mathrm{g}^{2} q Q\left({ }^{12} \mathrm{~B}\right)}{h} \\
(\mathrm{kHz})\end{array}$ & $\begin{array}{l}\text { rown } \\
\text { B) ratio } \\
\eta\end{array}$ \\
\hline \multirow[t]{2}{*}{$\mathrm{B}_{2} \mathrm{O}_{3} \cdot 3 \mathrm{H}_{2} \mathrm{O}$} & ${ }^{10} \mathrm{~B}$ & $1332 \pm 4$ & 2565 & 0.16 & 2571 & 0.11 \\
\hline & ${ }^{11} \mathrm{~B}$ & $1288 \pm 2$ & \pm 6 & \pm 0.06 & \pm 6 & \pm 0.11 \\
\hline \multirow[t]{2}{*}{$\mathrm{B}_{2} \mathrm{O}_{3} \cdot \mathrm{H}_{2} \mathrm{O}$} & ${ }^{10} \mathrm{~B}$ & $1367 \pm 5$ & 2644 & 0.25 & 2651 & 0.22 \\
\hline & ${ }^{11} \mathrm{~B}$ & $1336 \pm 2$ & \pm 7 & \pm 0.04 & \pm 8 & \pm 0.05 \\
\hline \multirow[t]{2}{*}{$\mathrm{B}_{2} \mathrm{O}_{3}$} & ${ }^{10} \mathrm{~B}$ & $1362 \pm 4$ & 2623 & 0.17 & 2630 & 0.12 \\
\hline & ${ }^{11} \mathrm{~B}$ & $1318 \pm 4$ & \pm 8 & \pm 0.08 & & \pm 0.12 \\
\hline
\end{tabular}

Table 5.3: Quadrupolar parameters calculated for the three borates, using both the ${ }^{10} \mathrm{~B}$ and ${ }^{11} \mathrm{~B}$ resonances, for two different values of the ratio of quadrupole moments. The $\frac{e^{2} q Q}{h}$ calculated is for ${ }^{11} \mathrm{~B}$. 
these transitions to determine the quadrupolar parameters of the boron nuclei in addition to the ratio $\frac{Q\left({ }^{10} \mathrm{~B}\right)}{Q\left({ }^{11} \mathrm{~B}\right)}$. Their ratio of 2.075 deviates from the accepted value of 2.084 [108]. Utilizing the ${ }^{11} \mathrm{~B}$ resonance and only the $\pm 2^{\prime} \leftrightarrow \pm 3^{\prime}$ transitions of ${ }^{10} \mathrm{~B}$ from the Butler and Brown work, along with Equations 5.1 and 5.8, the quadrupolar parameters can be determined and the results from their data is presented in Table 5.4. Comparison of Tables 5.3 and 5.4 shows that our result for $\mathrm{B}_{2} \mathrm{O}_{3} \cdot 3 \mathrm{H}_{2} \mathrm{O}$ agrees well with the work of Butler and Brown.

The relatively low asymmetry parameter calculated for $\mathrm{B}_{2} \mathrm{O}_{3} \cdot 3 \mathrm{H}_{2} \mathrm{O}$ is in agreement with the fact that each boron nucleus is surrounded by an equilateral triangle of hydroxyl groups [103]. Since the structure of $\mathrm{B}_{2} \mathrm{O}_{3} \cdot \mathrm{H}_{2} \mathrm{O}$ is similar to $\mathrm{B}_{2} \mathrm{O}_{3} \cdot 3 \mathrm{H}_{2} \mathrm{O}$ except for the lower extent of hydrogen bonding, this increases the deviation of the boron site from $\mathrm{C}_{3 V}$ symmetry. This structural change is evident in a slight increase of the asymmetry parameter. The relatively low asymmetry parameter for crystalline $\mathrm{B}_{2} \mathrm{O}_{3}$ is also in agreement with its structure which consists of essentially trigonal $\mathrm{BO}_{3}$ groups. Our results agree reasonably well with other works but is unable to resolve the issue of different values for the ratio of quadrupole moments. The different ratios of quadrupole moments may arise from a slight change in the electric field gradients at the nuclear site due to the isotope effect's influence on the equilibrium position of the nucleus.

\subsection{Boron Oxide Glass, $\mathrm{B}_{2} \mathrm{O}_{3}$}

$\mathrm{B}_{2} \mathrm{O}_{3}$ also exists in the glass phase which has been studied by magnetic resonance $[28,89,97,99,101,110]$. These studies, however, did not give much important information such as linewidths of resonances, a measure of the randomness 


\begin{tabular}{|c|c|c|c|c|c|c|}
\hline Compound & Nucleus & $\begin{array}{c}\text { Resonance } \\
\text { Frequency } \\
\quad(\mathrm{kHz})\end{array}$ & $\begin{array}{c}\text { CRC } \\
Q\left({ }^{10} \mathrm{~B}\right) / Q\left({ }^{11} \mathrm{~B}\right) \text { ratio }\end{array}$ & $\begin{array}{c}\text { B) ratio } \\
\eta\end{array}$ & $\begin{array}{c}\text { Butler } \\
Q\left({ }^{10} \mathrm{~B}\right) / Q \\
\frac{\left.\mathrm{e}^{2} q Q Q^{11} \mathrm{~B}\right)}{h} \\
(\mathrm{kHz})\end{array}$ & $\begin{array}{l}\text { rown } \\
\text { B) ratio } \\
\eta\end{array}$ \\
\hline \multirow[t]{2}{*}{$\mathrm{B}_{2} \mathrm{O}_{3} \cdot 3 \mathrm{H}_{2} \mathrm{O}$} & ${ }^{10} \mathrm{~B}$ & $1335 \pm 1$ & 2568 & 0.14 & 2575 & 0.06 \\
\hline & ${ }^{11} \mathrm{~B}$ & $1288 \pm 4$ & \pm 5 & \pm 0.07 & \pm 7 & \pm 0.06 \\
\hline
\end{tabular}

Table 5.4: Quadrupolar parameters calculated for $\mathrm{B}_{2} \mathrm{O}_{3} \cdot 3 \mathrm{H}_{2} \mathrm{O}$, for the two different quadrupole moment ratios, using on'y the $\pm 2^{\prime} \leftrightarrow \pm 3^{\prime}{ }^{10} \mathrm{~B}$ transitions and $\pm \frac{1}{2} \leftrightarrow \pm \frac{3}{2}{ }^{11} \mathrm{~B}$ resonance from the work of Butler and Brown. The $\frac{e^{2} q Q}{h}$ calculated is for ${ }^{11} \mathrm{~B}$. 
of the system and distribution in quadrupolar sites. A distribution of sites will result in a distribution of resonance frequencies and lead to a broader resonance.

The $\mathrm{B}_{2} \mathrm{O}_{2}$ glass sample was made by heating polycrystalline $\mathrm{B}_{2} \mathrm{O}_{3}$ (Aldrich, $99 \%$ purity) in a graphite mold. A propane/oxygen flame was applied for about 30 minutes; and by thrir all the bubbling within the melt had stopped. The sample was slowly cooled to room temperature and extracted from the graphite mold, resulting in a glass slug $4 \mathrm{~mm}$ in ủizmeter and about $1 \mathrm{~cm}$ long. Figure 5.7 shows the spectrum of this sample. The ${ }^{11} \mathrm{~B}$ resonance frequency was determined to be $1360 \pm 10 \mathrm{kHz}$ which is in excellent agreement with other works $\left[2^{7}, 99,101\right]$. This figure illustrates the necessity and advantages of using forward and reverse sweeps to obtain the final spectrum. Using a forward minus reverse sweep allows one to symmetrize the overall resonance and to neglect the effects of cross-relaxation and spin-lattice relaxation on the lineshape. As cen be seen from the single sweep direction spectra, using only a single sweep direction spectrum to determine the resonance frcquency would lead to an erroneous resonance frequency reading. Secondly, the response time of the entire system does not influence the final spectrum. And finally, instrumental artifacts, such as the one near $1100 \mathrm{kHz}$, are averaged away by subtracting the reverse sweep from the forward sweep.

The linewidth of the $\mathrm{B}_{2} \mathrm{O}_{3}$ glass resonance was determined to be around $80 \mathrm{kHz}$ (FWHM). This large linewidth is consistent with glassy or amorphous substances where a distruibution in quadrupolar sites is expected. The linewidth of this spectrum is dominated by the distribution in sites, as the applied magnetic field was only about $3 \mathrm{G}$. This is a prime example of a case where the NQR resonance obtained using the SQUID technique very closely approximates the conventional NQR 


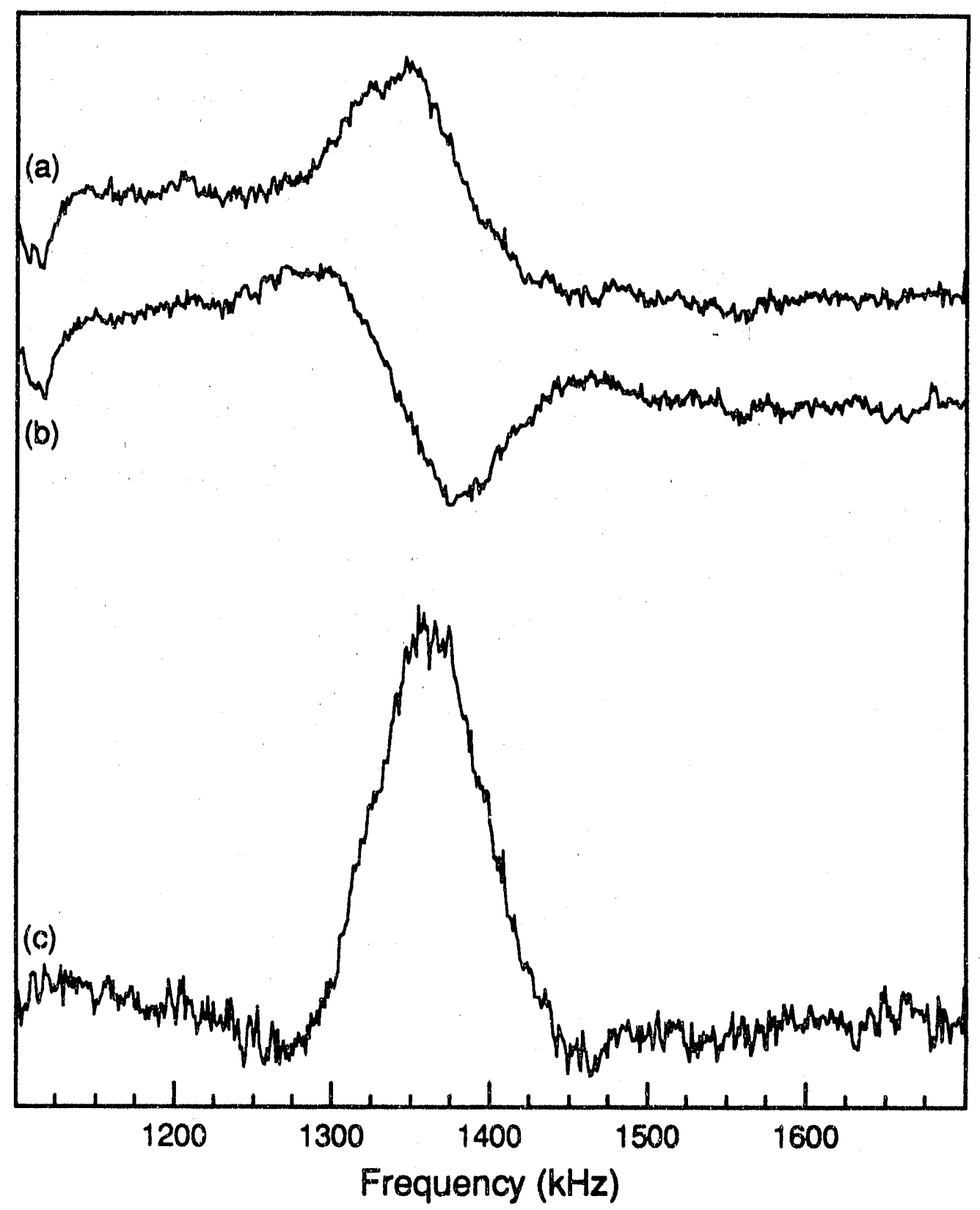

Figure 5.7: ${ }^{11} \mathrm{~B}$ spectra of boron oxide $\left(\mathrm{B}_{2} \mathrm{O}_{3}\right)$ glass: (a)forward sweeps, (b)reverse sweeps, and (c)forward-reverse sweeps. The dc field was about $3 \mathrm{G}$ and the instrumental artifact near $1100 \mathrm{kHz}((\mathrm{a})$ and (b)) is averaged away by subtracting the reverse sweeps from the forward sweeps to get the final spectrum, (c). The large linewidth of the resonance is characteristic of amorphous materials where there is a large distribution of sites. 
lineshape and linewidth. Previous simulations of high-field spectra have yielded standard deviation estimates of $\sigma\left(\frac{e^{2} q Q}{h}\right)=100 \mathrm{kHz}$ and $\sigma(\eta)=0.043$ [99]. Recently, Bray's group used a conventional cw NQR spectrometer to detect this resonance and obtained a linewidth of $60 \mathrm{kHz}[111,112,113]$.

As noted in Chapter 2, since ${ }^{11} \mathrm{~B}$ has spin $I=\frac{3}{2}$ there is no way to extract $\frac{e^{2} q Q}{h}$ and $\eta$ from the single resonance frequency. This also does not allow us to extract independent measures of the variations in those two quantities. One may ask, "Is it possible to use the ${ }^{10} \mathrm{~B}$ resonance to get $\frac{\mathrm{e}^{2} q Q}{h}$ and $\eta$ as wis done in the previous section on $\mathrm{B}_{2} \mathrm{O}_{3} \cdot \mathrm{xH}_{2} \mathrm{O}$ ?" In theory, yes. In practice for this case, no. This is because we do not observe any shoulders in this glass spectrum, so the ${ }^{10} \mathrm{~B}$ resonance is either completely underneath the wide ${ }^{11} \mathrm{~B}$ resonance or too far away from the main resonace. Previous work on the glass phase of ${ }^{0} \mathrm{~B}$-enriched $\mathrm{B}_{2} \mathrm{O}_{3}$ shows the $\pm 2^{\prime} \leftrightarrow \pm 3^{\prime}{ }^{10} \mathrm{~B}$ transitions to be around $1375 \mathrm{kHz}[27,99]$. This result along with ours gives $\eta=0.33$, using the accepted value for the ratio $\frac{\left.Q^{(10} B\right)}{\left.Q{ }^{11} B\right)}$.

An intriguing prospect is to conduct some classic hole-burning experiments [114] in the $\mathrm{B}_{2} \mathrm{O}_{3}$ glass. If irradiating the sample with a constant frequency rf causes the entire resonance to disappear or lower in amplitude, then one can say that the distribution of sites is homogeneous throughout the entire sample. On the other hand, one may burn a "hole" in the spectrum. This would indicate that domains of similar sites exist in the sample and communication (spin-diffusion) between like domains is hindered by many domains of un-like sites. Another prospect is to study lithium-boron glasses, sodium-boron glasses, and other alkali-metal boron glasses [115]. This would allow one to determine how the ${ }^{11} \mathrm{~B}$ resonance frequency changes with varying composition and varying amounts of these alkali metals. 


\section{Chapter 6}

\section{$I=\frac{\mathbf{5}}{\mathbf{2}} \mathrm{NQR}$ Results}

\subsection{Introduction}

Spin $I=\frac{5}{2}$ nuclei include useful 8 nd important nuclei such as ${ }^{27} \mathrm{Al},{ }^{17} \mathrm{O}$, and many others. High natural abundance nuclei, such as ${ }^{27} \mathrm{Al}$, are found in technologically important materials such as zeolites. On the other hand, the natural bundance of ${ }^{17} \mathrm{O}$ is very low. ${ }^{17} \mathrm{O}$-enriched compounds, however, can be synthesized and then studied by NQR. Since spin $I=\frac{5}{2}$ nuclei give two pure NQR resonances, the quadrupole coupling constant $\left(\frac{e^{2} q Q}{h}\right)$ and asymmetry parameter $(\eta)$ can be determined. Once both of the quadrupolar parameters have been determined, it is possible to perform some Quantum Mechanics calculations and extract more detailed information on the structure of the material. For this reason, materials containing spin $I=\frac{5}{2}$ nuclei tend to be able to give more dynamical information on the material, whether one is interested in the material itself (i.e. phase transitions) or in the material as it undergoes a chemical reaction. It is for these reasons that nuclear quadrupole resonance studies of compounds containing spin $I=\frac{5}{2}$ nuclei tend to give more 
structural and dynamical information than those containing $I=\frac{3}{2}$ nuclei.

This chapter concentrates heavily on the study of the $\alpha-\mathrm{Al}_{2} \mathrm{O}_{3}$ single crystal and powder samples which were used to confirm and develop the theory in Chapter 2. $\alpha-\mathrm{Al}_{2} \mathrm{O}_{3}$ was an excellent sample to do this study due to the availability of high quality crystals, simple crystal structure, good signal-to-noise ratio, and convenient $T_{1}$ value. An $\alpha-\mathrm{Al}_{2} \mathrm{O}_{3}$ single crystal was also used by Jach to introduce this SQUID NQR technique [25] .

\subsection{Sapphire, $\alpha-\mathrm{Al}_{2} \mathrm{O}_{3}$}

\subsubsection{Single Crystal Sample}

$\mathrm{Al}_{2} \mathrm{O}_{3}$ (alumina) exists in three forms referred to as the $\alpha, \beta$, and $\gamma$ forms [88]. The $\alpha$ form, the most stable form of $\mathrm{Al}_{2} \mathrm{O}_{3}$, is the phase which was used throughout this chapter. The $\beta$ and $\gamma$ forms of $\mathrm{Al}_{2} \mathrm{O}_{3}$ are in general not highly ordered, and therefore not suitable as test samples. We attempted to observe signal from $\gamma-\mathrm{Al}_{2} \mathrm{O}_{3}$ but were unsuccessful.

$\alpha-\mathrm{Al}_{2} \mathrm{O}_{3}$ (sapphire, corundum) is a very highly-ordered system and very dense at $4 \mathrm{~g} / \mathrm{ml}$. The packing scheme of the oxygen atoms of $\alpha-\mathrm{Al}_{2} \mathrm{O}_{3}$ is hexagonal closest packing which makes the aluminum nuclei sit in a distorted octahedron formed by six oxygen atoms [116], Figure 6.1. Two-thirds of all the octahedral sites are filled by aluminum in a well-defined fashion. It may be of interest to point out that if the octahedron formed by the oxygen nuclei were perfect, the extremely high symmetry of the site would make the quadrupolar interaction vanish [9]. The distorted octahedral site has the aluminum equally displaced between the two equilateral 


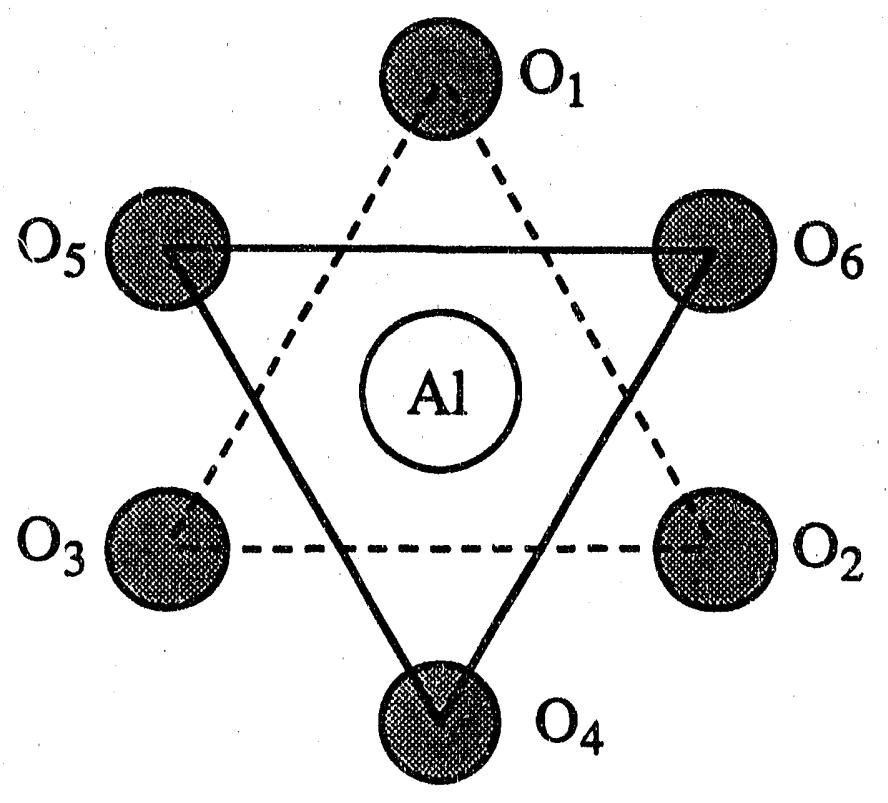

Figure 6.1: The ${ }^{27} \mathrm{Al}$ nucleus in $\alpha-\mathrm{Al}_{2} \mathrm{O}_{3}$ is sandwiched between two equilateral triangles of oxygen atoms. Oxygen nuclei $\mathrm{O}_{1}, \mathrm{O}_{2}$, and $\mathrm{O}_{3}$ form one triangle while $\mathrm{O}_{4}, \mathrm{O}_{5}$, and $\mathrm{O}_{6}$ form the other triangle. The z-axis of the principal axis system is perpendicular to the page and penetrates the ${ }^{27} \mathrm{Al}$ nucleus. 
triangles of oxygen atoms on top and below. These two triangles of oxygen atoms are rotated by $60^{\circ}$ with respect to each other. This makes the space group of the site $\mathrm{D}_{3 d}$, where the z-axis of the quadrupolar interaction is the main symmetry $\left(\mathrm{C}_{3}\right)$ axis $[117,118]$. Later, we show that $\eta=0.00 \pm 0.06$, but for now, symmetry considerations make the asymmetry parameter very close to zero.

The single crystal of $\alpha-\mathrm{Al}_{2} \mathrm{O}_{3}$ was a colorless cylinder of 0.25 " in length and 0.125 " in diameter. It was purchased from Union Carbide and had a stated maximum paramagnetic impurity cortent of $2 \mathrm{ppm}$ [119]. The cylinder was cut such that the cylindrical axis was aligned with the c-axis of the unit cell, which is also the z-axis of the PAS. The powder sample was made by crushing similar single crystals obtained from Union Carbide. These other crystals, used to get the powder, were also colorless but not from the same parent crystal, so no serious attempt should be made to correlate relaxation times which depend on purity.

The spectrum of $\alpha-\mathrm{Al}_{2} \mathrm{O}_{3}$ single crystal is shown in Figure 6.2. The NQR resonance frequencies, using forward-reverse sweeps, for the $\pm \frac{1}{2} \leftrightarrow \pm \frac{3}{2}$ and $\pm \frac{3}{2} \leftrightarrow \pm \frac{5}{2}$ resonances were determined to be $357 \pm 2 \mathrm{kHz}$ and $714 \pm 2 \mathrm{kHz}$ respectively. This is in excellent agreement with room temperature work by Pound and others $[1,25,120,121]$, meaning the resonance frequencies are temperature independent. Using Equations 2.39 and 2.40 the following parameters are calculated, $\frac{e^{2} q Q}{h}=2.38 \pm 0.01 \mathrm{MHz}$ and $\eta=0.00 \pm 0.06$.

Recently, Bray's group used their conventional NQR spectrometer to obtain the spectrum of $\alpha-\mathrm{Al}_{2} \mathrm{O}_{3}$ [8]. They determined the resonance frequencies to be 361.6 $\pm 0.4 \mathrm{kHz}$ and $715.5 \pm 0.7 \mathrm{kHz}$. This gives values of $\frac{e^{2} q Q}{h}=2.389 \pm 0.002 \mathrm{MHz}$ and $\eta=0.091 \pm 0.007$. The interesting part to note is that they used only a 

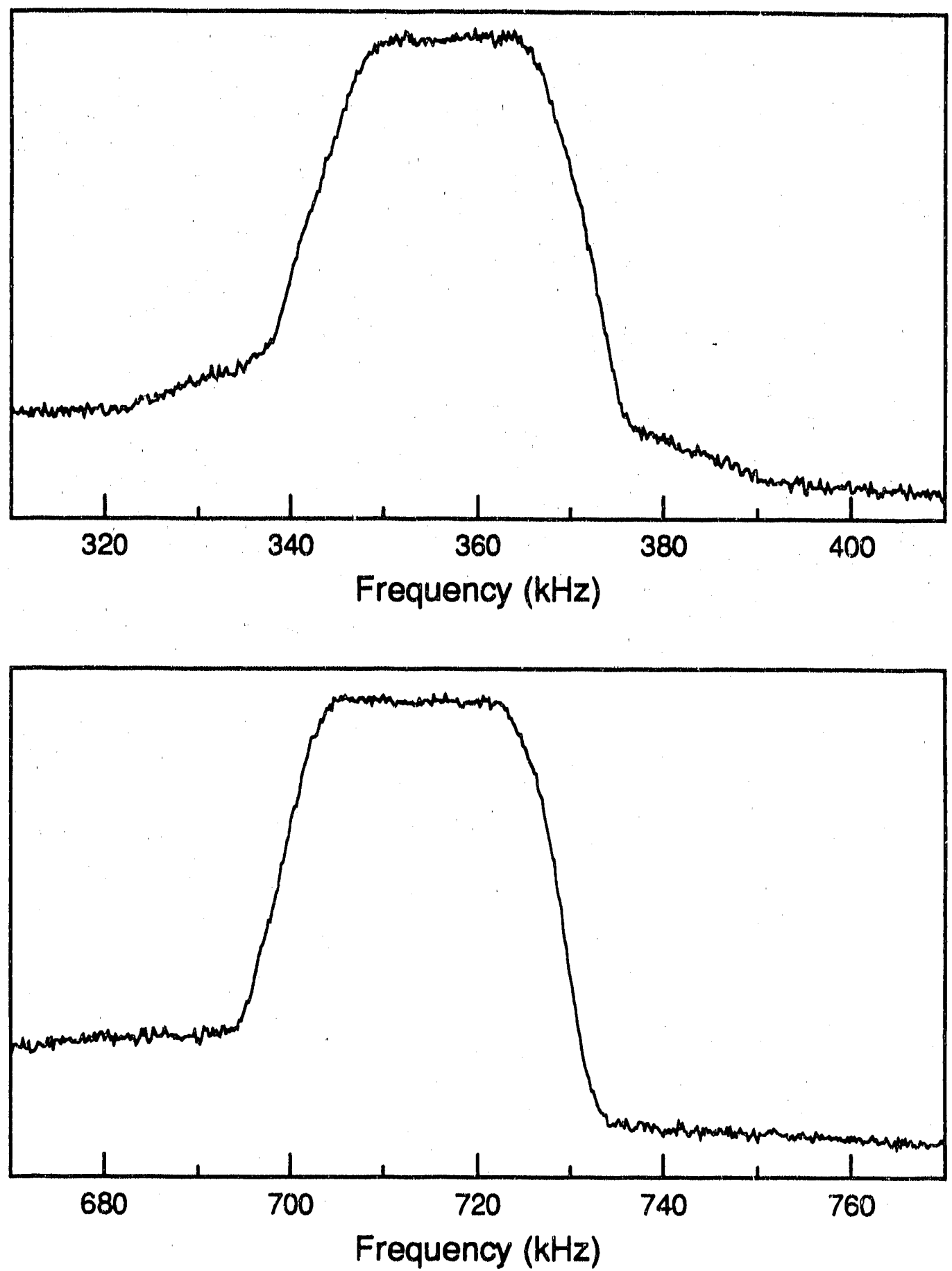

Figure 6.2: Low-frequency resonance $\left( \pm \frac{1}{2} \leftrightarrow \pm \frac{3}{2}\right.$ ) (top) and high-frequency resonance $\left( \pm \frac{3}{2} \leftrightarrow \pm \frac{5}{2}\right.$ ) (bottom) of ${ }^{27} \mathrm{Al}$ in a single crystal of $\alpha-\mathrm{Al}_{2} \mathrm{O}_{3}$. A dc magnetic field of about $28 \mathrm{G}$ was applied along the z-axis of the PAS. 
single sweep direction to determine these resonance frequencies. Comparing their resonance frequencies to ours, we get $\Delta \nu_{L}=+4.6 \mathrm{kHz}$ and $\Delta \nu_{H}=+1.5 \mathrm{kHz}$. However, they used a (sweep rate)/(time constant) ratio of $8.1 \mathrm{kHz} / \mathrm{TC}$ and 3.4 $\mathrm{kHz} / \mathrm{TC}$ for the low frequency and high frequency regions respectively. This makes the sweep width per time constant (TC) of their spectrometer about twice as large as the difference in resonance frequencies. Thus, the experimental conditions under which they obtained the spectrum seem to indicate that their spectrometer's long time constant influenced the exact frequency of these resonances.

Figure 6.3 shows the complete spectrum of the single crystal using only a forward sweep. The calculated signal magnitude, assuming a saturating rf field, is 0.23 $\mu \mathrm{G}$. The measured magnetization of the sample is $0.19 \mu \mathrm{G}$. Since the test coil, used to calibrate the magnetization, was larger than the sample, the actual sample magnetization was probably greater than $0.19 \mu \mathrm{G}$.

Detailed studies of the single crystal sample were conducted to confirm the theory of the experiment. A series of different forward sweep rates were used to acquire the spectra of the $\pm \frac{3}{2} \leftrightarrow \pm \frac{5}{2}$ resonance in Figure 6.4 to study lineshapes. The spectrum acquired with the fastest sweep rate closely resembles the plateau-shaped resonance described in Figure 2.3. Figure 6.4 should be compared to the simulations in Chapter 2, Figure 2.5. Figure 6.5 shows the simulations and experimental spectra together, and one can say that the model used for the simulations is quite good within the range of sweep rates used.

A confirmation of the theory and experiments of Weber and Hahn was attempted, to determine if we could observe the two components of a NQR resonance (i.e. $-\frac{3}{2} \leftrightarrow-\frac{5}{2}$ and $+\frac{3}{2} \leftrightarrow+\frac{5}{2}$ ) in a single crystal $[35,36]$. We applied 


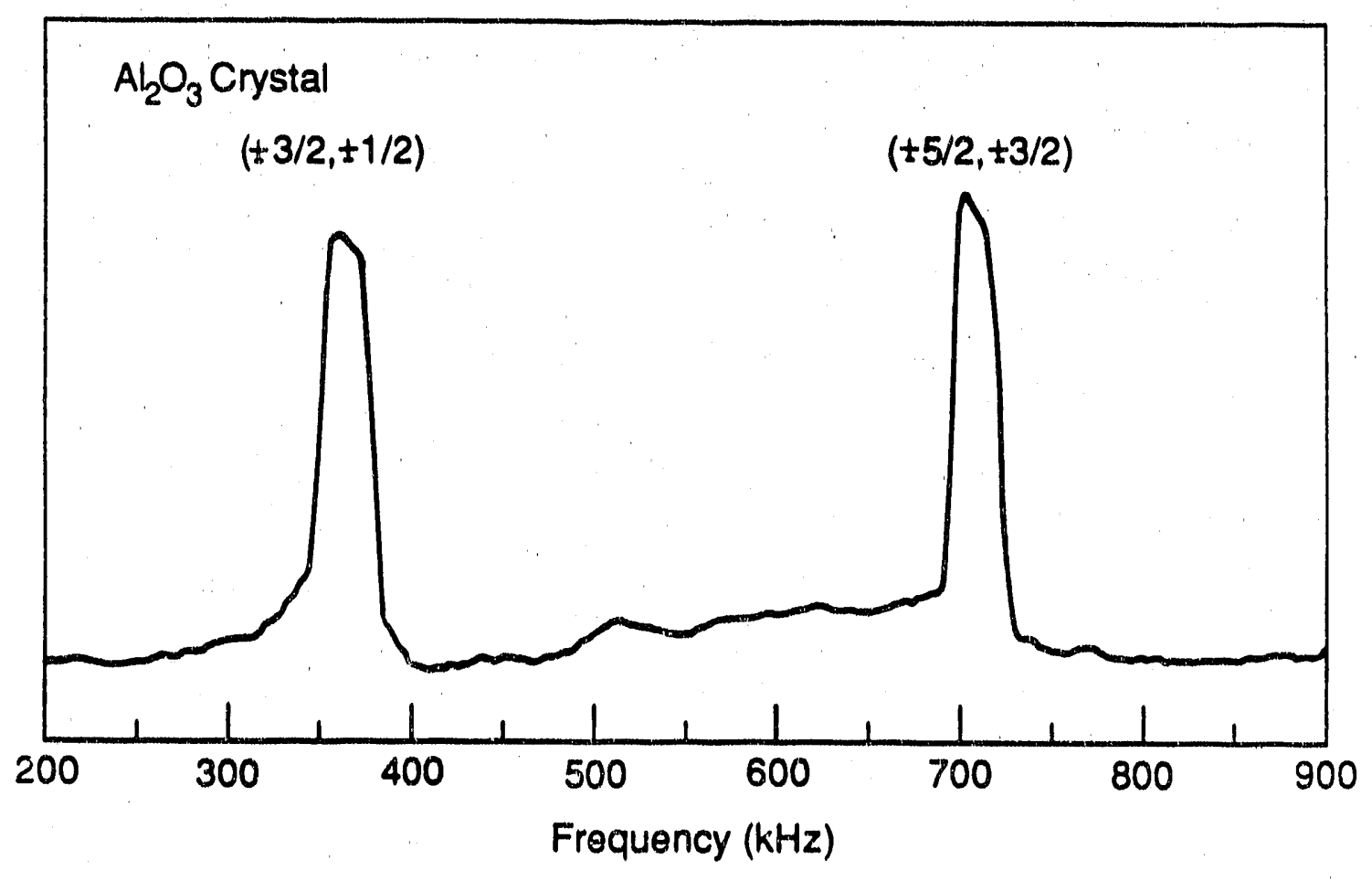

$X B L 8911-4300$

Figure 6.3: A complete sweep of the two resonances of a single crystal of $\alpha-\mathrm{Al}_{2} \mathrm{O}_{3}$ using only a forward sweep. The applied dc field was about $10 \mathrm{G}$. 


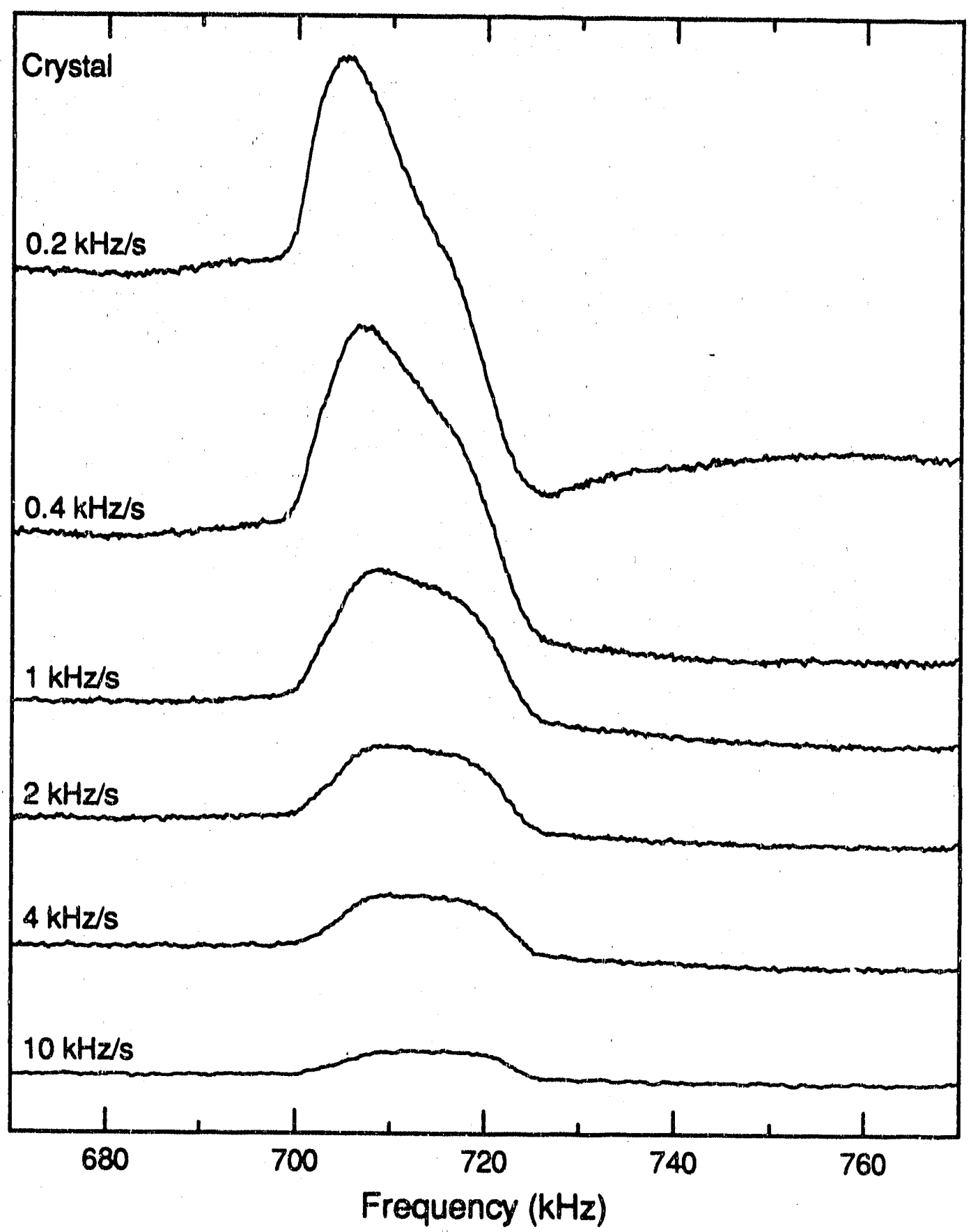

Figure 6.4: Forward sweeps of the $\pm \frac{3}{2} \leftrightarrow \pm \frac{5}{2}$ resonance of $\alpha-\mathrm{Al}_{2} \mathrm{O}_{3}$ single crystal, using various sweep rates. The signal-to-noise ratio was increased in some cases by averaging. However, the relative intensities of the peaks is normalized to the same number of sweeps. The applied dc field was about 10 G. 

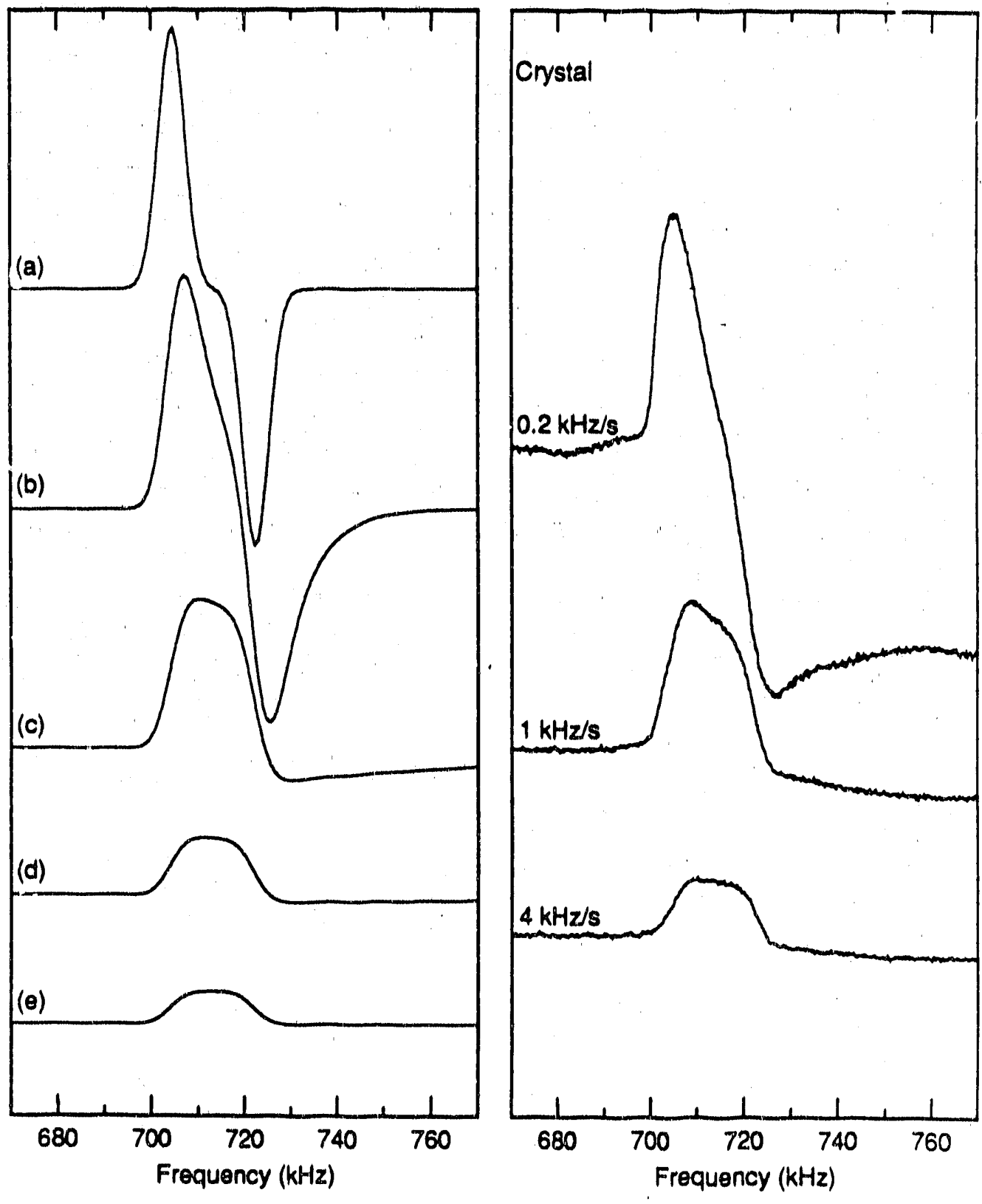

XBL $908-2801$

Figure 6.5: Simulated (left) and some experimental forward sweeps of the $\pm \frac{3}{2} \leftrightarrow \pm \frac{5}{2}$ resonance of the $\alpha-\mathrm{Al}_{2} \mathrm{O}_{3}$ single crystal. In Figures 6.5 and 6.12 , the amplitudes of the simulated spectra are matched to the amplitudes of the experimental spectra. The applied dc field was about $10 \mathrm{G}$. 
circularly polarized of to the resonance at $714 \mathrm{kHz}$ and the results are shown in Figure 6.6. The polarizatioas of the rf field are arbitrarily labelled. No serious attempt was made to correlate the polarization of the rf to a particular transition. The underlying theory to this figure is that once the rf has excited a transition, the magnetization of the sample will decay, back to its equilibrium value, with a time constant $T^{\prime}$. The sweeping of will not affect the other transition since it has the wrong polarization. If $T_{1}$ is much longer than $T_{C R}$, then $T^{\prime} \approx T_{C R}$. While if $T_{1} \approx T_{C R}$, the $T^{\prime}$ will be some complicated factor of $T_{1}$ and $T_{C R}$. The $T^{\prime \prime}$ determined for this case was $70 \pm 20 \mathrm{sec}$. The large uncertainty in this reading may be due to non-exponential relaxation [122]. This is plausible since we observed that $T_{1}$ was only slightly longer that $70 \mathrm{sec}$. Spin-lattice relaxation in rigid lattices is usually performed by the fluctuating dipole moments of paramagnetic impurities in the sample [123]. An approximate figure of $T_{1}=70 \mathrm{sec}$. is not inconsistent with previous measurements [122].

It is also possible to obtain an "absorption" spectrum of the overall NQR resonance. One method would be to use a very slow sweep rate. The method used here whs to first obtain the spectrum using a fast sweep, Figure 6.2. Then one takes the derivative of this spectrum, Figure 6.7. The widths of the two transitions are broadened by artificial Gaussian smoothing. In principle, the widths of these transitions is determined by the dipolar coupling between ${ }^{27} \mathrm{Al}$ nuclei $[122,124]$.

In order to simulate the powder lineshapes and also choose sweep parameters for samples that have not been previously examined, it is of some importance to look at how signal intensity depends on the rf field strength, magnetic field strength, and sweep rate. The single crystal of $\alpha-\mathrm{Al}_{2} \mathrm{O}_{3}$ was used to give us some preliminary 


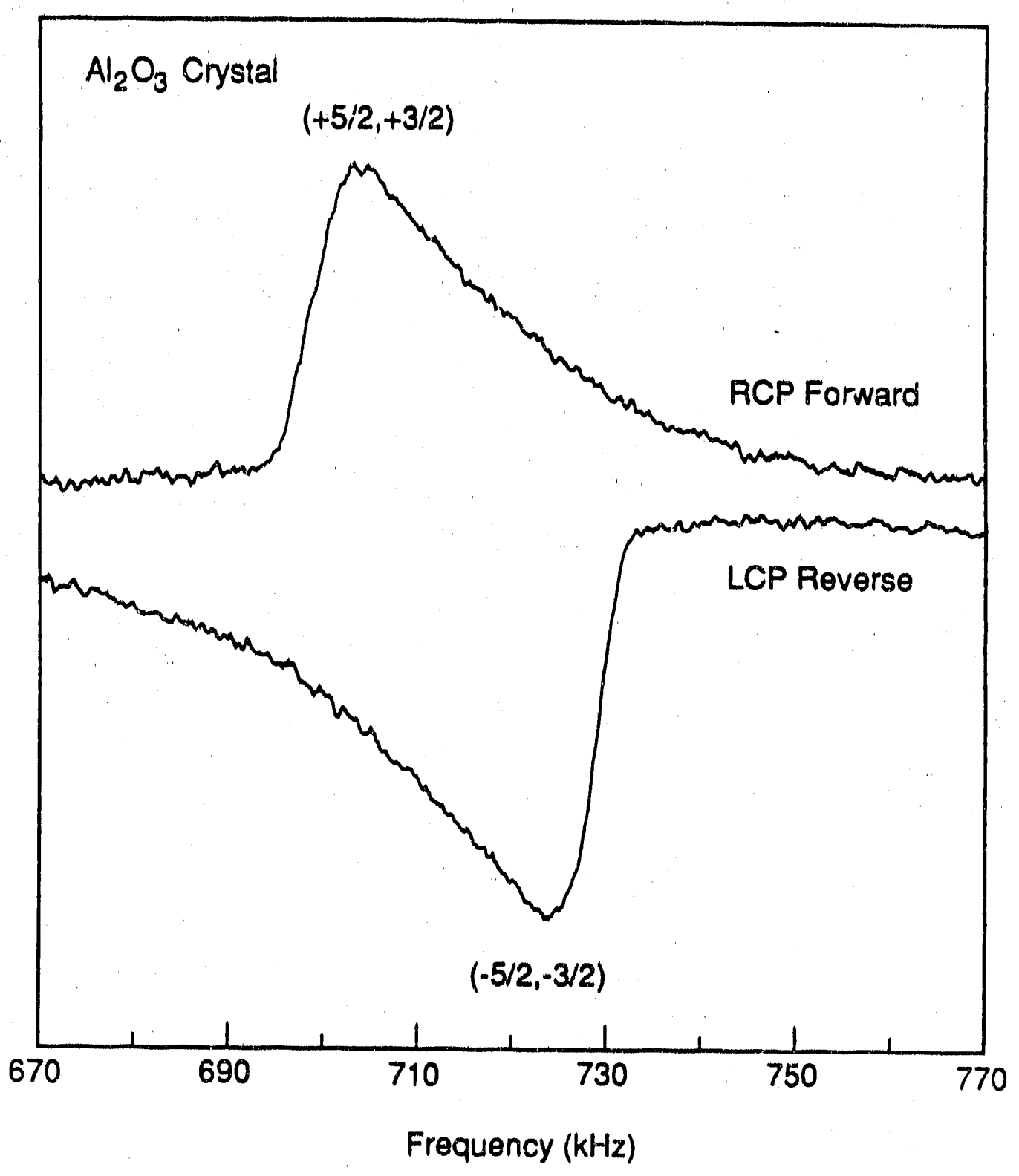

XEL 8911-4302

Figure 6.6: Forward (top trace) and reverse (bottom trace) sweeps of the $\alpha$ $\mathrm{Al}_{2} \mathrm{O}_{3}$ single crystal using of of opposite circular polarizations. The figure shows the two components of the overall resonance. The polarizations of the rf are arbitrarily labelled. In each sweep, the signal decays back to the thermal equilibrium magnetization, after each transition component, with a time constant $T^{\prime}$ which depends on $T_{1}$ and $T_{C R}$. 


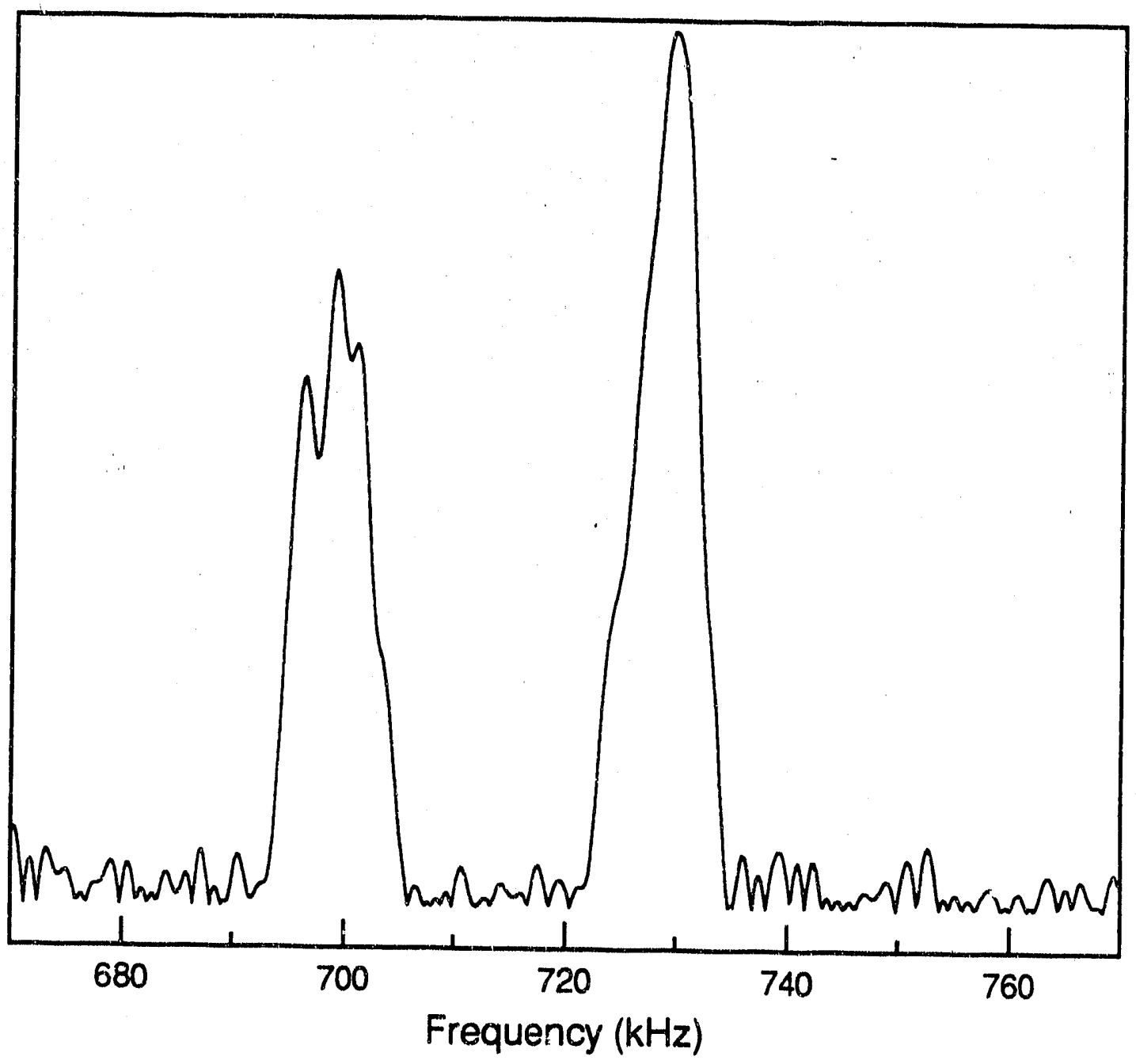

Figure 6.7: An "absorption" spectrum of the $\pm \frac{3}{2} \leftrightarrow \pm \frac{5}{2}$ resonance of the $\alpha$ $\mathrm{Al}_{2} \mathrm{O}_{3}$ crystal, revealing the two components of the overall resonance. This figure was generated from Figure 6.2, convoluting it with a Gaussian, differentiating it, and then taking the absolute value. 
data on this.

The plot of signal intensity versus rf strength is shown in Figure 6.8. The general shape of the curve can be described $b_{y}$ the expression $1-e^{-\frac{B_{1}}{B_{1,0}}}$, where $B_{1,0}$ is taken to be near the corner of the curve. In general $B_{1,0}$ depends on the gyromagnetic ratio of the nuclei and on the relaxation time, probably $T_{C R}$. Noting that the noise level of the SQUID output is somewhat proportional to rf strength, there will be some optimum value of $\mathrm{rf}$ strength that maximizes signal-to-noise. However, this optimum value is in general hard to predict a priori since $T_{C R}$ is difficult to predict at these temperatures and magnetic field strengths. One usually has to resort to determining this optimum value empirically. Although a plot of signal intensity versus magnetic field strength is not shown, we found that the expression $1-e^{-\frac{B_{0}}{\theta_{L}}}$ did approximate its behavior.

A plot of signal intensity versus sweep rate is shown in Figure 6.9. A qualitative description is based on a delicate balance of the amount of rf used in exciting transitions and the amount of time that relaxation is allowed to occur during the sweep. When one sweeps very fast, only a little rf is available for exciting transitions and not much time is allowed for relaxation to occur. This results in a small, but plateau-shaped, resonance. Although the opposite case of a very slow sweep is not depict ed in the figure, the behavior is predictable. In this case, much rf is available for the transitions but this is countered by lots of time for relaxation to occur. This results in a moderately high signal intensity, but the resonance lineshape is very asymmetrical. Again, there is some optimum sweep rate which results in a very good signal intensity and where not much time is allowed for relaxation processes. This results in an overall lineshape that has relatively good symmetry and signal 


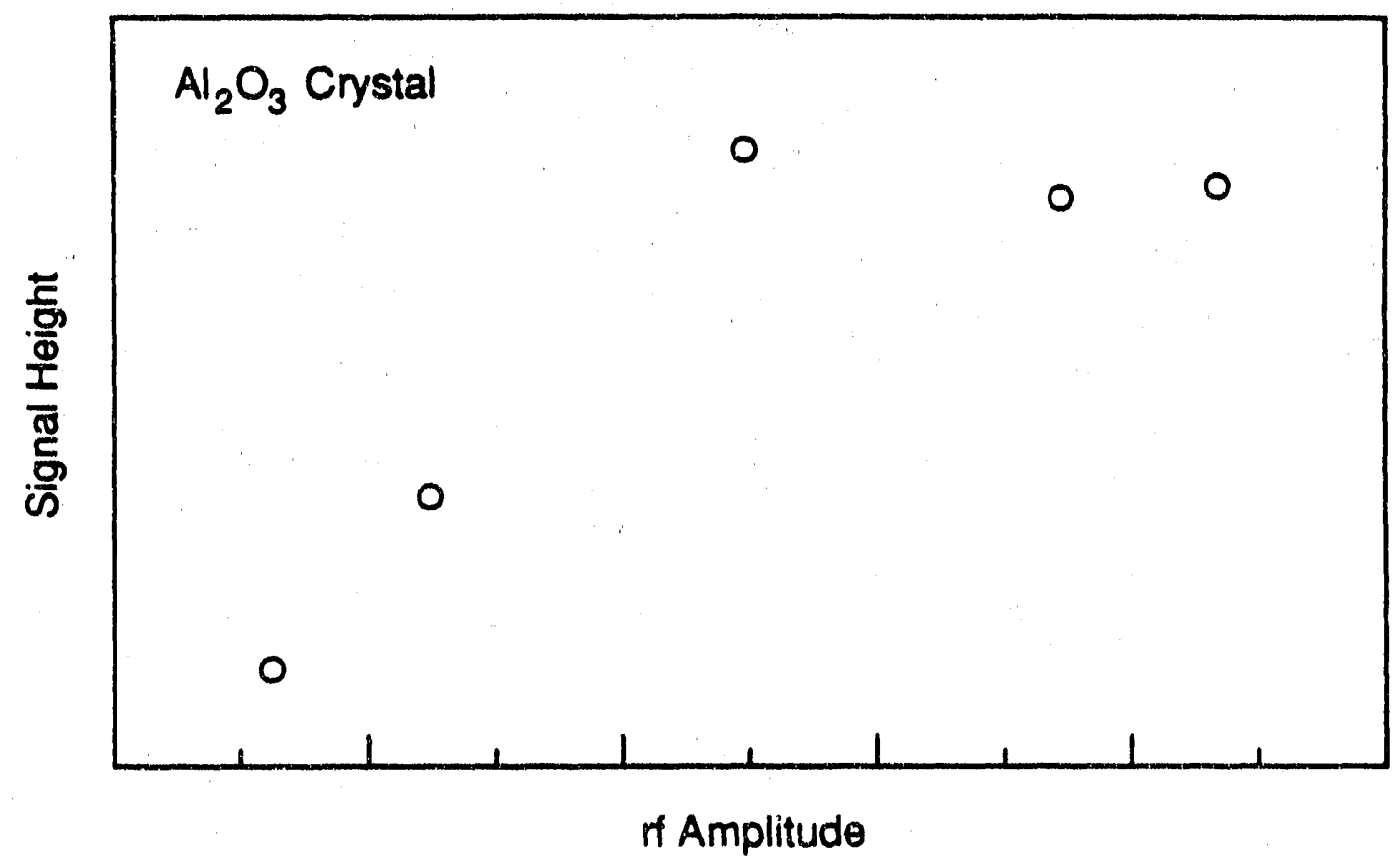

Figure 6.8: A plot of the signal amplitude as a function of the rf strength. A single crystal of $\alpha-\mathrm{Al}_{2} \mathrm{O}_{3}$ was used for these measurements. The resonance used was the $\pm \frac{3}{2} \leftrightarrow \pm \frac{5}{2}$ resonance and the rf was linearly polarized. 


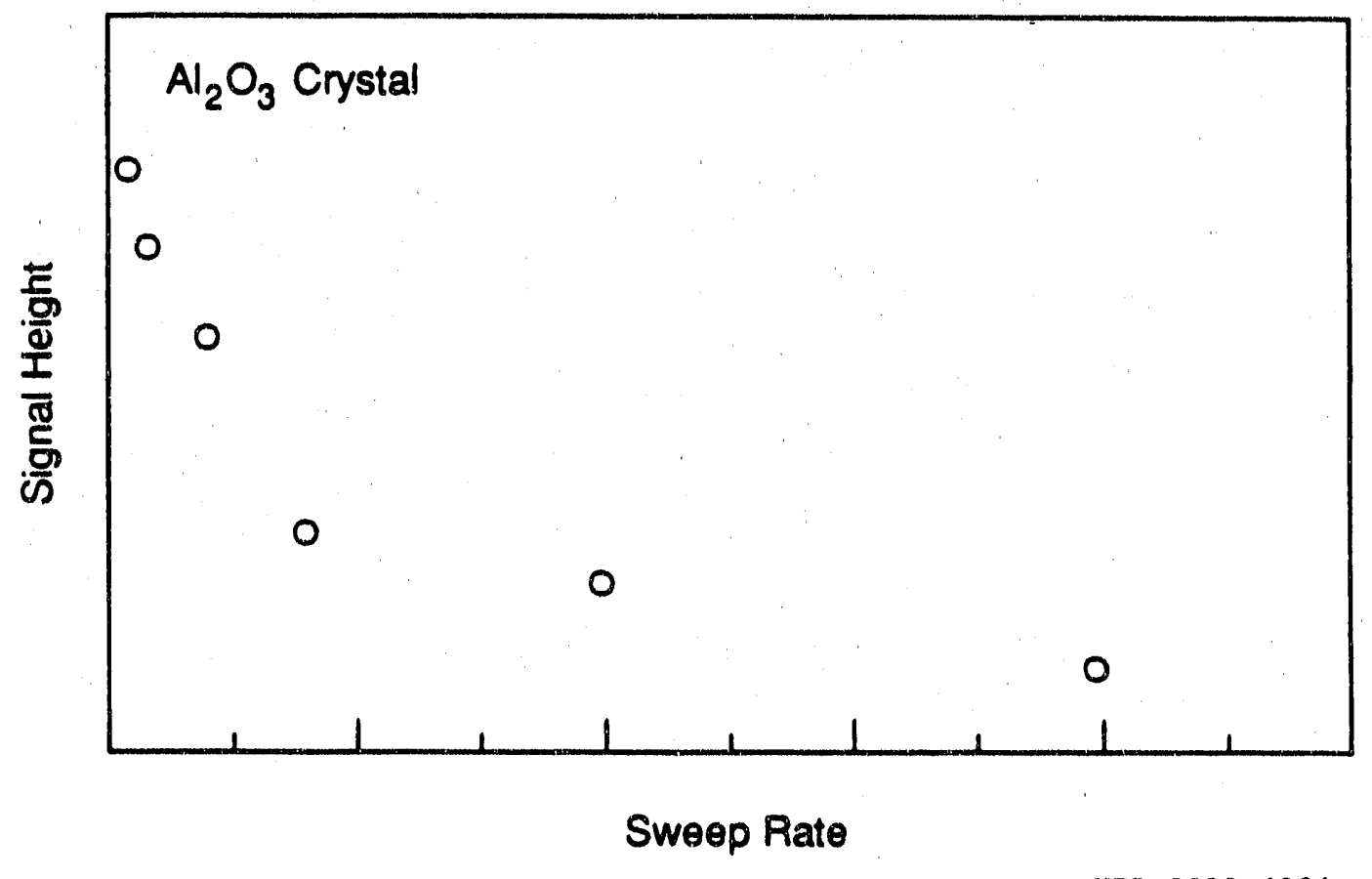

XBL $8911-4304$

Figure 6.9: A plot of the signal amplitude as a function of the sweep rate of the linearly polarized $\mathrm{rf}$. The $\pm \frac{3}{2} \leftrightarrow \pm \frac{5}{2}$ resonance of a $\alpha-\mathrm{Al}_{2} \mathrm{O}_{3}$ single crystal was used for these measurements. 
intensity.

Before proceeding to the $\alpha-\mathrm{Al}_{2} \mathrm{O}_{3}$ powder sample, one small test was necessary. For this SQUID technique to work for powder samples, the crystal had to give a signal of the same sign independent of whether it was right side up or upside down. In the original orientation, a forward sweep gave, say, a positive signal. In the other orientation, a forward sweep also gave a positive signal. If the second signal had been a negative one, this technique could not have been applied to powder samples.

\subsubsection{Powder Sample}

The last results on the $\alpha-\mathrm{Al}_{2} \mathrm{O}_{3}$ sample were on the crushed, powdered sample, Figure 6.10. The resonance frequencies and quadrupolar parameters for the powder sample were the same as those determined for the single crystal sample. Our ability to obtain this spectrum was the first experimental confirmation that this SQUID NQR technique would be applicable to powder samples. The signal amplitude of the powder sample was about $15 \%$ of the signal intensity for the single crystal.

Figure 6.11 shows a series of forward sweep spectra of the powdered sample for different sweep rates on the $\pm \frac{3}{2} \leftrightarrow \pm \frac{5}{2}$ resonance. This should be compared to the simulations of the powder lineshapes in Figure 2.6. Figure 6.12 shows the simulations and experimental together. A comparison can also be made between Figure 6.5 and Figure 6.12 .

As noted in Chapter 2, the $\pm \frac{3}{2} \leftrightarrow \pm \frac{5}{2}$ resonance for a powder sample can have a smaller linewidth than the same resonance of a single crystal sample. Figures 6.2 and 6.10 illustrate this point. On the other hand, also noted in Chapter 2, the $\pm \frac{1}{2} \leftrightarrow \pm \frac{3}{2}$ resonance usually has a larger linewidth than the $\pm \frac{3}{2} \leftrightarrow \pm \frac{5}{2}$ resonance 

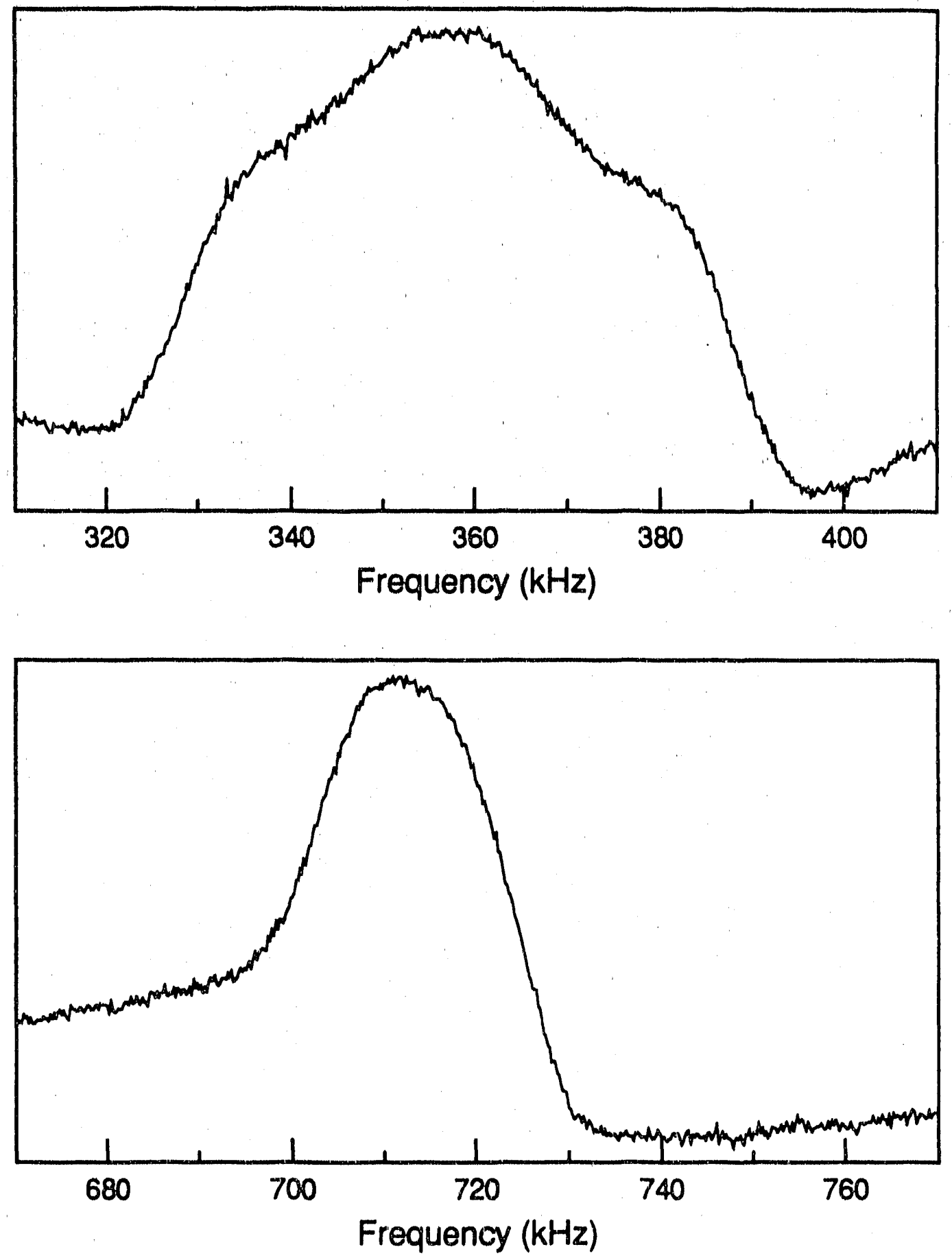

Figure 6.10: Low-frequency resonance $\left( \pm \frac{1}{2} \leftrightarrow \pm \frac{3}{2}\right.$ ) (top) and high-frequency resonance $\left( \pm \frac{3}{2} \leftrightarrow \pm \frac{5}{2}\right.$ ) (bottom) of ${ }^{27} \mathrm{Al}$ in powdered $\alpha-\mathrm{Al}_{2} \mathrm{O}_{3}$. The dc field used was the same as in Figure 6.2, about 28 G. 


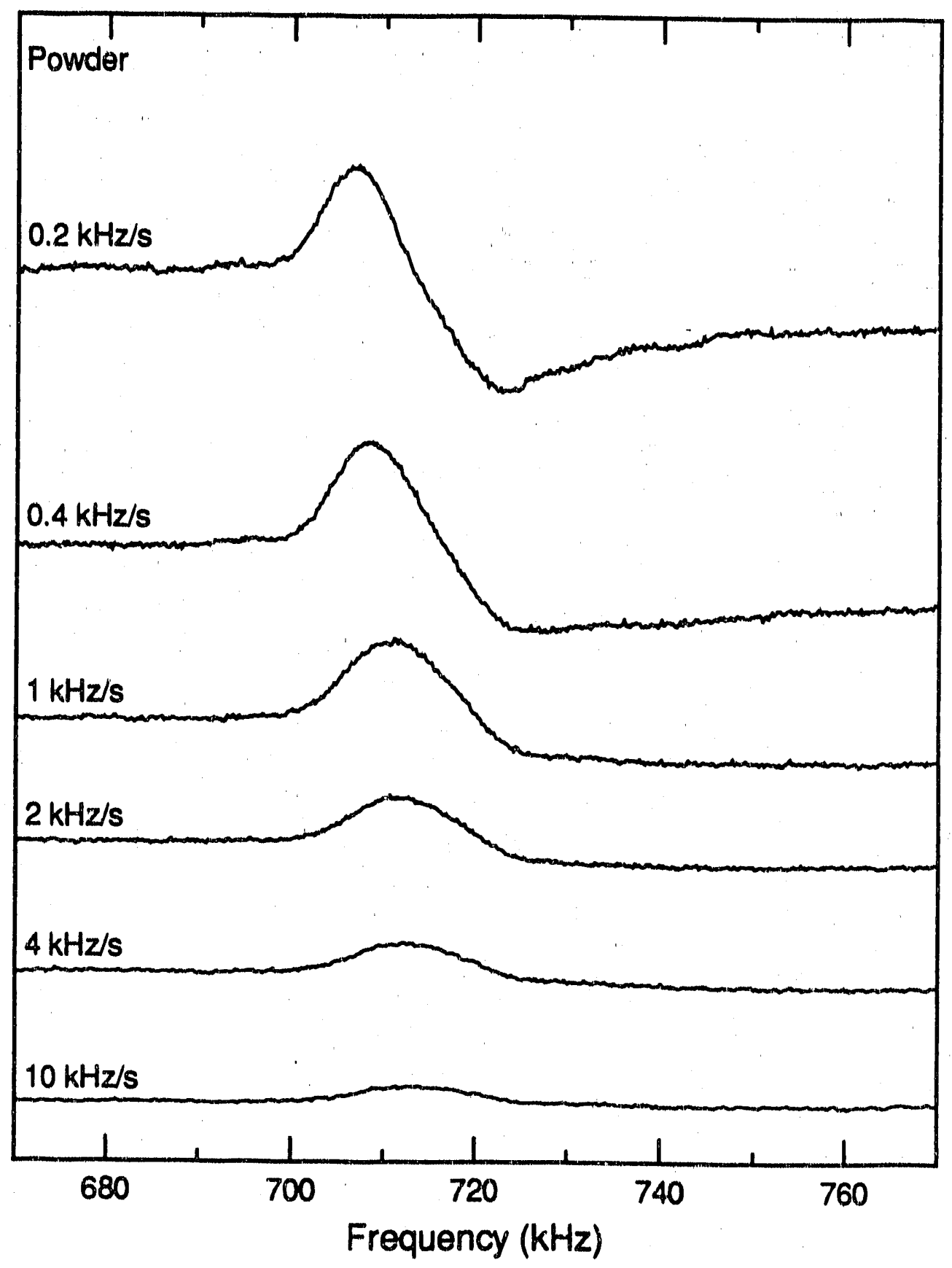

Figure 6.11: Forward sweeps of the $\pm \frac{3}{2} \leftrightarrow \pm \frac{5}{2}$ resonance of $\alpha-\mathrm{Al}_{2} \mathrm{O}_{3}$ powder, using various sweep rates. As pointed out in Figure 6.4, signal averaging was sometimes used but the relative intensities of the peaks are normalized to the same number of sweeps. The dc field was about $10 \mathrm{G}$. 

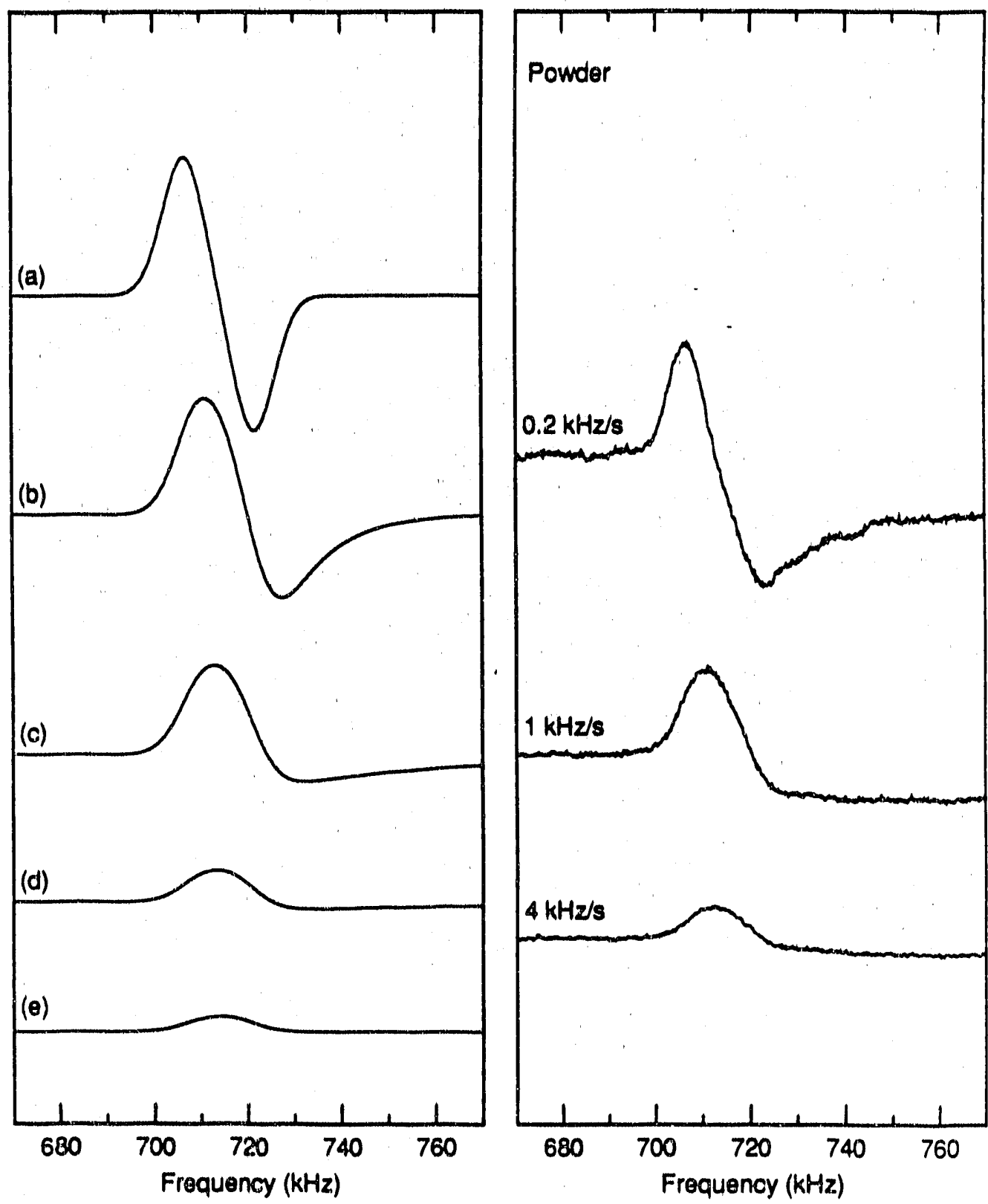

XBL $908-2802$

Figure 6.12: Simulated (left) and experimental forward sweeps of the $\alpha-\mathrm{Al}_{2} \mathrm{O}_{3}$ powder sample, using the $\pm \frac{3}{2} \leftrightarrow \pm \frac{5}{2}$ resonance, for various sweep rates. The applied dc field was about $10 \mathrm{G}$. 
in a powder sample. This is demonstrated in Figure 6.10.

\subsection{Petalite, $\mathrm{LiAlSi}_{4} \mathrm{O}_{10}$}

Petalite $\left(\mathrm{LiAlSi}_{4} \mathrm{O}_{10}\right)$ is a polycrystalline lithium ore with only one distinct ${ }^{27} \mathrm{Al}$ site. Folded $\mathrm{Si}_{2} \mathrm{O}_{5}$ layers are linked by $\mathrm{Li}$ and $\mathrm{Al}$ tetrahedra. The $\mathrm{AlO}_{4}$ tetrahedra are distorted such that the Al-O bond distances are $1.742 \AA$ and $1.732 \AA$ [125]. The two $\mathrm{O}-\mathrm{Al}-\mathrm{O}$ bond angles are $116.97^{\circ}$ and $113.73^{\circ}$. The spectrum of petalite is shown in Figure 6.13, and the two resonances are at $834 \pm 5 \mathrm{kHz}$ and $1314 \pm 5 \mathrm{kHz}$. Using Equations 2.39 and 2.40 we find that $\frac{e^{2} q Q}{h}=4.56 \pm 0.01 \mathrm{MHz}$ and $\eta=0.47 \pm$ 0.1 . This measurement is in agreement with early estimates [126], and was later confirmed by data from dynamic-angle-spinning (DAS) experiments [127]. DAS experiments gave $\frac{\mathrm{e}^{2} q Q}{h}=4.62 \pm 0.05 \mathrm{MHz}$ and $\eta=0.48 \pm 0.03$.

Two items of interest in this petalite spectum are the linewidth of the resonance and signal intensity. The linewidths of the resonances are relatively large and indicate a considerable amount of structural disorder in this natural mineral sample. From structural considerations, dipolar broadening is not expected to contribute significantly to the linewidth. The large linewidth is more likely due to a distribution in electric field gradients. The relatively large quadrupole moment $(e Q)$ of ${ }^{27} \mathrm{Al}$ makes matters worse. This is because a small change in electric field gradients produces a larger change in the distribution of pure NQR resonances. This large quadrupole moment is, however, what allows us to see this resonance. Usually, tetrahedral distortions are not large, but the large electric quadrupole moment of ${ }^{27} \mathrm{Al}$ brings the quadrupole interaction into a frequency range where we can detect it. A perfect tetrahedron has O-Al-O bond angles of $109.5^{\circ}$. 

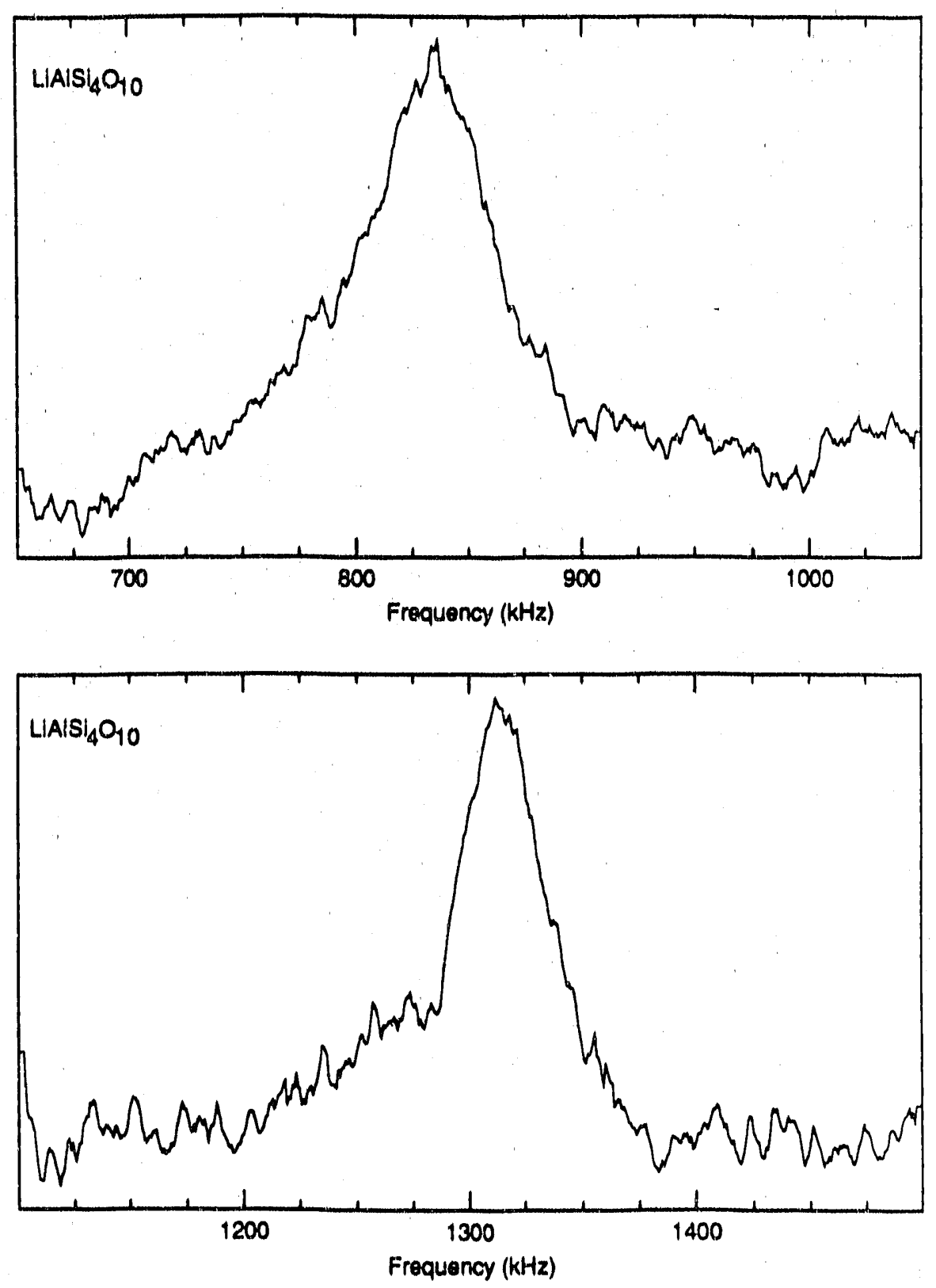

XEL $908-2803$

Figure 6.13: ${ }^{27} \mathrm{Al} \mathrm{NQR}$ powder spectrum of the natural mineral petalite $\left(\mathrm{LiAlSi}_{4} \mathrm{O}_{10}\right)$. The dc field used was about $10 \mathrm{G}$. 
The signal intensity of these two resonances is much smaller than that for $\alpha$ $\mathrm{Al}_{2} \mathrm{O}_{3}$. Several factors may account for this. Firstly, the concentration of ${ }^{27} \mathrm{Al}$ in petalite is smaller than that in $\alpha-\mathrm{Al}_{2} \mathrm{O}_{3}$. Secondly, the signal intensity available in petalite is spread over a much larger frequency range making the signal amplitude look smaller. The final factor is the non-zero asymmetry parameter. As noted in Chapter 2, we expect smaller signals from sites where the asymmetry parameter deviates from zero.

\subsection{Further SQUID NQR Experiments Possible}

In this section, some other further possibilities of the SQUID NQR technique are suggested. An obvious extension of this technique is to apply it to samples containing ${ }^{17} \mathrm{O}$ and other samples containing ${ }^{27} \mathrm{Al}$. Many of the high- $\mathrm{T}_{\mathrm{c}}$ superconductors contain ${ }^{17} \mathrm{O}$ and other quadrupolar nuclei such as ${ }^{63} \mathrm{Cu},{ }^{65} \mathrm{Cu},{ }^{87} \mathrm{Sr},{ }^{135} \mathrm{Ba},{ }^{137} \mathrm{Ba}$, and ${ }^{139} \mathrm{La}$. Taking $\mathrm{La}_{2-x} \mathrm{Sr}_{x} \mathrm{CuO}_{4}$ as an example, $\mathrm{La}_{1.8} \mathrm{Sr}_{0.2} \mathrm{CuO}_{4}$ is a high- $\mathrm{T}_{c}$ superconductor but $\mathrm{La}_{2-x} \mathrm{Sr}_{x} \mathrm{CuO}_{4}$ (where $0 \leq x<0.2$ ) is not, we'll call the latter stoichiometric precursors. Once this technique has been successfully applied to simpler ${ }^{17} \mathrm{O}, \mathrm{Cu},{ }^{87} \mathrm{Sr}$, and ${ }^{139} \mathrm{La}$ compounds, one can study these compounds which are stoichiometric precursors to high- $\mathrm{T}_{c}$ superconductors and possibly shed some light on what gives the high- $\mathrm{T}_{c}$ superconductors their important qualities. If the particle sizes are small enough, one may even be able to study these high- $T_{c}$ superconductors directly. Many technologically important substances, such as zeolites, contain ${ }^{27} \mathrm{Al}$. These zeolites are also excellent substances to study with this SQUID NQR technique.

One may wonder what happens when there is more than one distinct ${ }^{27} \mathrm{Al}$ site 
in a sample. For example, if the sample has two sites then the NQR spectrum will have four resonances, two for each site. The experimentalist has two options in trying to determine which resonance is the counterpart of the other, i.e. which of the other three peaks is from the same site as a given peak. The first, and easiest, option is a process of elimination since the resonance frequencies from a particular site must obey certain mathematical restrictions. These restrictions are $\frac{1}{2} \nu_{Q, H} \leq \nu_{Q, L} \leq \nu_{Q, H}$ for resonances from one particular site. If this process of elimination can not be used, then one has to perform a classic double resonance experiment $[18,32]$. This option consists of irradiating one peak with $\mathrm{rf}$, and taking the spectrum to determine which of the other three peaks is influenced in intensity. The peak whose intensity changes the most is its counterpart. Once the pairing of peaks has been accomplished, the experimentalist can go on to determine the quadrupolar parameters for each site.

Improvements to the spectrometer would be to make the probe variable-temperature. This would enable the study of phase transitions in compounds and allow one to determine how the NQR resonance(s) change as the structure changes. A modification which would make experiments more convenient, would be to enable the experimentalist to change the static magnetic field without warming up the entire probe, as has to be done now. 


\section{Chapter 7}

\section{I = 1 NQR Theory and Results}

\subsection{Introduction}

In the two preceeding chapters it was demonstrated how this SQUID NQR technique may be applied to samples which contain half-odd-integer spin quadrupolar nuclei. The obvious question is whether this technique may be applied to integer spin quadrupolar nuclei. In this chapter, this possibility is addressed and one spin $I=1$ result is presented.

The factor that determines the feasibility of this technique is the ability to generate $M_{z}$ (magnetization along the $z$-axis), the z-axis being defined as the direction of the static magnetic field and the axis of the pickup coil. The eigenvalues and eigenstates of integer spin nuclei behave differently from those of half-odd-integer spin, as was seen in the boron chapter. The following is a description of $\operatorname{spin} I=1$ nuclei, their eigenvalues and eigenstates. The more important nuclei with spin $I=1$ are ${ }^{2} \mathrm{H}$ and ${ }^{14} \mathrm{~N}$, but since ${ }^{2} \mathrm{H}$ rarely has resonances in the operating frequency range of this spectrometer, this discussion is more pertinent to ${ }^{14} \mathrm{~N}$. 


\subsection{Theory for $I=1$ Nuclei}

To facilitate this discussion, we take the example of a spin $I=1$ quadrupolar nucleus in a static magnetic field such that the direction of the weak magnetic field is along the z-axis of the quadrupolar interaction. The energy levels are given $[109,128,129]$ by

$$
\begin{aligned}
& E_{x}=\frac{e^{2} q Q}{4}\left[1+\eta\left(1+z^{2}\right)^{\frac{1}{2}}\right] \\
& E_{y}=\frac{e^{2} q Q}{4}\left[1-\eta\left(1+z^{2}\right)^{\frac{1}{2}}\right] \\
& E_{z}=-\frac{e^{2} q Q}{2}
\end{aligned}
$$

where $z=\frac{\gamma B_{0}}{\left(\frac{a^{2} q q}{d \hbar}\right) \eta}$ and the corresponding eigenstates are

$$
\begin{aligned}
& |x\rangle=\cos \theta|+1\rangle+\sin \theta|-1\rangle \\
& |y\rangle=\sin \theta|+1\rangle-\cos \theta|-1\rangle
\end{aligned}
$$

and

$$
|z\rangle=|0\rangle \text {. }
$$

The trigonometric terms above are defined as $\cos \theta=\frac{1}{\sqrt{2}}\left[1+z^{2}+z\left(1+z^{2}\right)^{\frac{1}{2}}\right]^{-\frac{1}{2}}$ and $\sin \theta=\frac{1}{\sqrt{2}}\left[1+z^{2}-z\left(1+z^{2}\right)^{\frac{1}{2}}\right]^{-\frac{1}{2}}$. The energy levels are shown in Figure 7.1 and the kets $|+1\rangle,|-1\rangle$, and $|0\rangle$ are eigenstates of $I_{z}$.

In the absence of a magnetic field, $|x\rangle$ is an eigenfunction of $I_{x},|y\rangle$ is an eigenfunction of $I_{y}$, and $|z\rangle$ is an eigenfunction of $I_{z}$, and all the corresponding eigenvalues are zero. When a weak, static magnetic field is applied along the z-axis of the quadrupolar interaction, it is found that

$$
\begin{aligned}
& \left\langle I_{x}\right\rangle=\left\langle I_{y}\right\rangle=0 \text { for all eigenstates, } \\
& \left\langle z\left|I_{z}\right| z\right\rangle=0
\end{aligned}
$$




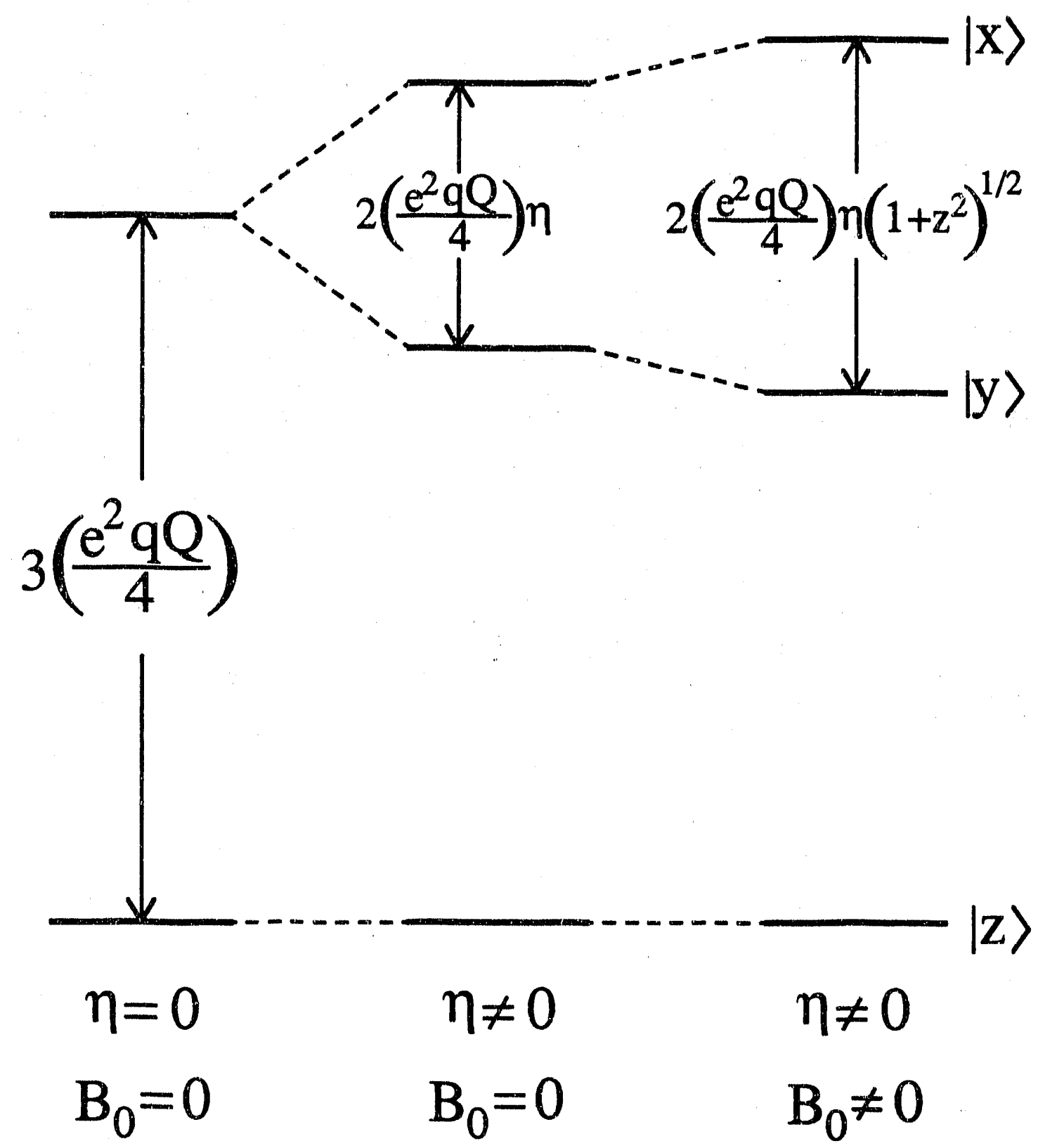

Figure 7.1: The energy levels of a spin $I=1$ nuclei under a variety of conditions. The weak magnetic field is applied along the $z$-axis of the quadrupolar PAS and $z=\frac{\gamma B_{0}}{\left(\frac{e^{2} q Q}{h}\right)_{\eta}}$. Application of the magnetic field moves the energy levels symmetrically, but in opposite directions, about the original energy levels. 
and

$$
\left\langle x\left|I_{z}\right| x\right\rangle=-\left\langle y\left|I_{z}\right| y\right\rangle=-\frac{z}{\left(1+z^{2}\right)^{\frac{1}{2}}} .
$$

Leppelmeier and Hahn have also worked out all of the above when the magnetic field is applied along the $x$ or $y$ directions [109].

Proceeding further, we wish to get an estimate of the magnitude of the signal. At thermal equilibrium, the z-magnetization of the sample is

$$
\begin{array}{r}
M_{z} \propto P_{|z\rangle} I_{z,|z\rangle}+P_{|y\rangle} I_{z,|y\rangle}+P_{|x\rangle} I_{z,|x\rangle}, \\
M_{z} \propto \frac{z}{\left(1+z^{2}\right)^{\frac{1}{2}}}\left(P_{|y\rangle}-P_{|x\rangle}\right),
\end{array}
$$

where $I_{z,|i\rangle}=\left\langle i\left|I_{z}\right| i\right\rangle$. As we pass through and irradiate one NQR transition (e.g. $|z\rangle \leftrightarrow|y\rangle)$, the induced z-magnetization is given by

$$
\begin{array}{r}
M_{z}^{\prime} \propto P_{|z\rangle}^{\prime} I_{z,|z\rangle}+P_{|y\rangle}^{\prime} I_{z,|y\rangle}+P_{|x\rangle} I_{z,|x\rangle}, \\
M_{z}^{\prime} \propto \frac{z}{\left(1+z^{2}\right)^{\frac{1}{2}}}\left(P_{|y\rangle}^{\prime}-P_{|x\rangle}\right) .
\end{array}
$$

Thus, the change in the z-magnetization of the sample is

$$
\begin{aligned}
\Delta M_{z} & =M_{z}^{\prime}-M_{z} \\
& \propto \frac{z}{\left(1+z^{2}\right)^{\frac{1}{2}}}\left[\left(P_{|y\rangle}^{\prime}-P_{|x\rangle}\right)-\left(P_{|y\rangle}-P_{|x\rangle}\right)\right] \\
& \propto \frac{z}{\left(1+z^{2}\right)^{\frac{1}{2}}}\left[P_{|y\rangle}^{\prime}-P_{|y\rangle}\right] .
\end{aligned}
$$

Generating a large change in the population of the $|y\rangle$ state, $\left(P_{|y\rangle}^{\prime}-P_{|y\rangle}\right)$, is usually not a problem. The factor which really affects the change in z-magnetization, and thus the size of the signal, is the $\frac{z}{\left(1+z^{2}\right)^{\frac{1}{2}}}$ term, $F(z)$.

A plot of $F(z)$ versus $z$ is given in Figure 7.2. This figure tells us that large signals are possible only when $z$ is relatively large. Since $z=\frac{\gamma B_{0}}{\left(\frac{c^{2} q Q}{4 h}\right)_{\eta}}$, we may 
$F(z)$ versus $z$

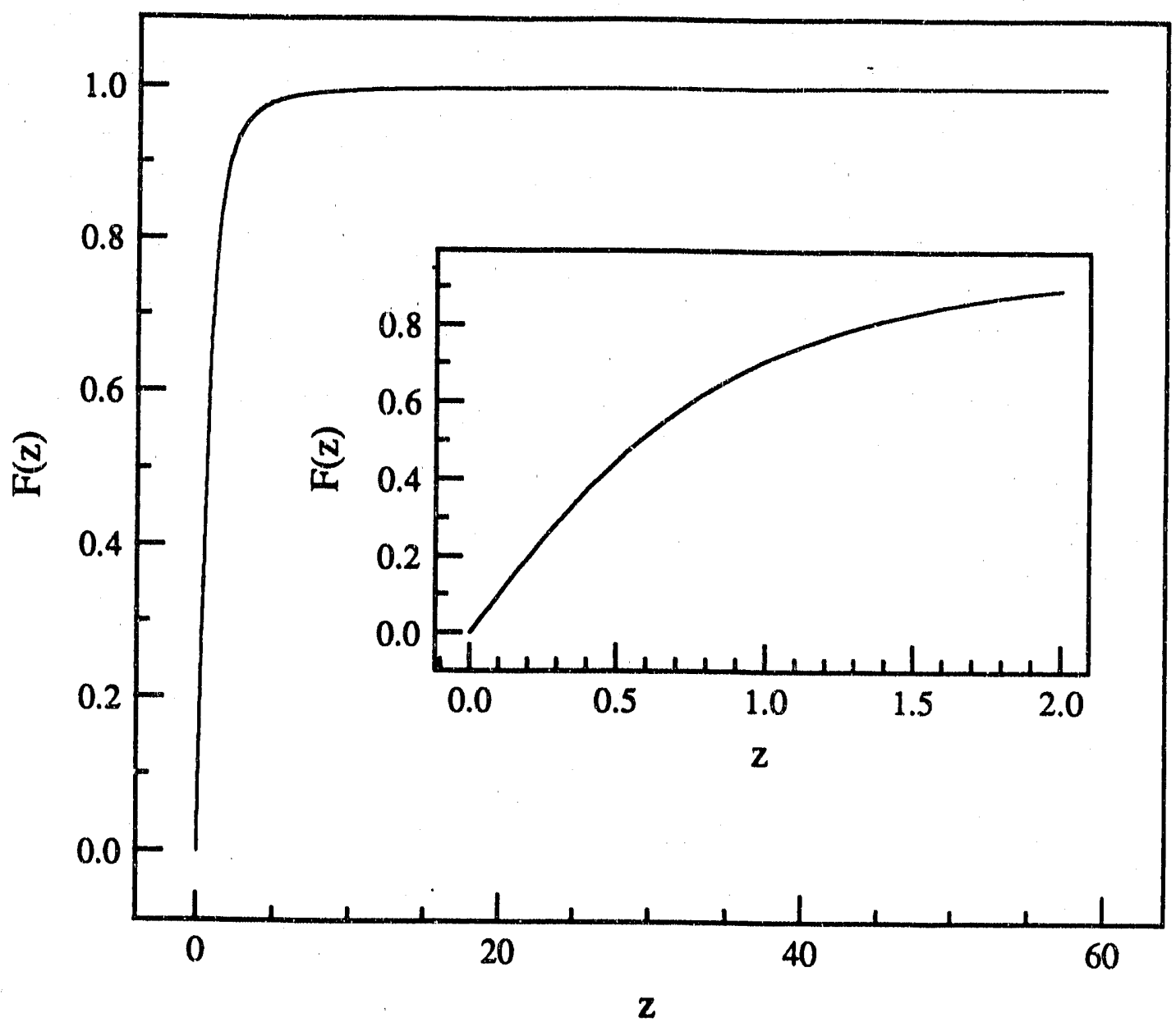

Figure 7.2: Signal intensity versus $z$ factor where $z=\frac{\gamma B_{0}}{\left(\frac{e^{2} g Q}{4 h}\right) \eta}$ and $F(z)=$ $\frac{z}{\left(1+z^{2}\right)^{\frac{1}{2}}} \propto M_{z}$, the z-magnetization of the sample. The inset provides a better view of $F(z)$ for low values of $z$. 
be tempted to maximize $B_{0}$ and even then, large signals are possible only when the product of $\frac{e^{2} q Q}{h}$ and $\eta$ is small. Maximizing $B_{0}$ is not a good idea because the signal available will be spread out over a large frequency range and make the signal intensity smaller. This tells us that reasonable signal intensity is possible only when $\frac{e^{2} q Q}{h}$ is small and $\eta$ is close to zero. Figure 7.3 is a plot of $F(z)$ versus $\eta$ while setting $\gamma B_{0}=11 \mathrm{kHz}$ and $\frac{e^{2} g Q}{h}=5000 \mathrm{kHz}$.

\subsection{Solid Nitrogen, $\alpha-\mathrm{N}_{2}$}

We obtained the SQUID NQR spectrum of solid $\alpha-\mathrm{N}_{2}$ (natural abundance in ${ }^{14} \mathrm{~N}$ ) as shown in Figure 7.4. The magnetic field used was $35 \mathrm{G}$ and the pure NQR resonance frequency was determined to be $3488 \pm 2 \mathrm{kHz}$. The high spin density, short spin-lattice relaxation time, known quadrupolar interaction strength, and very small asymmetry parameter made this sample an excellent choice. The resonance frequency was in excellent agreement with previous measurements of $3487.73 \pm$ $0.03 \mathrm{kHz}$ at $4.2 \mathrm{~K}[130,131]$. Scott and coworkers were able to place a limit on $\eta$ at $\eta \leq 0.00016$. From an analysis of the linewidth of our resonance at $35 \mathrm{G}$, we can place a limit of $\eta \leq 0.006$. We did not try to place a better limit on $\eta$ by reducing the field strength since the signal intensity would also decrease. The signal strength of the $\alpha-\mathrm{N}_{2}$ sample was very low, requiring a total of 20 forward and reverse sweeps to obtain the spectrum shown. Before proceeding further, a numerical estimate of certain parameters are of interest. If one takes $\nu_{Q}=3488 \mathrm{kHz}$ and $\eta=0$, then the quadrupole coupling constant is $\frac{e^{2} q Q}{h}=4651 \mathrm{kHz}$. Using this value of the quadrupole coupling constant along with $\eta=0.00016$ and $B_{0}=35$ $\mathrm{G}$, then $z \doteq 58$. This shows that even when $z$ is large, signal intensity is low. So 
$\mathrm{F}(\mathrm{z})$ versus $\eta$

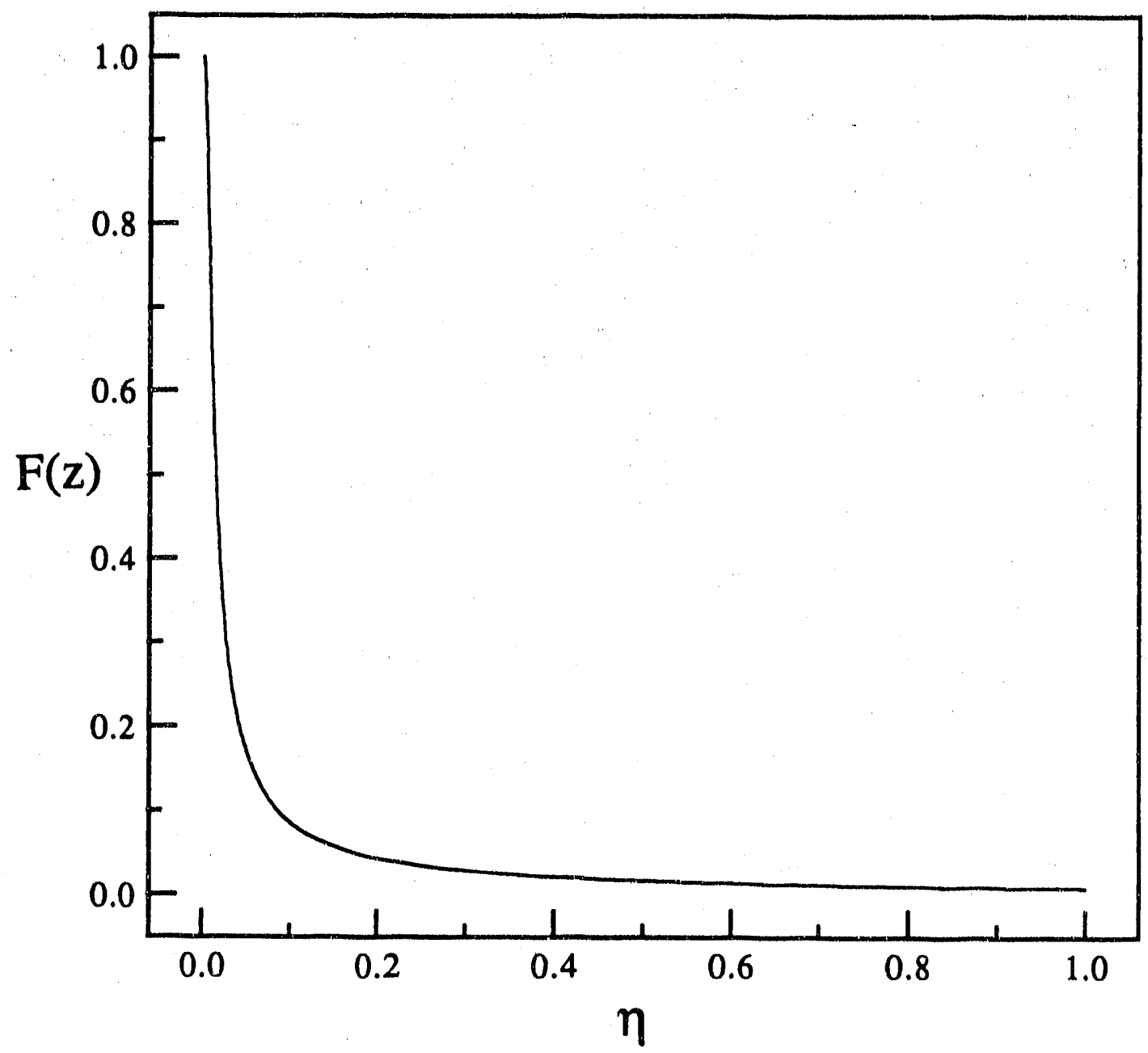

Figure 7.3: Signal intensity versus $\eta$ using $\gamma B_{0}=11 \mathrm{kHz}$ and $\frac{e^{2} q Q}{h}=5000 \mathrm{kHz}$. 


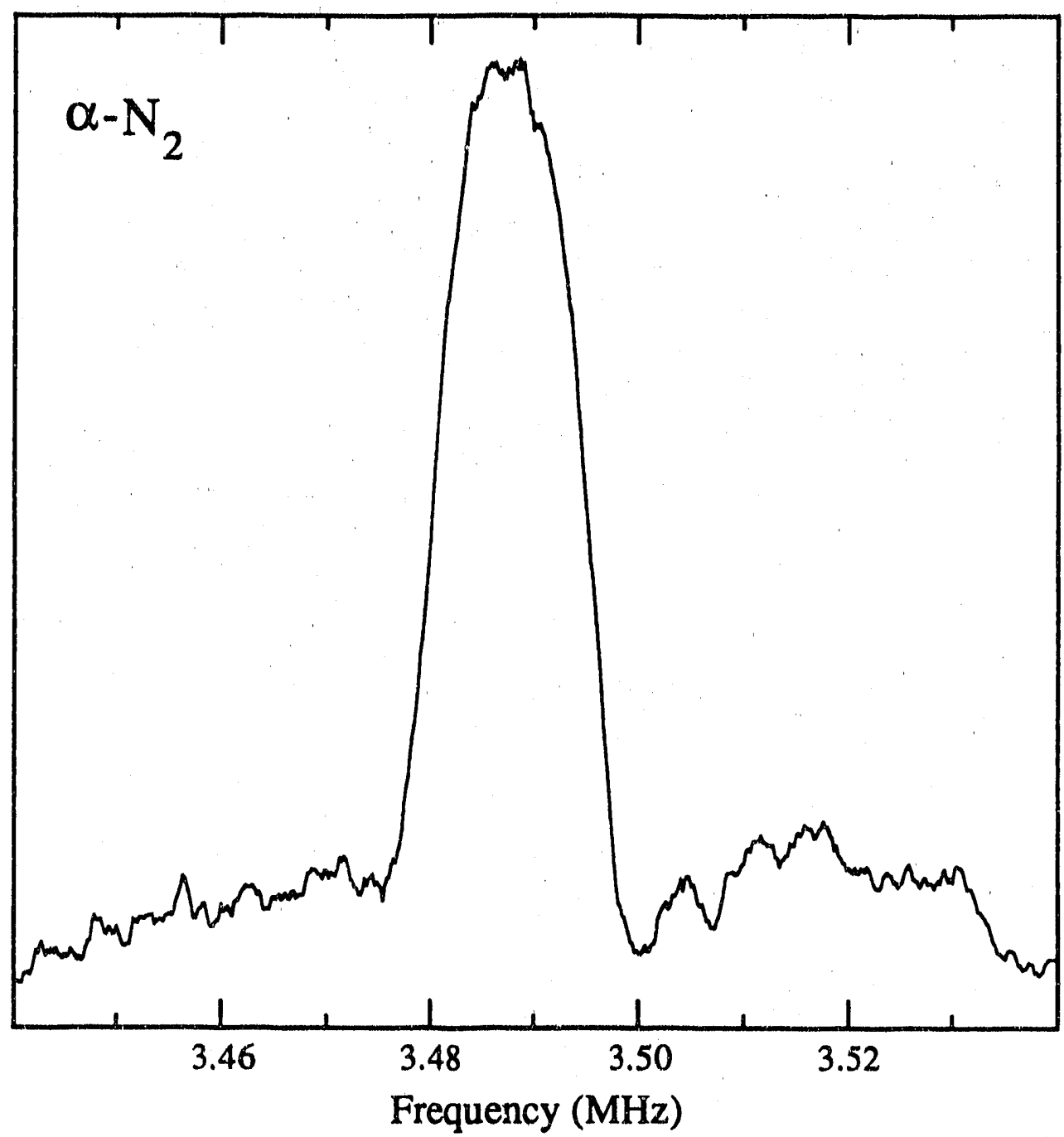

Figure 7.4: Spectrum of $\alpha-\mathrm{N}_{2}$. A total of 20 forward and reverse sweeps were required to obtain this spectrum. A recycle delay of $250 \mathrm{~s}$ was implemented between sweeps. The very low asymmetry parameter of $\alpha-\mathrm{N}_{2}$ allows for reasonable signal intensity. 
samples where $\eta$ is not close to zero will, according to Figure 7.3, give much smaller signal intensities.

The low signal intensity for ${ }^{14} \mathrm{~N}$ samples is not a great loss since resonances that appear in this region of a few megahertz and higher are not difficult to detect by conventional pure NQR. If the signal-to-noise ratio of this technique is increased, it may become possible to investigate $\mathrm{N}_{2}$ molecules in confined spaces, such as in the cages of zeolites. One could try to determine how the size and the loading of the cages affects the asymmetry parameter $(\eta)$ of the quadrupolar interaction.

In summary, this SQUID NQR technique has rather limited application to the study of samples containing integer spin quadrupolar nuclei. On the other hand, it is a viable technique by which to study samples containing half-odd-integer spin quadrupolar nuclei where the quadrupolar interactions fall into the intermediate strength regime. 


\section{Appendix A}

\section{Analytical Solution to the}

\section{Zero-Field Energy Levels of a}

\section{Spin $I=\frac{\mathbf{5}}{\mathbf{2}}$ Nucleus}

This appendix discusses the energy levels of a spin $I=\frac{5}{2}$ nucleus, involved in a quadrupolar interaction, in zero magnetic field. Most approaches to this problem use perturbation theory or purely numerical solutions to a cubic secular equation [5, $132,133,134,135,136]$. An analytical solution is presented here. The quadrupolar Hamiltonian may be written alternatively [41] as

$$
H_{Q}=\frac{e^{2} q Q}{4 I(2 I-1)}\left[3 I_{z}^{2}-I^{2}+\frac{\eta}{2}\left(I_{+}^{2}+I_{-}^{2}\right)\right] \text {. }
$$

An especially convenient matrix representation of the Hamiltonian [137] is 


$$
\begin{aligned}
& \left|+\frac{5}{2}\right\rangle \quad\left|+\frac{1}{2}\right\rangle \quad\left|-\frac{3}{2}\right\rangle \quad\left|+\frac{3}{2}\right\rangle \quad\left|-\frac{1}{2}\right\rangle \quad\left|-\frac{5}{2}\right\rangle \\
& \left\langle+\frac{5}{2}\right|\left(\begin{array}{cccccc}
\frac{A}{4} & \frac{A \eta}{4 \sqrt{10}} & 0 & 0 & 0 & 0
\end{array}\right. \\
& \left\langle+\frac{1}{2}|| \begin{array}{llllll}
\frac{A \eta}{4 \sqrt{10}} & -\frac{A}{B} & \frac{3 A \eta}{20 \sqrt{2}} & 0 & 0 & 0
\end{array}\right. \\
& \left\langle-\frac{3}{2}\right| \quad 0 \quad \frac{3 A \eta}{20 \sqrt{2}} \quad-\frac{A}{20} \quad 0 \quad 000 \\
& \left\langle+\frac{3}{2}\right| \quad 0 \quad 000 \begin{array}{llll}
\frac{A}{20} & \frac{3 A \eta}{20 \sqrt{2}} & 0
\end{array} \\
& \left\langle-\frac{1}{2}|| \begin{array}{llllll}
0 & 0 & 0 & \frac{3 A \eta}{20 \sqrt{2}} & -\frac{A}{5} & \frac{A \eta}{4 \sqrt{10}}
\end{array}\right. \\
& \left\langle-\frac{5}{2}\right|\left(\begin{array}{llllll}
0 & 0 & 0 & 0 & \frac{A \eta}{4 \sqrt{10}} & \cdots
\end{array}\right)
\end{aligned}
$$

where $A=e^{2} q Q$.

It is of importunce to note that the $\left| \pm m^{\prime}\right\rangle$ states remain degenerate for all value of $\eta$ in the absence of a magnetic field. This is due to the time-reversal symmetry which is posscssed by the half-odd-integer quadrupolar nuclei $[41,138]$. This allows us to reduce the problem of having to deal with a $6 \times 6$ matrix to only a $3 \times 3$ matrix. To find the eigenvalues of this $3 \times 3$ matrix we need to solve the equation

$$
\left|\begin{array}{ccc}
\frac{A}{4}-\lambda & \frac{A \eta}{4 \sqrt{10}} & 0 \\
\frac{A \eta}{4 \sqrt{10}} & -\frac{A}{5}-\lambda & \frac{3 A \eta}{20 \sqrt{2}} \\
0 & \frac{3 A \eta}{20 \sqrt{2}} & -\frac{A}{20}-\lambda
\end{array}\right|=0
$$

and this yields the cubic secular equation

$$
\lambda^{3}+\left(\frac{-21 A^{2}}{400}-\frac{7 \eta^{2} A^{2}}{400}\right) \lambda+\left(\frac{\eta^{2} A^{3}}{400}-\frac{A^{3}}{400}\right)=0 .
$$

Following the solution to a cubic equation [139], except the symbols $A$ and $B$ in reference [139] have been changed to $C$ and $D$ respectively, one gets

$$
C=\frac{A}{20}\left[-10\left(\eta^{2}-1\right)+10 Z\right]^{\frac{1}{3}}
$$




$$
D=\frac{A}{20}\left[-10\left(\eta^{2}-1\right)-10 Z\right]^{\frac{1}{3}}
$$

where

$$
10 Z=\left[-\frac{343}{27} \eta^{6}-\frac{43}{3} \eta^{4}-543 \eta^{2}-243\right]^{\frac{1}{2}}
$$

$C$ and $D$ are complex conjugates and can be rewritten as

$$
\begin{aligned}
& C=\frac{A}{20}\left[-10\left(\eta^{2}-1\right)+i P\right]^{\frac{1}{3}}, \\
& D=\frac{A}{20}\left[-10\left(\eta^{2}-1\right)-i P\right]^{\frac{1}{3}},
\end{aligned}
$$

where

$$
P=\left[\frac{343}{27} \eta^{6}+\frac{43}{3} \eta^{4}+543 \eta^{2}+243\right]^{\frac{1}{2}}
$$

and $P$ is always real and positive. The determination of the cube roots of these complex numbers may be facilitated by rewriting them in polar or trigonometric form [140]. Expressing $C$ and $D$ in polar form one gets

$$
\begin{aligned}
& C=\frac{A}{20} r^{\frac{1}{3}} e^{i\left(\frac{\theta}{3}\right)}, \\
& D=\frac{A}{20} r^{\frac{1}{3}} e^{-i\left(\frac{\theta}{3}\right)}
\end{aligned}
$$

where

$$
\begin{aligned}
& r=\left\{\left[-10\left(\eta^{2}-1\right)\right]^{2}+P^{2}\right\}^{\frac{1}{2}} \\
& P=\left[\frac{343}{27} \eta^{6}+\frac{43}{3} \eta^{4}+543 \eta^{2}+243\right]^{\frac{1}{2}}
\end{aligned}
$$

and

$$
\theta=\arctan \left[\frac{-P}{10\left(\eta^{2}-1\right)}\right]
$$


Finally, one gets the following eigenvalues

$$
\begin{aligned}
& E_{1}=\frac{-A}{20} r^{\frac{1}{3}}\left[-2 \cos \left(\frac{\theta}{3}\right)\right], \\
& E_{2}=\frac{-A}{20} r^{\frac{1}{3}}\left[\cos \left(\frac{\theta}{3}\right)+\sqrt{3} \sin \left(\frac{\theta}{3}\right)\right],
\end{aligned}
$$

and

$$
E_{3}=\frac{-A}{20} r^{\frac{1}{3}}\left[\cos \left(\frac{\theta}{3}\right)-\sqrt{3} \sin \left(\frac{\theta}{3}\right)\right]
$$

where

$$
\begin{aligned}
A & =e^{2} q Q \\
r^{\frac{1}{3}} & =\left(\frac{7}{3} \eta^{2}+7\right)^{\frac{1}{2}} \\
P & =\left[\frac{343}{27} \eta^{6}+\frac{43}{3} \eta^{4}+543 \eta^{2}+243\right]^{\frac{1}{2}}
\end{aligned}
$$

and

$$
\theta=\arctan \left[\frac{-P}{10\left(\eta^{2}-1\right)}\right]
$$

The two resonance frequencies are

$$
\begin{aligned}
& \nu_{Q, L}=\left|E_{2}-E_{3}\right|=\frac{A}{20 h} r^{\frac{1}{3}}(2 \sqrt{3}) \sin \left(\frac{\theta}{3}\right), \\
& \nu_{Q, H}=\left|E_{1}-E_{3}\right|=\frac{A}{20 h} r^{\frac{1}{3}}\left[3 \cos \left(\frac{\theta}{3}\right)-\sqrt{3} \sin \left(\frac{\theta}{3}\right)\right] .
\end{aligned}
$$

With the above equations, one can calculate where to expect the two pure NQR resonances when $\frac{e^{2} q Q}{h}$ and $\eta$ are known.

It is also very useful to be able to calculate $\frac{e^{2} q Q}{h}$ and $\eta$ from the frequencies of the two pure quadrupole resonances. This can be done by taking the ratio of Equations A.19 and A.18 to get

$$
\frac{\nu_{Q, H}}{\nu_{Q, L}}=\frac{\sqrt{3}}{2} \cot \left(\frac{\theta}{3}\right)-\frac{1}{2}
$$


or

$$
\theta=3 \arctan \left(\frac{\sqrt{3} \nu_{Q, L}}{2 \nu_{Q, H}+\nu_{Q, L}}\right)
$$

This gives the value of $\theta$ which corresponds to the two known resonance frequencies. Equations A.16 and A.17 tell us that $\theta$ is a function of $\eta$ only, and varies monotonically from $57.31982^{\circ}$ to $90^{\circ}$ when $\eta=0$ and $\eta=1$ respectively. This allows one to perform a binary search for the value of $\eta$ which, when input into Equations A.16 and A.17, gives the proper value of $\theta$. Once the correct value of $\eta$ has been determined, Equation A.18 or A.19 can be used to calculate $\frac{e^{2} q Q}{h}$. On the following pages are two programs. The first program calculates the energy levels and resonance frequencies for a spin $I=\frac{5}{2}$ nucleus, in zero fleld, from the quadrupole coupling constant and the asymmetry parameter. The second program will calculate the quadrupole coupling constant and asymmetry parameter from the two resonance frequencies of a spin $I=\frac{5}{2}$ nucleus in zero magnetic field. 
Program Energy;

$\{$ This program gives the energy levels and the resonance frequencies for spin $I=5 / 2$ in zero field.

Var
$P, A, r$, eta, theta
: Real ;
phi, factor, safety
: Real ;
$\mathrm{E} 1, \mathrm{E} 2, \mathrm{E} 3$
: Real ;
lowfreq, hifreq
: Real ;
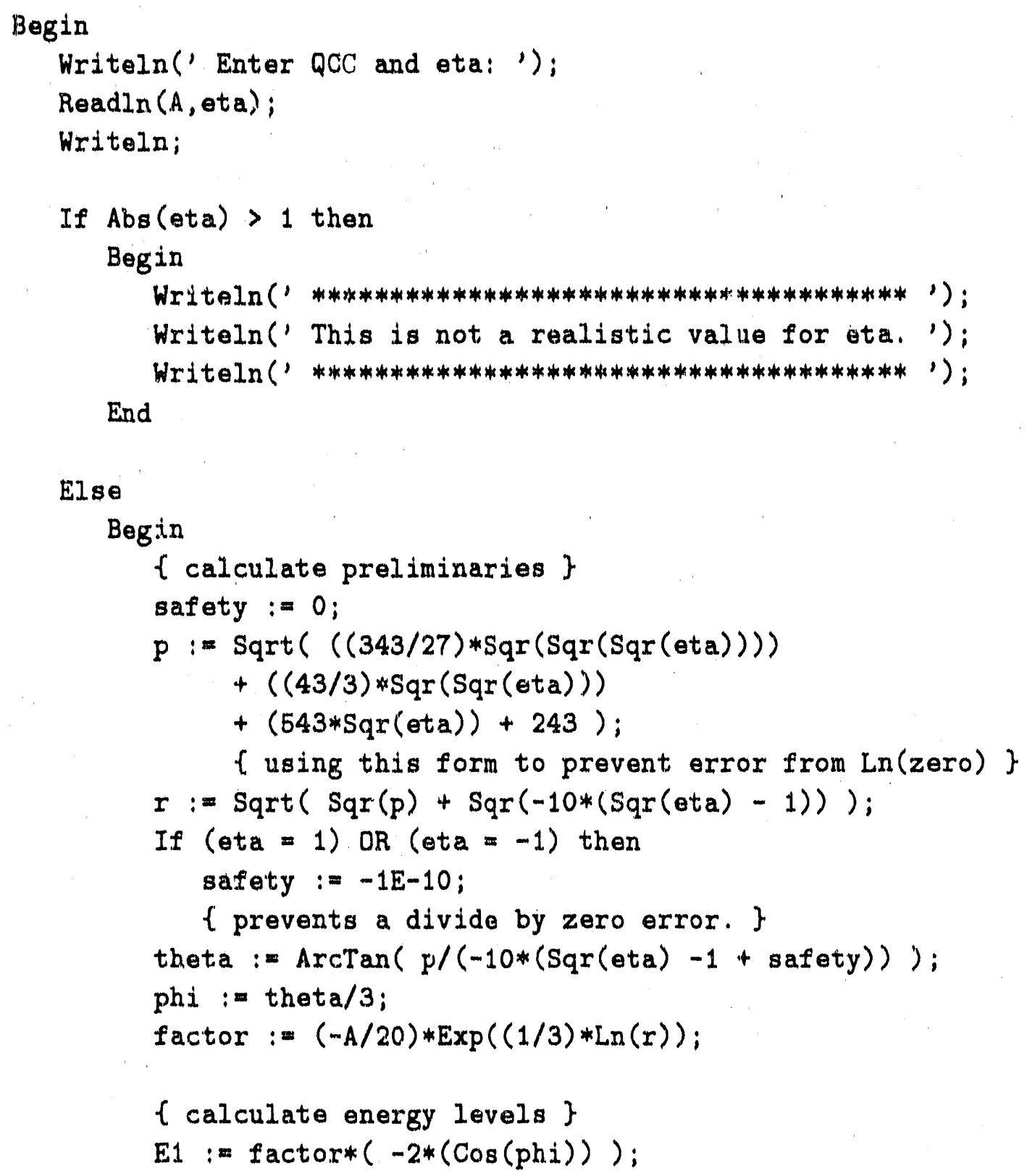


$$
\begin{aligned}
& \text { E2 }:=\text { factor* }(\operatorname{Cos}(p h i)+\operatorname{Sqrt}(3) * \operatorname{Sin}(p h i)) ; \\
& \text { E3 :=factor*( } \operatorname{Cos}(p h i)-\operatorname{Sqrt}(3) * \operatorname{Sin}(p h i)) ;
\end{aligned}
$$

$\{$ calculate resonance frequencies \}

lowfreq : $=\mathrm{AbB}(\mathrm{E2}-\mathrm{E} 3)$;

hifreq $:=\operatorname{Abs}(E 1-E 3)$;

Writeln('The low frequency resonance is at:', lowfreq); Writeln('The high frequency resonance is at:', hifreq); End;

End. \{ Program Energy $\}$ 
Program Frequency;

$\{$ This is program gives the QCC and eta for the two resonances input for a spin $I=5 / 2$ spin system in zero field. $\}$

$\operatorname{Var}$

lowfreq, hifreq, tempfreq, theta

Ssquared, eta, etaincrement

Frac, numerator, denominator

ratiolimit, safety 1, safety 2

p,r.A

n

: Real ;

: Real ;

: Real ;

: Real ;

: Real ;

: Integer ;

Begin

Writeln(' Enter the low and high frequency resonances: ');

Readln (lowfreq, hifreq);

lowfreq := Abs(lowfreq);

hifreq := Abs (hifreq);

\{ get resonance frequencies in proper order \}

If lowfreq $>$ hifreq Then

begin

tempfreq := lowfreq;

lowfreq := hifreq;

hifreq := tempfreq;

end ;

If ((hifreq/lowf req) $>=1$ ) AND ((hifreq/lowfreq) $<=2$ ) Then begin

theta $:=3 * \operatorname{ArcTan}($ Sqrt $(3) *$ lowfreq $) /((2 *$ hitreq $)$

safety $1:=1 \mathrm{E}-11$;

+ lowfreq));

Ssquared $:=\operatorname{Sqr}(\operatorname{Sin}($ theta $) /(\operatorname{Cos}($ theta $)+\operatorname{safety} 1))$;

$\{$ Ssquared is determined by the resonance frequencies $\}$

eta $:=0.5$;

etaincrement :=0.5;

If $((($ hifreq/Iowf req $)-1)<=1 E-03)$ Then ratiolimit $:=1 \mathrm{E}-06$

$\{$ while criteria less stringent $\}$

Else 


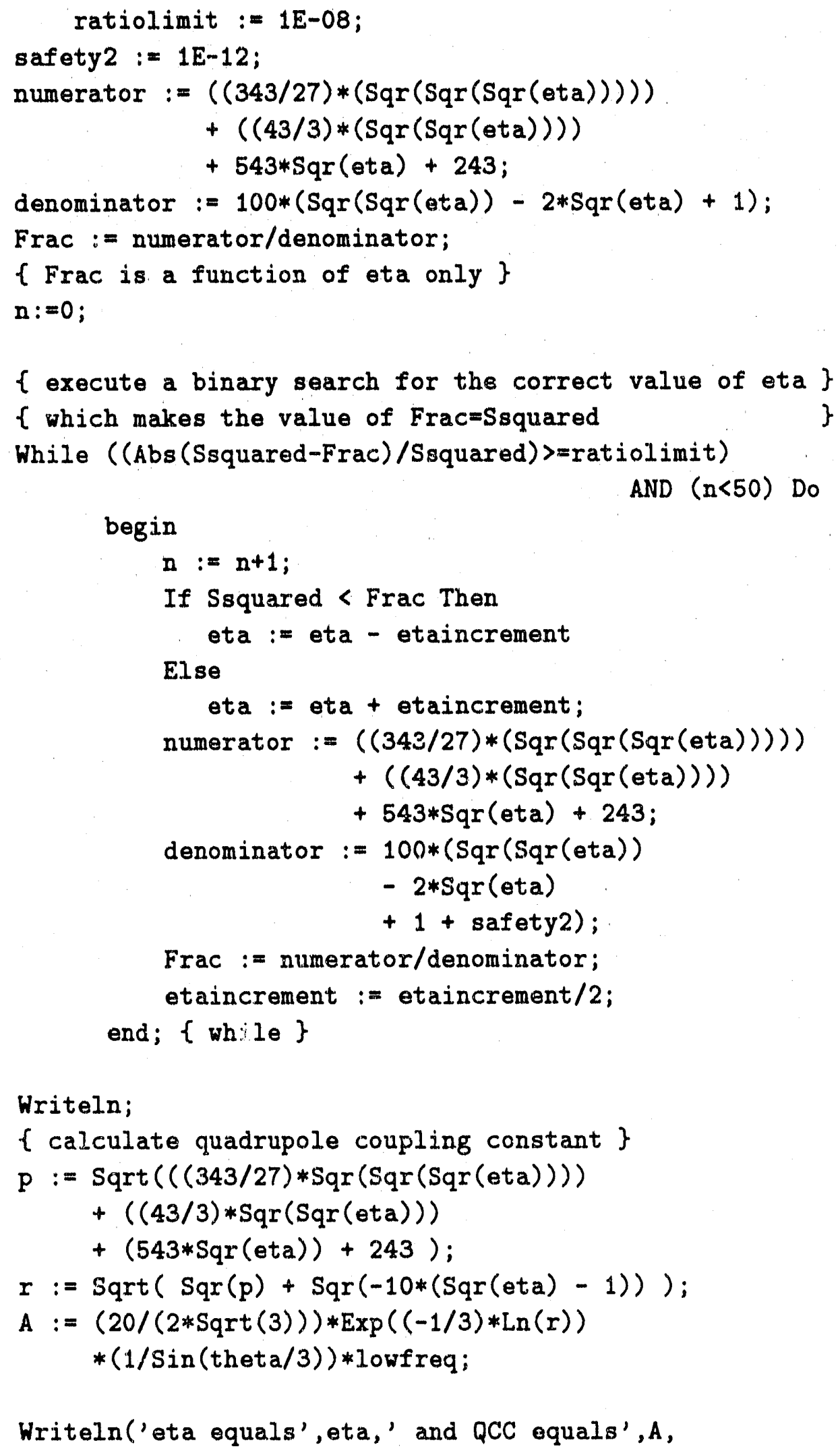




\section{'after ', $n$, ' iterations');}

end $\{$ when ratio of frequencies is in proper range\}

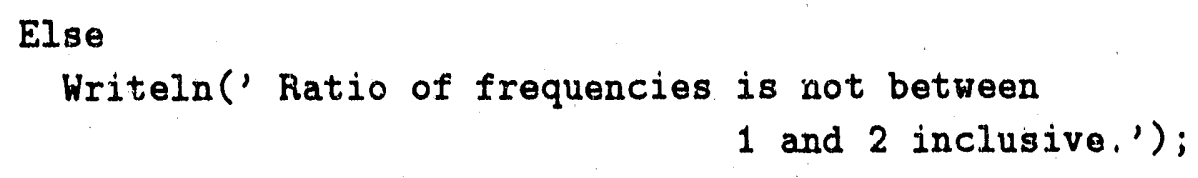

End. \{ Program Frequency \} 


\section{Bibliography}

[1] R. V. Pound, Phys. Rev. 79, 685 (1950).

[2] C. H. Townes and B. P. Dailey, J. Chem. Phys. 17, 782 (1949).

[3] C. H. Townes and B. P. Dailey, J. Chem. Phys. 20, 35 (1952).

[4] C. H. Townes and B. P. Dailey, J. Chem. Phys. 23, 118 (1955).

[5] T. P. Das and E. L. Hahn, Solid State Physics, Supplement 1, edited by F. Seitz and D. Turnbull (Academic Press, New York, 1958).

[6] D. I. Hoult and R. E. Richards, J. Magn. Reson. 24, 71 (1976).

[7] P. Lorrain and D. R. Corson, Electromagnetic Fields and Waves, 2nd edition (W.H. Freeman and Co., San Francisco, 1962).

[8] S. J. Gravina and P. J. Bray, J. Magn. Reson. 89, 515 (1990).

[9] M. H. Cohen and F. Reif, in Solid State Physics, Vol. 5, edited by F. Seitz and D. Turnbull (Academic Press, New York, 1957).

[10] C. P. Slichter, Principles of Magnetic Resonance, 3rd edition (Springer-Verlag, New York, 1989).

[11] G. E. Pake, J. Chem. Phys. 16, 327 (1948).

[12] N. Bloembergen and J. A. Rowland, Acta Metallurg. 1, 731 (1953).

[13] A. Samoson, E. Kundla, and E. Lippmaa, J. Magn. Reson. 49, 350 (1982).

[14] K. T. Mueller, B. Q. Sun, G. C. Chingas, J. W. Zwanziger, T. Terao, and A. Pines, J. Magn. Reson. 86, 470 (1990).

[15] Y. Wu, B. Q. Sun, A. Pines, A. Samoson, and E. Lippmaa, J. Magn. Reson. 89, 297 (1990).

[16] M. Mehring, Principles of High Resolution NMR in Solids, 2nd edition (Springer-Verlag, Berlin, 1983). 
[17] D. B. Zax, A. Bielecki, K. W. Zilm, A. Pines, and D. P. Weitekamp, J. Chem. Phys. 83, 4877 (1985).

[18] D. T. Edmonds, Int. Rev. Phys. Chem. 2, 103 (1982).

[19] J. Clarke, Phys. Today 39, 36 (1986).

[20] J. Clarke, in Superconductor Applications: SQUIDs and Machines, edited by B.B. Schwartz and S. Foner (Plenum Press, New York, 1977).

[21] R. L. Fagaly, Sci. Prog., Oxf. 71, 181 (1987).

[22] J. Knuutila, A. Ahonen, and C. Tesche, J. Low Temp. Phys. 68, 269 (1987).

[23] C. Dean, Phys. Rev. 96, 1053 (1954).

[24] E. P. Day, Phys. Rev. Lett. 29, 540 (1972).

[25] T. Jach, Appl. Phys, Lett. 28, 49 (1976).

[26] A. Abragam and K. Kambe, Phys. Rev. 91, 894 (1953).

[27] P. J. Bray, J. Non-Cryst. Solids 73, 19 (1985).

[28] P. J. Bray, J. Non-Cryst. Solids 75, 29 (1985).

[29] N. Bloembergen, S. Shapiro, P. S. Pershan, and J. O. Artman, Phys. Rev. 114, 445 (1959).

[30] D. Suter and R. R. Ernst, Phys. Rev. B 32, 5608 (1985).

[31] J. I. Steinfeld, Molecules and Radiation (MIT Press, Cambridge, 1981).

[32] D. T. Edmonds, Phys. Rep. 29, 233 (1977).

[33] A. Pines, M. G. Gibby, and J. S. Waugh, J. Chem. Phys. 56, 1776 (1972).

[34] A. Pines, M. G. Gibby, and J. S. Waugh, J. Chem. Phys. 59, 569 (1973).

[35] M. Bloom, E. L. Hahn, and B. Herzog, Phys. Rev. 97, 1699 (1955).

[36] M. J. Weber and E. L. Hahn, Phys. Rev. 120, 365 (1960).

[37] H. E. Petch and K. S. Pennington, J. Chem. Phys. 36, 1216 (1962).

[38] S. Hafner and M. Raymond, Am. Mineral. 52, 1632 (1967).

[39] T. Jach. Observation of Nuclear Quadrupole Resonance with Superconducting Magnetometers. PhD thesis, Stanford University, 1975.

[40] E. P. Wigner, Group Theory and Its Application to the Quantum Mechanics of Atomic Spectra (Academic Press, New York, 1959). 
[41] A. Abragam, Principles of Nuclear Magnetism (Oxford University Press, Oxford, 1961).

[42] J. J. Sakurai, Modern Quantum Mechanics (Benjamin/Cummings Publishing, Menlo Park, 1985).

[43] E. Merzbacher, Quantum Mechanics (John Wiley and Sons, New York, 1970).

[44] J. Clarke, Am. J. Phys. 38, 1071 (1970).

[45] J. Clarke, Proc. IEEE 61, 8 (1973).

[46] F. Wellstood, C. Heiden, and J. Clarke, Rev. Sci. Instrum. 55, 952 (1984).

[47] J. Clarke, W. M. Goubau, and M. B. Ketchen; J. Low Temp. Phys. 25, 99 (1976).

[48] J. Bardeen, L. N. Cooper, and J. R. Schrieffer, Phys. Rev. 106, 162 (1957).

[49] J. Bardeen, L. N. Cooper, and J. R. Schrieffer, Phys. Rev. 108, 1175 (1957).

[50] C. Kittel, Introduction to Solid State Physics (John Wiley and Sons, New York, 1986).

[51] B. D. Josephson, Rev. Mod. Phys, 36, 216 (1964).

[52] W. H. Parker, D. N. Langenberg, A. Denenstein, and B. N. Taylor, Phys. Rev. 177, 639 (1969).

[53] A. Th. A. M. De Waele and R. De Bruyn Ouboter, Physica 41, 225 (1969).

[54] D. E. McCumber, J. Appl. Phys. 39, 2503 (1968).

[55] R. P. Feynman, R. B. Leighton, and M. Sands, The Feynman Lectures on Physics, Vol. 3 (Addison-Wesley Publishing, Reading, 1965).

[56] R. C. Jaklevic, J. Lambe, J. E. Mercereau, and A. H. Silver, Phys. Rev. A 140, 1628 (1965).

[57] M. Tinkham, Introduction to Superconductivity (McGraw-Hill, New York, 1975).

[58] Y. Aharanov and D. Bohm, Phys. Rev. 115, 485 (1959).

[59] R. G. Chambers, Phys. Rev. Lett. 5 , 3 (1960).

[60] J. Clarke, IEEE Trans. Magn. MAG-19, 288 (1983).

[61.] T. Sleator, E. L. Hahn, M. B. Heaney, C. Hilbert, and J. Clarke, Phys. Rev. Lett. 57, 2756 (1986).

[62] T. Sleator, E. L. Hahn, C. Hilbert, and J. Clarke, Phys. Rev. Lett. 55, 1742 (1985). 
[63] C. Hilbert, J. Clarke, T. Sleator, and E. L. Hahn, Appl. Phys. Lett. 47, 637 (1985).

[64] T. Sleator, E. L. Hahn, C. Hilbert, and J. Clarke, Phys. Rev, B 36, 1969 (1987).

[65] J. H. Claassen, J. Appl. Phys, 46, 2268 (1975).

[66] J. Knuutila, S. Ahlfors, A. Ahonen, J. Hallstrom, M. Kajola, O. V. Lounasmaa, V. Vilkman, and C. Tesche, Rev. Sci. Instrum. 58, 2145 (1987).

[67] R. P. Giffard, R. A. Webb, and J. C. Wheatley, J. Low Temp. Phys. 6, 533 (1972).

[68] P. Horowitz and W. Hill, The Art of Electrunics (Cambridge University Press, Cambridge, 1980).

[69] C. D. Motchenbacher and F. C. Fitchen, Low-Noise Electronic Design (John Wiley and Sons, New York, 1973).

[70] R. N. Bracewell, The Fourier Transform and Its Applications, 2nd edition (McGraw-Hill, New York, 1986).

[71] S. E. Nave and P. G. Huray, Rev. Sci. Instrum. 51, 591 (1980).

[72] L. J. Friedman, A. K. M. Wennberg, S. N. Ytterboe, and H. M. Bozler, Rev. Sci. Instrum. 57, 410 (1986).

[73] R. V. Chamberlain, L. A. Moberly, and O. G. Symko, J. Low Temp. Phys. 35, 337 (1979).

[74] M. Kohl, M. Odehnal, V. Petričcek, and R. Tichý, in SQUID '85Superconducting Quantum Interference Devices and their Applications, edited by H.D. Hahlbohm and H. Lübbig (Walter de Gruyter, Berlin, 1985).

[75] B. S. Deaver, Jr., T. J. Bucelot, and J. J. Finley, in Future Trends in Superconductive Electronics, edited by B.S. Deaver, Jr., C.M. Falco, J.H. Harris, and S.A. Wolf (American Institute of Physics, New York, 1978).

[76] J. S. Philo and W. M. Fairbank, Rev. Sci. Instrum. 48, 1529 (1977).

[77] M. Cerdono, F. Mogno, G. L. Romani, C. Messana, and C. Gramaccioni, Rev. Sci. Instrum. 48, 300 (1977).

[78] E. P. Day. I. Search for Diamagnetic Changes during Biomolecular Phase Transitions, II. Detection of Nuclear Magnetic Resonance using a Josephson Junction Magnetometer, and III. Information Content of Living Systems. PhD thesis, Stanford University, 1972.

[79] D. P. Osterman and S. J. Williamson, Rev. Sci. Instrum. 54, 1380 (1983).

[80] R. A. Webb, Rev. Sci. Instrum. 48, 1585 (1977). 
[81] K. Weyand, Rev. Sci. Instrum. 59, 971 (1088).

[82] Biomagnetic Technologies, Inc., 4174 Sorrento Valley Blvd., San Diego, CA 92121.

[83] Corning Inc., P.O. Box 5000, Corning; NY 14830.

[84] H. L. Anderson, Editor, A Physicist's Desk Reference (American Institute of Physics, New York, 1989).

[85] C. Connor. Low Frequency Magnetic Resonance with a dc SQUID. PhD thesis, University of California, Berkeley, 1989.

[86] Rapid Systems, Inc., 755 N. Northlake Way, Seattle, WA 98103.

[87] E. Fukushima and S. B. W. Roeder, Experimental Pulse NMR (AddisonWesley Publishing, Reading, 1981).

[88] N. N. Greenwood and A. Earnshaw, Chemistry of the Elements (Pergamon Press, Oxford, 1984).

[89] A. H. Silver, J. Chem. Phys. 32, 959 (1960).

[90] R. S. Pease, Acta Crystallogr. 5, 356 (1952).

[91] J. Thomas, Jr., N. E. Weston, and T. E. O'Connor, J. Am. Chem. Soc. 84, 4619 (1.963).

[92] A. H. Silver and P. J. Bray, J. Chem. Phys, 32, 288 (1960).

[93] Alpha Products, P.O. Box 8247, Ward Hill, MA 01835-0747.

[94] Electronic Space Products International, 5310-U Derry Ave., Agoura, CA 91301.

[95] F. A. Cotton and G. Wilkinson, Advanced Inorganic Chemistry, 4th edition (John Wiley and Sons, New York, 1980).

[96] C. Connor, private communication. Defence Research Establishment Pacific, CFB Esquimalt, FMO Victoria, B.C., VOS 1B0, Canada.

[97] A. H. Silver and P. J. Bray, J. Chem. Phys. 29, 984 (1958).

[98] P. J. Bray, J. O. Edwards, J. G. O'Keefe, V. F. Ross, and I. Tatsuzaki, J. Chem. Phys. 35, 435 (1961).

[99] G. E. Jellison, Jr., L. W. Panek, P. J. Bray, and G. B. Rouse, Jr., J. Chem. Phys. 66, 802 (1977).

[100] L. G. Butler and T. L. Brown, J. Magn. Reson. 42, 120 (1981).

[101] P. W. France and M. Wadsworth, J. Magn. Reson. 49, 48 (1982). 
[102] G. L. Turner, K. A. Smith, R. J. Kirkpatrick; and E. Oldfield, J. Magn. Reson. 67, 544 (1986).

[103] W. H. Zachariasen, Acta Crystallogr. 7, 305 (1954).

[104] B. M. Craven and T. M. Sabine, Acta Crystallogr, 20, 214 (1966).

[105] J.T. Baker Chemical Co., 222 Red School Lane, Phillipsburg, NJ 08865.

[106] P. H. Kemp, The Chemistry of Borates, Part I (Borax Consolidated Ltd., London, 1956).

[107] R. B. Creel, J. Magn. Reson. 50, 81 (1982).

[108] R. C. Weast, Editor, CRC Handbook of Chemistry and Physics, 62nd edition (CRC Press, Boca Raton, 1981).

[109] G. W. Leppelmeier and E. L. Hahn, Phys. Rev. 141, 724 (1966).

[110] W. H. Zachariasen, J. Am. Chem. Soc. 54, 8 (1932).

[111] P. J. Bray, Bull. Am. Phys. Soc. 34, 900 (1989).

[112] P. J. Bray, S. J. Gravina, and D. H. Lee, Bull. Am. Phys. Soc, 34, 954 (1989).

[113] P. J. Bray, S. J. Gravina, and D. H. Lee, Poster Presentation, Xth International Symposium on Nuclear Quadrupole Resonance Spectroscopy(Takayama, Japan, 1989).

[114] L. Allen and J. H. Eberly, Optical Resonance and Two-Level Atoms (Dover Publications, New York, 1987).

[115] P. J. Bray, S. J. Gravina, D. H. Hintenlang, and R. V. Mulkern, Magn. Reson. Rev. 13, 263 (1988).

[116] N. Ishizawa, T. Miyata, I. Minato, F. Marumo, and S. Iwai, Acta Crystallogr. B 36, 228 (1980).

[117] R. W. C. Wyckoff, The Structure of Crystals (Rheinhold, New York, 1935).

[118] R. W. C. Wyckoff, Crystal Structures (Interscience, New York, 1948).

[119] Union Carbide Corp., 9320 Chesapeake Drive, San Diego, CA 92123.

[120] R. J. Mahler, Phys. Rev. 188, 584 (1969).

[121] H. J. Jakobsen, J. Skibsted, H. Bildsøe, and N. Chr. Nielsen, J. Magn. Reson. 85, 173 (1989).

[122] C. M. Verber, H. P. Mahon, and W. H. Tantillar, Phys. Rev. 125, 1149 (1962).

[123] N. Bloembergen, Physica 15, 386 (1949). 
[124] A. H. Silver, T. Kushida, and J. Lambe, Phys, Rev, 125, 1147 (1962).

[125] T. Tagai, H. Reid, W. Joswig, and M. Korekawa, Z. Kristallogr. 160, 159 (1982).

[126] A. Samoson, private communication. Institute of Chemical Physics and Biophysics, Tallinn, Estonia.

[127] K. T. Mueller, G. C. Chingas, and A. Pines, Rev. Sci. Instrum. 62, 1445 (1991).

[128] G. M. Muha, J. Chem. Phys, 73, 4139 (1980).

[129] G. M. Muha, J. Magn, Reson. 49, 431 (1982).

[130] T. A. Scott, J. Chem. Phys. 36, 1459 (1962).

[131] J, R. Brookeman, M. M. McEnnan, and T. A. Scott, Phys. Rev. B 4, 3661 (1971).

[132] M. H. Cohen, Phys. Rev. 96, 1278 (1954).

[133] T. C. Wang, Phys. Rev. 99, 566 (1955).

[134] M. Bloom, L. Robinson, and G. M. Volkoff, Can. J. Phys. 36, 1286 (1958).

[135] R. Livingston and H. Zeldes, Tables of Eigenvalues for Pure Quadrupole Spectra, Spin 5/2, Oak Ridge National Laboratory Report ORNL-1913(1955).

[136] R. Bersohn, J. Chem. Phys. 20, 1505 (1952).

[137] V. S. Grechishkin and V. P. Anferov, in Advances in Nuclear Quadrupole Resonance, Vol. 4 (Heyden Press, London, 1980).

[138] L. I. Schiff, Quantum Mechanics, 3rd edition (McGraw-Hill, New York, 1968).

[139] W. H. Beyer, CRC Standard Mathematical Tables, 27th edition (CRC Press, Boca Raton, 1984).

[140] E. Kreyszig, Advanced Engineering Mathematics, 5th edition (John Wiley and Sons, New York, 1983). 

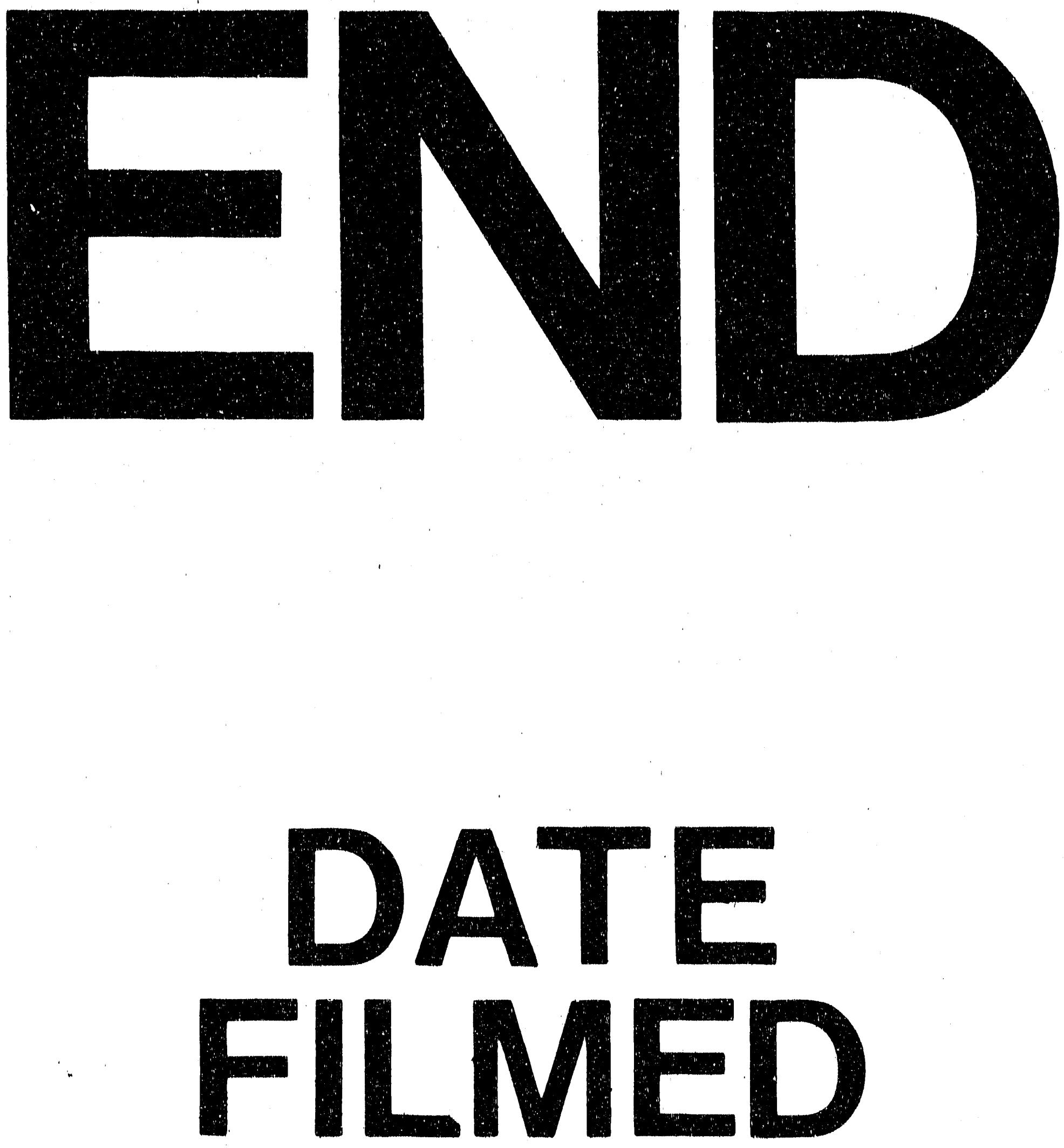

7

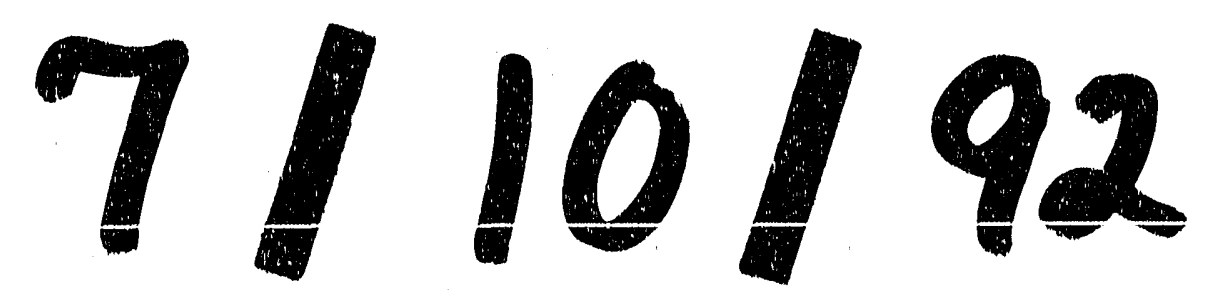


